\title{
Abstracts from the 1st International Conference on Alzheimer's disease and Related Neurodegenerative Disorders Wuhan, China
}




\section{Pathology and Pathophysiology}

1

Alzheimer Disease: Significance and Molecular Mechanism of Neurofibrillary Degeneration

Khalid Iqbal, Alejandra del C. Alonso, C.X. Gong, N. Haque, S. Khatoon, J.J. Pei, A. Sengupta, I. Tsujio, J.Z. Wang, Inge Grundke-Iqbal. New York State Institute for Basic Research, Staten Island, New York, USA

Neurofibrillary degeneration is pivotally involved in the pathogenesis of Alzheimer disease (AD) and several other tauopathies. In every single tauopathy known to date, the neurofibrillary changes are made up of abnormally hyperphosphorylated tau (P-tau). In AD brain P-tau is seen both as polymerized into tangles of paired helical filaments/straight filaments (PHF/SF) and as non-fibrillized cytosolic tau (AD P-tau). Unlike normal tau, both PHF-tau and AD P-tau are unable to promate in vitro assembly of tubulin into microtubules. Furthermore, the AD P-tau inhibits microtuble assembly and disassembles microtubules assembled with normal tau, MAP1 or MAP2. This inhibitory activity of AD P-tau involves sequestration of normal MAPs by the abnormal tau. The AD P-tau self-assembles in vitro into tangles of PHF and this self-assembly is inhibited by dephosphorylation and not deglycosylation. In vitro hyperphosphorylation ( $>10$ moles phosphate/mol tau) of recombinant human brain tau results in its self-assembly into tangles of PHF/SF. Activities of phosphoseryl/phosphothreonyl protein phosphatases (PP)-2A/PP-1 which regulate the phosphorylation of tau in brain are compromised in $\mathrm{AD}$ brain. Phosphorylation/dephosphorylation imbalance is one of the most promising therapeutic targets for $\mathrm{AD}$ and related tauopathies.

2

ER stress, Presenilins, and Alzheimer Pathogenesis

M. Takeda. Psychiatry \& Behavioral Proteomics, Department of PostGenomics, Osaka University Graduate School of Medicine, D3, 2-2 Yamadaoka, Suita City, Osaka, 565-0871, Japan

New protein is biosynthesized on ribosomes and folded into the proper tertiary structure in endoplasmic reticulum (ER) to fulfill biologically competent role. Genetic mutations, errors in transcription or translation, and protein denaturing by oxidative stress or heat shock stress all cause accumulation of unfolded proteins in ER lumen. Accumulation of unfolded proteins triggers the defense mechanism called unfolded protein response (UPR) in ER, which results in either degradation, suppressed transportation, or suppression of protein translation. When the amount of unfolded protein in ER exceeds the capacity of UPR, abnormal protein deposition may be accumulated within the cell. Since hypoperfusion, hypoxia, or hypoglycemia all can load ER stress to the brain in vivo, regulational mechanism of UPR may be closely related with pathophysiology of neuronal degeneration of Alzheimer's disease. Ire1p molecule has a sensor domain in lumenal side, kinase and RnaseL domain in cytoplasmic side. When unfolded protein is accumulated within the lumen of ER, Ire1p molecule is phosphorylated and dimerized on ER membrane. This conformational change of Ire $1 \mathrm{p}$ activates RNase $\mathrm{L}$ leading to splicing of Hac1 mRNA, which induces Haclp. Haclp acts as enhancer of chaperone genes such as GRP78 or GRP94, whose transcription is regulated under UPRE. Our data suggest the involvement of PS1 to ER-stress response, which may explain the neurodegenerative mechanism of FAD brain. Cells transfected with AD-linked PS1 mutation is shown to be more vulnerable to ER stress, and the vulnerability to ER-stress is explained by a decreased induction of GRP78, which is confirmed by the experiments demonstrating that the GRP78 chaperones rescue the neuronal death by ER-stress of cultured neurons with mutant PS1. Wild type PS1, as well as FAD-linked mutant PS1 binds to Ire $1 \mathrm{p}$, however, the mutant PS1 is unable to phosphorylate Ire1p, which results in suppressed induction of Haclp. There are three stress sensor molecules identified on ER membrane; Ire1p, ATF6, and PERK. ATF6 is shown to be cleaved under ER-stress and translocated to the nucleus to induce chaperone proteins. PERK phosphorylates eIF2, which causes suppression of protein translation after ER stress. PS1 is shown to be involved in the regulation of ER-stress induced UPR, the study of ER-stress is necessary to elucidate the mechanism of eurodegeneration.

Nicastrin, a component of presenilin complex is involved in $\beta$-/S3-cleavage of $\beta \mathbf{A P P} / N$ otch

F.S. Chen, G. Yu, M. Nishimura, S. Arawaka, A. Tandon, P.E. Fraser, P.G. Hyslop. Center for Research in Neurodegenerative Diseases, University of Toronto, Tanz Neuroscience Building, 6 Queen's Park Crescent West, Toronto, ON, M5S 3H2, Canada

The presenilin 1(PS1) and presenilin 2(PS2) are evolutionarily conserved, polytopic, transmembrane proteins that are components of separate high molec- ular weight complexes $(>250 \mathrm{kDa})$. Although the precise molecular mechanisms are unclear, PS1 and PS2 are important in an unusual form of proteolytic processing which involves cleavage through the transmembrane domains of $\beta$-amyloid precursor protein ( $\beta$ APP) and Notch. The former process (termed $\beta$-secretase cleavage) generates amyloid $\beta$-peptide $(\mathrm{A} \beta)$, which is involved in the pathogenesis of Alzheimer's disease (AD). The later (termed S3-site cleavage) generates Notch intracellular domain (NICD) and is involved in intercellular signaling. Some evidence has suggested that the presenilins may have direct catalytic activity. However, some additional observations argue that this activity likely requires interactions between the presenilins and other proteins within high molecular weight presenilin complexes. We have identified a novel component of the high molecular weight PS1 and PS2 complexes, nicastrin (NCT), a type 1 transmembrane glycoprotein. We have demonstrated that NCT interacts with both PS1 and PS2, binds to the substrates for $\beta$-secretase cleavage of $\beta \mathrm{APP}$ and to the substrate for S3-site cleavage of Notch, and is therefore involved in the production of $\mathrm{A} \beta$ and NICD. Missense mutations in a conserved hydrophilic domain of nicastrin increase $\mathrm{A} \beta$ secretion and NICD generation. Conversely, deletions in this domain inhibit $\mathrm{A} \beta$ and NICD production. However, although similar in direction, there were significant differences in the relative magnitude of their effects on NICD and $\mathrm{A} \beta$ secretion. These data suggest that nicastrin and presenilins are likely to be functional partners necessary for the intramembranous proteolytic processing of proteins such as Notch and $\beta$ APP. Exclusive Mediotemporal Pathology within the Spectrum of Frontotemporal Dementia

R. Ravid ${ }^{1}$, W. Kamphorst ${ }^{2}$, J.C. Van Swieten ${ }^{3}$, S. Rosso ${ }^{3}$, A. Holtrop, M. Kooreman, J Wouda ${ }^{1}$. ${ }^{1}$ Netherlands Brain Bank, Amsterdam, Netherlands, ${ }^{2}$ Free University Hospital, Amsterdam, Netherlands, ${ }^{3}$ Department of Neurology, Erasmus University Rotterdam, Netherlands

In this paper we present cases (Group 1) with the clinical diagnosis of frontotemporal dementia (FTD) and neuropathological changes which are restricted to the medio-temporal lobe. They were compared with cases (Group 2) who were clinically diagnosed as FTD ( 4 cases), (pre)senile Alzheimer disease ( 2 cases) and subcortical dementia ( 1 case) and neuropathologically resembled Pick C1 (Constantinidis). The presenting symptoms were family history of dementia, agitation, apathy, dysphasia and memory problems. In addition to conventional staining methods, antibodies against ubiquitin, AT8, Beta-A4 and GFAP were applied. In group 1 neuronal losses occurred in the hippocampus, parahippocampal gyrus, amygdala, and the entorhinal cortex bordering the amygdala. In group 2, the neuronal losses fitted the diagnosis of Pick $\mathrm{C} 1$. The Braak staging for tangles did not exceed stage 2 in both groups; the amyloid load according to Braak was AO in group $1, \mathrm{AO}, \mathrm{A}$ or B in group 2. The granular layer of the hippocampus in group 1 contained fine ubiquitin positive granules, in group 2 many spherical bodies resembling Pick bodies were seen and in ventral striatum, similar Pick-like bodies were present. Furthermore, predominantly in the second layer of the neocortex and in the striatal neuropil, ubiquitin positive splinters, threads and boomerangs occurred, which were entirely absent in group 1 . we report here the recognition of a new distinctive subgroup of patients within the spectrum of FTD with additional exclusive mediotemporal pathology.

Cellular Membrane Modification and Nicotinic Receptor Changes in the Pathogenesis of Alzheimer's Disease

Z.Z. Guan ${ }^{1,2}$, Y.G. Long ${ }^{2}$, X. Zhang ${ }^{1}$, M. Ousavi ${ }^{1}$, H. Unger ${ }^{1}$, G. Dallner ${ }^{3}$, A. Nordberg ${ }^{1}$. ${ }^{1}$ Department of NEUROTEC, Division of Molecular Neuropharmacology, Huddinge University Hospital, Karolinska Institute, S-141 86 Stockholm, Sweden; ${ }^{2}$ Department of Pathology, Guiyang Medical College, 550004 Guiyang, P.R. China; ${ }^{3}$ Department of Biochemistry and Biophyysics, Stockholm University, S-10691 Stockholm, Sweden

In postmortem brains of patients with $\mathrm{AD}$ and age-matched controls, the nAChR $\alpha 3, \alpha 4, \alpha 7$ and $\beta 2$ subunits were investigated by Western blot; all cellular membrane phospholipids and their ether subclasses were analyzed by high performance liquid chromatography; fatty acid compositions of these lipids were measured by gas chromatography. Phosphatidylethanolamine-Nmethyltransferase activity in $\mathrm{AD}$ brain was analysed by incorporation of the $\left[{ }^{3} \mathrm{H}\right]$-methyl group from $\left[{ }^{3} \mathrm{H}\right]-\mathrm{SAM}$ in to lipids. Lipid peroxidation was induced by free radical inducer and amyloid peptide (A $325-35)$ and $\mathrm{nAChR}$ expression was detected in PC 12 cells. The results showed that in AD patients, as compared to age-matched controls, the $\alpha 4, \alpha 3$ and $\alpha 7$ subunits were significantly reduced in some brain areas; decreased amount of phospholipids, modification of the lipid structure, and decreased activity of 
phosphatidylethanolamine-N-methyltransferase were observed in AD brain. Free radical insult $\left(\mathrm{FeSO}_{4}\right)$ induced dose-dependent increases in lipid peroxidation and toxicity to PC12 cells. Significant reductions in $\left[{ }^{3} \mathrm{H}\right]$ epibatidine and $\left[{ }^{125} \mathrm{I}\right] \alpha$-bungoratoxin binding sites, protein levels for the $\alpha 3$ and $\alpha 7$ subunits, and mRNA level for the $\alpha 7$ subunit were observed in the cells treated by $\mathrm{FeSO}_{4}$ compared to control. Exposure of PC12 cells to A $\beta$ induced lipid peroxidation and suppressed expression of nAChRs. However, pretreatment of cultural cells with antioxidant such as vitamin E prevented the inhibiting effect of $\mathrm{A} \beta$ on the nAChRs. These results reveal a decrease expression of $\mathrm{nAChRs}$ and changed cellular membrane structure in $\mathrm{AD}$ brain, and a reduction in numbers of $n A C h R s$ was resulted from lipid peroxidation and $A \beta$ in vitro. It is suggested that modification of cellular membrane and oxidative stress might be a mechanism involved in $\mathrm{nAChRs}$ deficit in $\mathrm{AD}$ brain.

Pathway of tau aggregation into alzheimer paired helical filaments

E. Mandelkow, M. von Bergen, A. Schneider, S. Barghorn, J. Biernat, E.M. Mandelkow. Max-Planck-Unit Struct. Mol. Biol., Hamburg, Germany

Alzheimer's disease and related tauopathies show the accumulation of tau protein into paired helical filaments (PHF) and the higher order structures of neurofibrillary tangles or neuropil threads. We are studying the pathway of aggregation in an effort to understand the molecular interactions and their determinants. (a) As a first step, the dimerization of tau by oxidation and formation of disulfide bridges greatly enhances aggregation so that tau dimer can be regarded as effective building blocks of PHFs. (b) Assembly is further enhanced by the presence of polyanionic cofactors (heparin, poly- Glu, RNA, or other acidic peptides). (c) Once these conditions are met, tau undergoes a nucleation phase involving oligomers of 4-7 tau dimers. The nucleation barrier can be overcome by external seeds such as PHFs from Alzheimer disease brains. (d) After nucleation, PHFs must elongate and later coalesce with other PHFs into tangles. Phosphorylation at key residues (e.g. Ser262, Ser214) detaches tau from microtubules, thus enhancing the pool of tau molecules. It was often assumed that phosphorylation also promotes the aggregation of tau into paired helical filaments. Our recent results show, however, that the opposite is the case. The same phosphorylation sites that are most potent in dissociating tau from microtubules are also most potent in inhibiting PHF assembly (e.g. S262, S214). The other sites (mostly in Ser- Pro motifs) show a similar tendency but much less pronounced. Once PHFs are formed, a fraction of the sites can still be phosphorylated in the polymer and are therefore accessible to kinases. Another unresolved question is how tau forms apparently regular fibers (the PHFs) with an "amyloid" character (in terms of dye binding such as thioflavin S), even though the tau molecule shows a mostly random coil structure in solution. This can be explained in part by the influence of a short motif, 306-VQIVYK-311 at the beginning of the third repeat, which tends to form beta structure and thus provides a basis for the self-assembly of the protein. Finally we have studied the effect of tau mutations observed in frontotemporal dementias (FTDP-17). Although they lie mostly in the microtubule-binding domain they have only a weak influence on tau-microtubule interactions. By contrast, they show an enhanced tendency for PHF aggregation in the presence of polyanions; this is particularly evident for the mutations P301L and delK280. Supported by Deutsche Forschungsgemeinschaft.

Tau protein and Alzheimer's disease: phosphorylation and role in intracellular traffic

E.M. Mandelkow, K. Stamer, R. Vogel, J. Biernat, E. Mandelkow. MaxPlanck-Unit for Struct Mol. Biol., Hamburg, Germany

One of the hallmarks of Alzheimer's disease is the aggregation and hyperphosphorylation of tau protein. We are studying the question of how the abnormal modifications are related to one another and how they affect the physiological functions of the neuron, such as axonal ransport. We have identified a number of phosphorylation sites, ncluding some that affect tau's interactions with microtubules (e.g. S214 during mitosis). Phosphorylation affects the tau-microtubule interaction to different extents. The most potent effect is observed with the kinase MARK (alias par-1) which phosphorylates Ser 262 of tau and similar KXGS motifs in MAP2 or MAP4. Overexpression of MARK in cells leads to hyperphosphorylation of MAPs on KXGS motifs and to the disassembly of microtubules, resulting in morphological changes and cell death [1]. On the other hand, the phosphorylation at the KXGS motifs in tau is important for tau-induced process formation in cell models [2]. This points to a role of MARK in the development and maintenance of cell polarity. In therefore studied the effect of tau on intracellular transport and found that tau inhibits the kinesin-dependent transport of vesicles and organelles in the plus-direction of microtubules [3]. As a result, minus-end directed transport by dynein predominates. We have now tracked single vesicles and organelles in live cells and find that tau does not change the speed of motors once attached to a microtubule, but increases the probability of slipping off the track (shorter run lengths) and decreases the probability of attachment [4]. The net effect is to cause clustering of mitochondria, peroxisomes, and intermediate filament protein near the cell center, retraction of the endoplasmatic reticulum, and slowdown of exocytosis. Furthermore we transfected rat primary cortex neurons with adenovirus-APP tagged with yellow fluorescent protein (YFP) and studied the anterograde and retrograde movement of APP-vesicles by video microscopy. When blue-fluorescent Tau (tagged with CFP) is co-transfected by an adenovirus vector, the transport of the APP-vesicles down the axon is inhibited, and APP-vesicles become clustered in the cell body. The data show a direct linkage between tau overexpression and APP trafficking which may be of significance in the pathogenesis of Alzheimer's disease. While tau inhibits organelle transport, the kinase MARK has the opposite effect. It facilitates organelle transport by removing tau from the microtubule tracks. This illustrates the interplay between motors, MAPs, and kinases in the local regulation of traffic. In the case of neuronal cells, mitochondria, peroxisomes, and intermediate filaments largely disappear from the cell processes, leading to energy deprivation and vulnerability of the cells. - Supported by DFG.

Redox-active transition metals are associated with non-base paired dna and rna in Azheimer disease

G. Perry $^{1}$, R.J. Castellani ${ }^{1}$, P.L.R. Harris ${ }^{1}$, S.L. Siedlak ${ }^{1}$, M.A. Taddeo $^{1}$, A.

Nunomura $^{2}$, H. Fujioka ${ }^{1}$, M.A. Smith ${ }^{1}$. ${ }^{1}$ Case Western Reserve University, Cleveland, Ohio, USA; ${ }^{2}$ Asahikawa Medical College, Asahikawa, Japan

Oxidative damage in Alzheimer disease (AD) is one of the earliest cytopathological abnormalities to affect neurons. To establish whether metalcatalyzed redox cycling was involved in such oxidative damage, we assessed sites of redox activity in tissue sections. While amyloid- $\beta$ deposits and neurofibrillary tangles are sites of catalytic redox activity detected by the in situ oxidation of 3,3'-diaminobenzidine, those neurons with the highest level of oxidative damage, and lacking pathological changes also contain potent sources of redox activity. This cytoplasmic redox activity was found in all cases of $\mathrm{AD}$, at lower levels in aged-matched controls and was virtually undetectable in younger controls. Importantly redox activity in these neurons, unlike the lesions was greatly reduced or abolished by treatment with $\mathrm{S} 1$ nuclease or RNase, or by chelation with deferoxamine. These data show that cytoplasmic redox activity in neurons is associated with RNase-sensitive sites in ribosomes and that RNA-mediated redox activity is dependent on chelatable transition metals. Dot blots of purified tRNA also demonstrated redox activity. These findings suggest that RNA, perhaps in conjunction with other factors, plays a integral role in the cytoplasmic redox activity characteristic of Alzheimer disease. Furthermore, the high level of oxidative damage to RNA in AD is likely directly related to bound transition metals that mediate $\mathrm{OH}$ radical attack.

Mechanism of inhibition of fibrillization of amyloid beta-protein by choline-containing zwitterionic lipids, and its stimulation by acidic phospholipids

A. Chauhan, V.PS. Chauhan, J. Wegiel. NYS Institute for Basic Research in Developmental Disabilities, 1050 Forest Hill Road, Staten Island, NY 10314, USA

Acidic lipids are oriented mainly at the cytoplasmic side of the membrane while zwitterionic lipids are located on the inner leaflet of the membrane. During peroxidation of membrane and apoptosis, acidic phospholipids are translocated from the inner to the outer leaflet of the membrane. Recently, we have reported that acidic phospholipids such as phosphatidic acid (PA), cardiolipin (CL), phosphatidylserine (PS) and phosphoinositides (PIs) interact with amyloid beta-protein ( $\mathrm{A} \beta 1-40$ and $\mathrm{A} \beta 1-42)$, and increase its fibrillization [Chauhan et al., Neurochem. Res. 25, 23-429 (2000)]. The fibrillization of $\mathrm{A} \beta$ is a nucleation-dependent phenomenon, which involves nucleation phase, followed by the growth of the nucleus. In this study, we have investigated the mechanism of effect of acidic phospholipids and zwitterionic lipids on $\mathrm{A} \beta$ fibril formation. The liposomes of phosphatidylcholine (PC) and PC/sphingomyelin (zwitterionic lipids present in the external bilayer) were found to decrease the aggregation of $\mathrm{A} \beta 1-40$ by increasing the nucleation time, and therefore, inhibiting the formation of $\mathrm{A} \beta$ nucleus in a concentration-dependent manner. These lipids also inhibited the growth rate of $\mathrm{A} \beta$ fibril formation when soluble $\mathrm{A} \beta 1-40$ was seeded with $\mathrm{A} \beta$ nuclei. Addition of $10 \%$ phosphatidic acid (PA) to the PC liposomes not only overcame the PC-induced changes in nucleation time but also increased the growth of the fibrils significantly. The inhibitory effect of PC on seeding of amyloid formation was also reversed by PA. Other acidic phospholipids such as CL, PS and PIs in mixed liposomes were also able to overcome the effect of $\mathrm{PC}$ in the order of PS $>$ PIs $>$ CL. These results suggest 
that choline-containing lipids such as $\mathrm{PC}$ and sphingomyelin, main constituents of external leaflet of bilayer, prevent $\mathrm{A} \beta$ from fibrillization by inhibiting both nucleation and growth phases of $\mathrm{A} \beta$ fibril formation. Externalization of acidic phospholipids (during peroxidation and apoptosis) in Alzheimer's disease (AD) onto the outer leaflet of bilayer, make these lipids accessible for interaction the $\mathrm{A} \beta$, and leads to increased $\mathrm{A} \beta$ fibrillization in $\mathrm{AD}$.

10

Tau phosphatases and neurofibrillary degeneration of Alzheimer disease C.X. Gong ${ }^{1}$, J.Z. Wang ${ }^{2}$, J.J. Pei ${ }^{3}$, I. Grundke-Iqbal ${ }^{1}$, K. Iqbal. ${ }^{1}$ New York State Institute for Basic Research in Developmental Disabilities, 1050 Forest Hill Road, Staten Island, New York 10314, USA; ${ }^{2}$ Department of Pathophysiology, Tongii Medical College, HUST, Wuhan 430030, P.R. China; ${ }^{3}$ Karolinska Institutet, Dept of Neurotec, Division of Geriatric Medicine, Huddinge Hospital, Sweden

The hyperphosphorylated form of microtubule-associated protein tau is the major protein component of paired helical filaments (PHF) of Alzheimer neurofibrillary tangles. The abnormal hyperphosphorylation of tau depresses its biological activity to promote microtubule assembly and appears to facilitate its biological activity to promote microtubule assembly and appears to facilitate its aggregation into PHF. The phosphorylation state of tau depends on the relative activites of tau protein kinases and phosphatases. Protein phosphatases (PP) are classically divided into four major types, i.e. PP1, PP2A, PP2B, and $\mathrm{PP} 2 \mathrm{C}$. We have systematically studied the phosphatases that may regulate tau phosphorylation. In vitro, $\mathrm{PP} 1, \mathrm{PP} 2 \mathrm{~A}$ and $\mathrm{PP} 2 \mathrm{~B}$, but not $\mathrm{PP} 2 \mathrm{C}$, were able to dephosphorylate tau at multiple phosphorylation sites. PP2A and PP2B also dephosphorylated PHF-tau at several sites. The dephosphorylation decreased the resistance of PHF-tau to proteolysis and restored tau's ability to promote the in vitro microtubule assembly. Treatment of Alzheimer neurofibrillary tangles with either PP2A or PP2B induced partial disassociation of the tangles and PHF structure. Immunohistochemically, all of the PP1, PP2A and PP2B were stained in neuronal cell bodies in postmortem human brain tissue sections, indicating the expression of these phosphatases in neurons. Although the protein expression of these phosphatases was found unchanged in brains with Alzheimer disease (AD), the activities of PP1 and PP2A towards a general substrate was found significantly decreased by $20 \%$ in AD neocortex. When assayed towards the hyperphosphorylated tau isolated from $\mathrm{AD}$ brain as a substrate, the tau phosphatase activity was found significantly decreased by $30 \%$ in AD brain as a compared with age-matched controls. In metabolically active rat brain slices, inhibition of PP2A alone by okadaic acid induced hyperphosphorylation of tau at many abnormal hyperphosphorylation sites and accumulation of tau in situ, both of which are features of AD pathology. The hyperphosphorylated tau isolated from the okadaic acid-treated brain slices had a reduced activity to bind to microtubules and to promote microtuble-assembly in vitro. However, selective inhibition of PP2B in the brain slices did not have any significant effect on tau phosphorylation, accumulation, or ability to bind and promote microtubules assembly. These studies suggest that PP2A is a major tau phosphatase that regulates the phosphorylation and processing of tau.

Abundant Short Chain L-3-Hydroxyacyl-CoA Dehydrogenase/ Type 10 $17 \beta$-Hydroxy- steroid Dehydrogenase in the Hippocampus of Mouse Alzheimer's Disease Model

X.Y. He, G.Y. Wen, G. Merz, D. Lin ${ }^{1}$, Y.Z. Yang, P. Mehta, S.Y. Yang. NYS Institute for Basic Research in Developmental Disabilities, Staten Island, New York 10314, USA, and ${ }^{1}$ Brookhaven National Laboratory, Upton, New York 11973, USA

A full-length cDNA encoding mouse brain short chain L-3-hydroxyacylCoA dehydrogenase (SCHAD)/type $1017 \beta$-Hydroxysteroid Dehydrogenase $(17 \beta$-HSD10) was cloned, and the amino acid sequence of this enzyme was deduced from the nucleotide sequence. Fusion of the first eleven residues of this enzyme to the N-terminus of GFP directed the reporter protein into mitochondria. Thus, the first 11 residues of mouse SCHAD/17 $\beta$-HSD10 was identified as a mitochondrial targeting signal that accounts for the intracellular localization of this enzyme. The deduced amino acid sequence enabled construction of a three-dimensional model, which revealed that if the primary structure of mouse SCHAD/17 $\beta$-HSD10 is truly as that reported by Powell et al. (J. Mol. Biol. 303, 311-327, 2000) the binding of coenzyme will be interfered by the indole of tryptophan-23. This model demonstrated that a glycine-rich region (G17XXXG21XG23) is essential to dehydrogenase activity. The last ten residues at the $\mathrm{C}$-terminal of this enzyme was used as an antigen to raise rabbit anti-SCHAD/17 $\beta$-HSD10 antibodies, by which an abundance of this enzyme in synaptic mitochondria of hippocampi of $\beta$ APP transgenic mice rather than normal controls was shown with the immunogold electron microscopy. High levels of this enzyme may disturb steroid hormone homeostasis in synapses and contribute to hippocampal plasticity of mouse Alzheimer's disease model.

12 Presenilin regulates function and processing of the cadherin family of proteins

N.K. Robakis. Department of Psychiatry and Fishberg Research Center for Neurobiology, Mount Sinai School of Medicine New York University, New York, NY 10029, USA

Presenilin-1 (PS1) is a polytopic transmembrane protein expressed in many tissues including embryonic and adult brain. PS1 mutations are responsible for most cases of early-onset autosomal dominant familial Alzheimer disease (FAD). PS1 facilitates processing of several typeI transmembrane receptors including the amyloid precursor protein (APP), Notch1 receptor and classic cadherin adhesion receptors. In addition, PS1 regulates production of $\mathrm{A} \beta$ peptide, promotes neuronal survival and regulates $\bullet$-secretase activity. Classic cadherins are a superfamily of type I transmembrane proteins including E(epithelial)-, N(neuronal)-, and VE(vascular)-cadherins. They mediate $\mathrm{Ca}^{2+}$-dependent cell-cell adhesion and signal transduction, and control critical events in cell fate, cell migration, and tissue development including neural, epithelial and endothelial tissues. The extracellular domain of cadherins effects homophilic interactions between cadherins on opposing cell surfaces. Cytoplasmic cadherins bind $\beta$ - or $\beta$-catenin which in turn binds $\beta$-catenin. The latter protein binds actin thus linking the cadherin/catenin adhesion complex to the cellular cytoskeleton. Cadherins are expressed in highly dynamic and specific patterns throughout embryonic and adult life of vertebrates. At least fifteen classic cadherins are expressed in the CNS. These proteins regulate neurogenesis, neurite outgrowth, synapse formation and synaptic plasticity. Recently we showed that PS1 concentrates at synaptic and epithelial cell-cell contact sites where it forms $\mathrm{Ca}^{++}$-dependent complexes with the cadherin/catenin adhesion system. PS1 binds directly to several classic cadherins including E-cadherin. This binding is mediated by the large cytoplasmic loop of PS1 and by cytoplasmic sequence 604-615 of mature E-cadherin. Furthermore, PS1 regulates the stability, function, and processing of the cadherin/catenin adhesion complexes. The cadherin sequences that bind PS1 play crucial roles in the function of neuronal, epithelial and endothelial cadherins. Thus, PS1 may regulate the function of multiple cadherin systems during development and adult life. That PS1 regulates function and processing of several transmembrane protein systems suggests that in addition to APP processing and $\mathrm{A} \beta$ production, PS1 FAD mutations may target additional protein systems including the cadherin family of proteins. Which of these potential targets is critical for development of AD remains unclear. There is however good evidence that $\mathrm{AD}$ is a heterogeneous disorder with multiple systems affected.

13

Calcium-channel activity of $\mathrm{A} \beta \mathrm{P}$ is responsible for the neuronal cell death S.K. Rhee, D.S. Kim. Department of Biochemistry, Yeungnam University, Kyongsan 712-749, South Korea

Amyloid- $\beta$-peptides (A $\beta$ Ps) reconstituted into phospholipid vesicles formed calcium-permeable and zinc-sensitive channels (Rhee et al., 1988, J. Biol. Chem. 273: 13379; Lin et al., 1999, Biochem. 38: 11189). In the present study we investigated whether the $\mathrm{A} \beta \mathrm{P}$-induced calcium channels affect the neuronal cell death. Immunofluorescence microscopic study showed that $\mathrm{A} \beta \mathrm{P}$ binds to the plasma membrane of IMR-32 cells (human neuronal cell-line), and that the binding was interfered by a polyclonal antibody raised against five amino acids (DAEFR) at N-terminal region of $\mathrm{A} \beta \mathrm{P}(1-42)$. MTT reduction assay was used to analyze the $\mathrm{A} \beta \mathrm{P}$-induced cytotoxicity. About $40 \%$ of IMR-32 cells incubated in the presence of $8(\mathrm{M} \mathrm{A} \beta \mathrm{P}(1-42)$ for 3 hrs was degenerated. However, the cytotoxicity of $\mathrm{A} \beta \mathrm{P}$ in IMR-32 was reduced by zinc, a calcium channel antagonist, while Trolox or DTT-treated cells remained unaffected. A real-time measurement of calcium concentration in IMR-32 using fura- 2 dye revealed that the addition of $\mathrm{A} \beta \mathrm{P}(1-42)$ elevated the intracellular calcium concentration within a few seconds. The intracellular calcium concentration was decreased upon adding zinc in a dose-dependent manner. Our results suggest that $\mathrm{A} \beta \mathrm{P}$ forms calcium-permeable, zinc-sensitive channels on the plasmas membrane of IMR-32 cells, and this channel-forming activity of $\mathrm{A} \beta \mathrm{P}(1-42)$ induces cell death. These data support the hypothesis (Hardy \& Higgins, 1992, Science 256:184) that the destruction of calcium homeostasis in neurons promotes the formation neurofibrillary tangles (NFT), which leads to Alzheiner's disease (AD). [This work was supported by grant No. 1999-1-205-005-3 from the Basic Research Program of the Korea Science \& Engineering Foundation]

14

Presenilin 1 regulates the $\alpha$-pathway of APP processing in the stable cell 
lines expressing aspartyl mutants of PS1 and PS1 deficiency fibroblasts X.Y. Sun, O. Murayama, S. Sato, H. Yamaguchi, A. Takashima. Lab for Alzheimer's Disease, BSI of the RIKEN, Wako, Japan

Generation of $\mathrm{A} \beta$ is derived from multiple enzymatic events of APP processing. Three major kinds of secretases are reported to be involved in the generation of $\mathrm{A} \beta, \alpha$-secretase cleaves $\mathrm{APP}$ within $\mathrm{A} \beta$ region, precluding $\mathrm{A} \beta$ formation. However, the activity of $\alpha$-secretase has been reported to regulate $\mathrm{A} \beta$ production. $\beta$-secretase is an aspartyl enzyme, which cleaves APP at the site of $\mathrm{A} \beta$ and 11 to generate a direct precursor of $\mathrm{A} \beta$ APPC99. Finally, $\gamma$-secretase cleaves $\mathrm{A} \beta$ ? at the $\mathrm{C}$-terminus of $\mathrm{A} \beta$ to release $\mathrm{A} \beta$. Increased evidence has emerged to point out that presenilin is significantly involved in the activity of $\gamma$-secretase. The observation that abnormal accumulation of multiple C-terminal fragments of APP occurs in both PS deficiency mice and cells overexpressing apartyl mutants of PS suggest that PS1 has multiple roles in APP processing. In this study, we utilized both the stable cell lines overexpressing apartyl mutants of PS1 and fibroblasts derived from PS1 deficiency mice to investigate APP processing. Quantification of $\alpha$ secreted APP and $\beta$ secreted APP from conditioned media showed that cells expressing aspartyl mutants of PS1 had a significant increase in sAPP-alph secretion and a slight increase in SAPP-beta. It is consistent with the findings that significant accumulations APPCTFalpha occurs in these cells. Cell surface labeling experiments reveled that surface sAPP was elevated. The results were also confirmed in PS1-deficiency fibroblasts. The mechanism underlying PS regulation of $\alpha$-pathway of APP processing has been investigated. Altogether, this study indicates that inactivation of PS1 function results in not only abolishment of $\gamma$-secretase activity but also alternation of APP processing.

15

Mitogen activated protein kinase (MAPK), MAPK kinase and P70S6 kinase are regulated by protein phosphatase $2 \mathrm{~A}$ in rat brain: Implication in neurofibrillary degeneration in Alzheimer's disease

J.J. Pei ${ }^{1}$, C.X. Gong ${ }^{2}$, W.L. An ${ }^{1}$, R.F. Cowburn ${ }^{1}$, B. Winblad ${ }^{1}$, I. Grundke$\mathrm{Iqbal}^{2}$, K. Iqbal ${ }^{2}$. ${ }^{1}$ Karolinska Institutet, Department of NEUROTEC, Division of Geriatric Medicine, Huddinge Hospital, Sweden; ${ }^{2}$ NYS Institute for Basic Research in Developmental Disabilities, 1050 Forest Hill Road, Staten Island, NY 10314, USA

The extracellular signal-regulated protein kinase (ERK1/2) of the mitogenactivated protein kinase (MAPK) family is thought to be involved in the formation of abnormally hyperphosphorylated tau in Alzheimer's disease (AD). Previously, we and others have shown that ERK1/2 and its upstream kinase, MEK, and downstream kinase, ribosomal P70S6 kinase, in activated form accumulate in neurons with neurofibrillary tangles (NFTs) in AD. Since the activities of these kinases are regulated by phosphorylation and the activity of protein phosphatase $2 \mathrm{~A}$ (PP2A) is compromised in $\mathrm{AD}$ brain, we investigated if a decrease in PP2A activity could underlie the activation of these kinases and the abnormal hyperphosphorylation of tau. Brain slices, 400 (m-thick, from adult rats, kept under metabolically active conditions in oxygenated (95\% $\mathrm{O}_{2}, 5 \% \mathrm{CO}_{2}$ ) artificial CSF were treated with $1.0 \mu \mathrm{M}$ okadaic acid $(\mathrm{OA})$ for 1 hour at $34^{\circ} \mathrm{C}$. Under this condition, PP2A activity was decreased to about $35 \%$ of the vehicle-treated control slices, and no inhibition of PP1 activity was detected. In the OA-treated brain slices, we observed a dramatic increase in the phosphorylation/activities of ERK1/2, MEK and P70S6 kinase both immunocytochemically and by Western blots using phosphorylation-dependent antibodies against these kinases. Hyperphosphorylation of tau at several abnormal hyperphosphorylation sites as seen in $\mathrm{AD}$ brain was also detected. OA-induced PP-2A inhibition preferentially activated the cytoplasmic MEK and P70S6 kinase. Treatment of $6 \mu \mathrm{m}$ sections of the OA-treated slices with purified PP-2A reversed the phosphorylation/activation of these kinases. These results suggest that PP-2A could down-regulate ERK1/2, MEK and P70S6 kinase through dephosphorylation at the serine/threonine sites of these kinases. Thus, in AD brain the decrease in PP-2A activity might cause the aberrant accumulation of activated ERK1/2, MEK and P70S6 kinase, and the abnormal hyperphosphorylation of tau both by an increase in its phosphorylation and a decrease in its dephosphorylation.

16

Mechanism of Alzheimer's disease?Amyloid Formation and Regulation H.X. Xu. The Rockefeller University, 1230 York Avenune, New York, NY 10021, USA

The study of the subcellular sites of $\beta$-amyloid ( $\mathrm{A} \beta$ formation is a central area of Alzheimer's Disease (AD) research. Using a well-established cellfree reconstituted system, we originally demonstrated that the predominant site of $\mathrm{A} \beta$ formation is the trans-Golgi network. We have also shown that the non-secreted and highly amyloidogenic $\mathrm{A} \beta-42$ peptides can be generated in the endoplasmic reticulum. While studying compounds that could alter
$\mathrm{A} \beta$ generation, we demonstrated that gonadal hormones, such as estrogen and testosterone, can reduce $\mathrm{A} \beta$ production, suggesting a mechanism through which hormone replacement therapy may be effective in preventing AD. In addition, recent clinical evidence indicates a role for insulin in AD. Thus, we have been investigating the effect of insulin in $\mathrm{A} \beta$ generation. Studies on the molecular events that coordinate the production of neuronal $\mathrm{A} \beta$ through signal transduction pathways, will be presented. Understanding the molecular mechanism of $\mathrm{A} \beta$ formation will be important for the development of effective therapies for $\mathrm{AD}$

17

How does $\mathbf{A} \beta$ deposition progress in the non-demented toward the Alzheimer stage?

H. Yamaguchi. Gunma University School of Health Sciences, Maebashi 3718514, Japan

Recently, longitudinal studies on non-demented elderly people revealed the presence of a stage, named mild cognitive impairment (MCI), between no cognitive impairment $(\mathrm{NCI})$ and mild $\mathrm{AD}$ stages. MCI refers to individuals who have a memory impairment greater than expected for their age, yet general cognitive function is preserved, and therefore, MCI subjects do not meet the criteria for AD. Neuropathological studies on MCI subjects revealed that they had an intermediate value of mean $\beta$-amyloid load between that in NCI and AD. MCI subjects had no or few neurofibrillary tangles (NFTs) in the association cortex, and corresponded to Braak stage II or III. From the results of studies on more than 100 non-demented elderly subjects, I would like to show the time sequence of pathological legions in the frontal association cortex in the pre-clinical stage of $\mathrm{AD}$ as follows: 1) Senile plaque density correlated well with the logarithmic data of insoluble $\mathrm{A} \beta$ measured by EIA. The amounts of A $\beta 42$-EIA increased dramatically in the late pre-clinical stage, whereas the A $\beta 42+$ plaque density increased in the early pre-clinical stage. 2) Neurofibrillary pathology appeared only in the areas with severe $\mathrm{A} \beta$ deposition, and in subjects aged over 70. 3) ApoE e4 allele enhanced the $\mathrm{A} \beta$ deposition in pre-senile subjects. 4) The morphology of cerebral $\mathrm{A} \beta$ deposition changed from diffuse plaques with small amounts of $\mathrm{A} \beta$ in each plaque in the early pre-clinical stage to primitive/neuritic plaques with larger amounts of $\mathrm{A} \beta$ in each plaque in the late pre-clinical stage. 5) $\mathrm{A} \beta$ depositon in diffuse plaques initiated on the cell surface plasma membrane as the membrane-bound form. 6) Some diffuse plaques may disappear, suggesting a dynamic balance between plaque formation and clearance.Our findings suggest that the prevention of $\mathrm{A} \beta$ deposition in the late pre-clinical stage can be a rational therapeutic target, especially in elderly people with ApoE e4 allele.

Role of a Brain Type $1017 \beta$-Hydroxysteroid Dehydrogenase in the Pathogenesis of Alzheimer's Disease

S.Y. Yang, X.Y. He, G. Merz, Y.Z. Yang, P. Mehta. NYS Institute for Basic Research in Developmental Disabilities, Staten Island, New York 10314, USA

Intracellular amyloid beta-peptide $(\mathrm{A} \beta)$ is generated in the endoplasmic reticulum. An $\mathrm{A} \beta$-binding protein, reportedly associated with the endoplasmic reticulum, has been designated as endoplasmic reticulum-associated $\mathrm{A} \beta$ binding protein (ERAB). ERAB reportedly mediates the neurotoxicity of $\mathrm{A} \beta$ because the overexpression of ERAB significantly increases $A \beta$-induced apoptosis. ERAB is actually encoded by the SCHAD gene mapped at chromosome Xp11.2, short chain L-3-hydroxyacyl-CoA dehydrogenase (SCHAD)/ERAB proved to be type $1017 \beta$-hydroxysteroid dehydrogenase (17 $\beta$-HSD10). Here we show that $17 \beta$-HSD10 (ERAB) is imported into mitochondria rather than being associated with the ER due to its mitochondrial targeting signals. Thus, it seems unlikely that $17 \beta$-HSD10 (ERAB) acts as a putative $\mathrm{A} \beta$ receptor at the ER as described previously. The roles of $17 \beta$-HSD10 in steroid hormone metabolism suggest that disturbance of steroid hormone homeostasis by high levels of brain $17 \beta$-HSD10, rather than the binding of $\mathrm{A} \beta$, underlies the involvement of this protein in Alzheimer's disease.

19

Phosphorylation Of Tau by Cdk5 and Gsk $3 \beta$ is Modulated by Aberrant Glycosylation at A Site-Specific Manner

F. Liu, T. Zaid, K. Iqbal, I. Grundke-Iqbal, C.X. Gong. Department of Neurochemistry, New York State Institute for Basic Research in Developmental Disabilities, Staten Island New York 10314, USA

Microtubule-associated Protein tau is abnormally hyperphosphorylated, glycosylated, and aggregated in affected neurons in Alzheimer's disease (AD). We have found that the Glycosylation precedes hrperphosphorylation of tau in $\mathrm{AD}$. In this study, we investigated the site-specific phosphorylation of the longest isoform of recombinant human tau, tau441, with cyclin dependent kinase 5 (CDK5) and glycogen synthase kinase- $3 \beta$ (GSK $3 \beta$ ), respectively. The Phosphorylation of specific sites was detected by employing phosphorylation- 
dependent and site specific tau antibodies. We found that CDK5 phosphorylated tau441 at Thr181, Ser199, Ser202, Thr205, Thr212, Ser214, Thr217, Thr231, Ser235, Ser396, and Thr404, but not at Ser262, Ser400, Thr403, Ser409, Ser413, or Ser422, GSK-3 $\beta$ phosphorylated all above CDK5- catalyzed sites except Ser235. The effectes of aberrant tau glycosylation on its subsequent phosphorylation by CDK5 and GSK- $3 \beta$ was studied by comparing the rate and extent of the phosphoylation of the glycosylated tau isolated from $\mathrm{AD}$ brain(AD-tau) before and after its deglycosylation by glycosidases. Deglycosylation of AD-tau depressed the subsequent phosphorylation with CDK5 at Thl81, Ser199, Ser202, Thr255, and Thr414, but not at Thr212; With GSK-3 $\beta$ at Thrl81, Ser202, Thr2r5, Ser217, and Thr404, but not at Ser199, Thr212, thr231, or Ser396. These data suggest that the aberrant glycosylation may modulate tau protein at a substrate level so that tau is easier to be phosphorylated at many abnormal hyper-phosphorylation sites as in AD.

20

Alzheimer-like hyperphosphrylation of tau in rat transfected with Cdk5 X.M. Liao, J.Z. Wang. Neuroscience institute, Pathophysiology Department, Tongji medical college HUST, Wuhan 430030, P.R. China

One major neuropathological feature of Alzheimer disease is neurofibrillary tangles (NFTs). NFTs are composed of hyper-phosphorylated tau protein. In affected neurons, the major mechanism of tau hyperphosphorylation is the imbalanced regulation of protein kinases and protein phosphatases. In vitro $\mathrm{Cdk} 5$ could phosphorlate tau at $\mathrm{AD}$-relevant epitopes. To investigate its effect on tau in vivo, we have wrapped the plasmid PcDNA3.1/cdk5 with liposome, then injected it into rat hippocampus bilaterally, thus induced the over-expression of cdk5 in vivo, caused an imbalance in phosphorylation and dephosphoralation system. We have measured the phosphorylation of tau by using phosphorylation dependent antibodies. It was found by immunohistochemistry and Western blotting that phosphorylation of tau at ser395/404 by PHF-1 was elevated in hippocampus at $12 \mathrm{~h}, 24 \mathrm{~h}$ and $48 \mathrm{~h}$ after injection and at the same time, the expression of cdk5 increased. It was resulted from these data that in cdk5 transfected rats the expression of cdk5 was elevated, and the over-expression of cdk5 in vivo induced Alzheimer-like hyperphosphorylation of tau.

21

Experimental study of the treatment of Parkinson's disease by transplantation of immortalized fibroblasts genetically modified by TH gene and GCH gene

S.D. Chen, X.W. Chen, Z.G. Liu, et al. Department of Neurology, Ruijin Hospital, Shanghai Second Medical University, Shanghai 200025, P.R. China

The purpose of this study is to explore the therapeutic efficacy of cotransplantation of immortalized fibroblasts genetically modified by tyrosine hydroxylase (TH) and GTP cyclohydrolase-1 $(\mathrm{GCH})$ genes in Parkinson's disease (PD), Eukaryotic plasmid vector encoding TH gene or GCH gene was constructed and introduced into the fibroblasts which had been immortalized by SV40 large antigen transformation. Immortalized fibroblasts which could stably express TH or GCH in vitro were co-transplanted into the striatum of 6-hydroxydopamine (6-OHDA)-induced hemiparkinsonian rats. The therapeutic efficacy of the transplantation was estimated by apomorphine-induced rotational behavior. TH gene expression was determined by TH immunohistochemistry and RT-PCR analyses. The long-term survival ability of the grafted cells was evaluated by fibronectin immunohistochemical staining. We observed the rotational behavior was significantly improved 2 weeks after grafting, and the behavioral recovery maintained for up to 14 weeks. The expression of exogenous TH gene paralleled the behavioral improvement. The transplanted cells survived for at least 38 weeks without formation of tumor or obvious immunological rejection. No TH-positive cells were noted in the grafted striatum at this time. Our results showed Immortalized fibroblasts transformed by SV40 large antigen possess safety and long-term survival ability when intracerebrally transplanted, and may be served as competitive target cells for ex vivo gene therapy for central nervous system disorders. Co-transplantation of immortalized fibroblasts genetically modified by TH and GCH gene may be developed as an potential method for the treatment of PD.

22

Neurotransmitter receptors and brain aging and Alzheimer's disease: Free radicals are promotional factor of brain aging

X. Cui, F. Wei, X. Zhang, et al. Institute of Gerontology and Geriatrics General Hospital,of People's Liberation Army, Beijing 100853, P.R. China

The following serie of reports aim at studying the relationship between changes of neurotransmitter receptors, brain aging (BA) and Alzheimer's Disease (AD). The first paper emphasizes on disclosing that FRs are initial factor of BA. Through rats, domestica flies and friut flies treated with D-galactose, we observed the simulational effect of BA and senescence. The results show:
(1) Oxygen stress effect. In the rats treated with D-galactose (50 mg.kg-1.d-1,6 w.S.C), the levels of Free Radicals were elevated. The SOD levels of rats heart, brain, liver were decreased, but the level of lipofuscin and MDA were elevated. It means that biochemical reaction of simulation aging are produced by oxidative stress in D-galactose rats. (2) Musca domestica and drosophila which intook D-galactose emerged promoting aging effect. The disposal of Gompertz formula shows that the life span manifesting, by the mortalily of $5 \%$, $50 \%$, and $95 \%$, were shorter than that of control group. Meantime, SOD level of musca domestica heads were decreased, MDA and lipofusin level were elevated. It shows that oxygen stress induced by D-galactose is involved in aging as an aging promotor. (3) The neurotransmitter receptors function take part in the effect of BA. Brain mRNA of rats treated with D-galactose were injected into Xenopus oocytes. The expressed transmitters receptor were inserted into oocytes membranes. Lender the action of agonists, we can record membrane currents of transmitter receptors activated by agonists. Their amplitudes show: the receptors currents induced by six antagonists (Ach, Glu, NMDA, 5-HT, DA, BA) of rats brain treated with D-galactose were lower than those of the control group. It suggests that oxygen stress induced by D-galactose acts on neurotransmitters receptors which involved in brain aging effects, being identical to receptor reaction of aging rats, which also indicated that oxygen stress is the reason of decrease of neurotransmitter receptors function. In conclusion, simulation of brain aging and senescence induced by D-galactose come from oxygen stress. Neurotransmitter receptors are involved in BA. FRs are dominant factor of decrease of receptors function.

The effects of Swedish mutation of APP gene on oxidative damage in the neuronal cells

S.M. Zhang ${ }^{1}$, C.Z. He ${ }^{1}$, M. Zhang ${ }^{1}$, X.Y. Sun ${ }^{2}$, et al. ${ }^{1}$ Department of Neurology, Tongji Hospital of Tong Medical College, Huazhong University of Science and Technology, Wuhan 430030, P.R. China; ${ }^{2}$ Institute of Physics and Chemistry, Japan

To study the effects of APP gene Swedish mutation on oxidative damage in the neuronal cells. Stably transfected B103 neuronal cells with the APPP695 cDNA containing the swedish double mutations $\left(\mathrm{APP}_{\mathrm{SW}}\right)$ and with the plasmid alone (Neo) were used. The cells were treated with different concentrations of $\mathrm{H}_{2} \mathrm{O}_{2}\left(10^{-6}-10^{-3} \mathrm{M}\right)$. MTT, LDH release, FCM and TUNEL were used to detect the degrees of oxidative damage induced by $\mathrm{H}_{2} \mathrm{O}_{2}$. It were showed that no significant differences existed in lower concentrations of $\mathrm{H}_{2} \mathrm{O}_{2}$ $\left(10^{-6}-10^{-5} \mathrm{M}\right)$ between two cell groups $(p>0.05)$, but the significant differences were noted in higher concentrations of $\mathrm{H}_{2} \mathrm{O}_{2}\left(10^{-5}-10^{-3} \mathrm{M}\right)$ $(p<0.05)$. It was also showed that the apoptotic index of B103(APP $\left.{ }_{\mathrm{SW}}\right)$ was higher than that of $\mathrm{B} 103(\mathrm{Neo})$ after treated by $500 \mu \mathrm{m} \mathrm{H}_{2} \mathrm{O}_{2}$. It was demonstrated that swedish type mutation of APP gene could improve the cellular susceptibility of neurons to damage and apoptosis induced by oxidative stress.

Distinct effects of green tea polyphenols on 6-hydroxydopamine-induced apoptosis in $\mathrm{PC12}$ cells

C.F. Jin, G.G. Nie, Y.L. Cao, et al. Institute of Biophysics, Academia, Beijing 100101, P.R. China

The purpose of this study is distinct effects green tea polyphenols on 6hydroxydopamine-induced apoptosis in PC12 cells.6-OHDA -induced apoptosis in PC12 cells were chose as the oxidative stress-induced model of PD in vitro. When the Pc12 cells were pretreated with five catechins respectively for 30 min before exposed to $250 \mu \mathrm{M}$ 6-OHDA, EGCG and ECG in 50-400 $\mu \mathrm{M}$ had obvious concentration- dependent protective effects on cell viability, EC and $(+) \mathrm{C}$ almost had no obvious effects, while the cell viability decreased in the case of EGC. Five catechins also showed and different effects on cell apoptotic morphology as measured with phase contrast microscope and fluorescence microscopy Only 200-400 $\mu \mathrm{M}$ EGCG and ECG kept PC12 cells adhering well. Pretreated with other catechins in any concentration, PC12 cells became round and some of them were detached. Moreover, as measured with fluorescence microscopy, flow cytometry and DNA fragment electrophoresis methods, 200-400 $\mu \mathrm{M}$ EGCG or ECG led to significant inhibition against typical apoptotic characteristics of PC12 cells, but others had little protective effects. There the classified protective effects of the five catechins were in the order: $\mathrm{ECG} \geqslant \mathrm{EGCG} \gg \mathrm{EC} \geqslant(+)-C>E G C$. These results indicated that EGCG and ECG might be potent neuroprotective agents for PD.

Azheimer-like phosphorylation of tau and neurofilament induced by cocaine in vivo

S.J. Liu, Z.Y. Fang, Y. Yang, X.C. Wang, et al. Neuroscience Institute, Patho- 
physiology Department, Tongji Medical College, HUST, Wuhan 430030, P.R. China

It was reported that injection of cocaine could lead into overexpression of Cyclin-dependent kinase 5 (CDK5) and p35 (activator of CDK5) in corpus striatum in rat brain. Furthermore, overactivition of CDK5, also named tau protein kinase II, was found in Alzheimer disease neuronal cell. To explore the relationship between cocaine induced CDK5 overexpression and Alzheimerlike hyperphosphorylation of tau and neurofilament, we injected peritoneal cocaine $(20 \mathrm{mg} / \mathrm{kg} / \mathrm{day})$ into rats and measured the phosphorylation of the above mentioned neuronal cytoskeletal proteins by using phosphorylation dependent antibodies. It was found by immunocytochemistry and Western blotting that phosphorylation of tau at Ser-199/202 determined by tau- 1 and Ser-395/404 by PHF-1, and nonphophor- neurofilament by SMI32 was elevated in hippocampus, cortex and striatum at day 8 and day 16 after the injection of the drug, when compared with saline control rat at the same regions. No significant difference was seen at day 4. Although cocaine injection could induce significantly Alzheimer-like hyperphosphorylation of neuronal cytoskeletal protein, the overexpression of CDK5 was not detected. It is suggested that peritoneal injection of cocaine induces Alzheimer-like hyperphosphorylation of tau and neurofilament in rat brain, but this effect is not relevant to an increase in overexpression of CDK5 protein.

26

Activation of Glycogen synthase kinase-3 induces in vivo Alzheimer-like hyperphosphorylation of cytoskeleton proteins and special memory impairment in rats

S.J. Liu, Z.Y. Fang, Y. Yang, X.C. Wang, et al. Neuroscience Institute, Pathophysiology Department, Tongji Medical College, HUST, Wuhan 430030, P.R. China

Accumulated data suggest that abnormal activation of glycogen synthase kinase-3 (GSK-3) is responsible for hyperphosphorylation of cytoskeletal proteins, such as tau and neurofilament, which are constitutive component of neurofibrillary tangles in Alzheimer disease. To investigate in vivo the role of GSK-3 in Alzheimer-like tau phosphorylation, we have injected lateral ventriclely wortmannin into rat brain, and determined by immunocytochemistry and Western blotting the correlation of tau phosphorylation and activation of GSK-3. We found that the level of Ser396/404 phosphorylated tau was significantly increased at $1 \mathrm{~h}$ and $6 \mathrm{~h}$, and was back to basal level at $12 \mathrm{~h}$ and $24 \mathrm{~h}$ after injection of $10 \mu \mathrm{mol} \cdot \mathrm{L}^{-1}$ wortmannin. As wortmannin activates GSK-3 indirectly through inhibition of PI3K, and the pathway between PI3K and GSK-3 involves inactivation of protein kinase $\mathrm{B}(\mathrm{PKB})$ and thus activation of protein kinase $\mathrm{C} \delta(\mathrm{PKC} \delta$ ). Activated $\mathrm{PKC} \delta$ may phosphorylate GSK-3 at Ser-9 (GSK-3 $\beta$ ) or at Ser-21 (GSK-3 $\alpha$ ) and thus inactivated the kinase. To stop this possible regulatory pathway and maintain a consistent activation of GSK-3, we injected at the same time both wortmannin and GF-109203X, a PKC $\delta$ inhibitor, into rat brain. The hyperphosphorylation of tau at Ser199/202 and Ser396/404 was prolonged to $24 \mathrm{~h}$ by injection of wortmannin plus different concentration of GF-109203X. The data obtained from Morris water maze study showed that an impaired spatial memory was induced at $24 \mathrm{~h}$ after injection of wortmannin plus GF-109203X but not wortmannin alone. To further confirm the relevance between activation of GSK-3 and Alzheimer-like tau phosphorylation and lesion in memory, we determined the level of activated GSK-3 by using phosphorylation and activity dependent antibody. The results showed that the level of Ser9 and Ser21 phosphorylated GSK-3 in hippocampus was significantly lower in wortmannin plus GF-109203X injected rats than that wortmannin alone or saline injected control. The data suggest that transient activation of GSK-3 via wortmannin induces transient hyperphosphorylation of tau at certain Alzheimer epitope, and prolonged activation of GSK-3 and tau hyperphosphorylation could only be achieved by simultaneous inhibition of PKC. Both GSK- $3 \alpha$ and GSK- $3 \beta$ were overactivated by injection of wortmannin plus GF-109203X, and a positive correlation present between overactivation of GSK-3 and tau hyperphosphorylation as well as memory impairment.

Neurotransmitter receptors and brain aging and Alzheimer's disease: The effect of a neuropeptide PIP on neuronal apoptosis caused by binary injure of $\mathrm{H}_{2} \mathrm{O}_{2}$ and amyloid \&szlig; protein

W.B. LI, B.L. Zhang, X. Cui, et al. Institute of Gerontology and Geriatrics General Hospital, of People's Liberation Army, Beijing 100853, P.R. China

Free radicals (FRs) and amyloid \&szlig; protein (A\&szlig;) have been generally accepted as malignant factor of Alzheimer's disease (AD) and brain aging (BA). They are the main reason of neuronal lose. The typical lesion of AD and BA is neuronal apoptosis. Up to date, almost all the experimental results about the research of neuronal apoptosis come from the single damage of FRs or A\&szlig;. Although this kind of results can provide detailed mechanism, the occurrence process in the body is the integral action of multiple factors. It is a compound injury caused by initial factor of brain aging-FRs and ADA\&szlig; and others. We haven't seen the research report of neuronal apoptosis caused by binary injury of $\mathrm{H}_{2} \mathrm{O}_{2}$ and A\&szlig. Our research is to observe the effect of PIP on apoptosis caused by this kind of binary injure. PIP is a neuropeptide which has neurotransmitter character and can invoke multiple effects. It can promote development, growth, differentiation, and nourish the nerves and prevent neuron from being damaged. PIP is a promising drug candidate which can counter BA and AD. Our results will favor the preventive drug research of BA and AD. The experimental results showed: (1) PIP can effectively counter the neuronal apoptosis caused by single injury of $\mathrm{H}_{2} \mathrm{O}_{2}$ and A\&szlig; or the binary injuris. It suggests that PIP can develop a control drug and be worth studying or exploring deeply. (2) PIP specific receptor antagonist can reverse the preventive effects of PIP. However, the action that PIP antagonist prohibited the neuronal apoptosis of A\&szlig;. But in tests that neuronal apoptosis induced by $\mathrm{H}_{2} \mathrm{O}_{2}$, the apoptosis protective of PIP can be not influenced by PIP receptor antagonist. It is indicated that the mechanism of neuronal apoptosis caused by $\mathrm{H}_{2} \mathrm{O}_{2}$ and A\&szlig; has different characters, being a new discovery worthy to study in future.

28

The Study of $\mathrm{H}_{2} \mathrm{O}_{2}$ Induced Apoptosis of $\mathrm{B} 103$ Cell Line Stably Transected with Swedish Mutation and the Metabolism of APP

M. Zhang, S.M. Zhang, C.Z. He, X.Y. Sun. Department of Neurology, Tongji Hospital, Tongji Medical College, Huazhong University of Science and Technique, Wuhan 430030, P.R. China

In order to observe the metabolism of APP under the condition of apoptosis and study the possible effects of oxidative stress and apoptosis in the pathogenesis of $\mathrm{AD}, 0.1 \mathrm{mM} \mathrm{H} \mathrm{H}_{2} \mathrm{O}_{2}$ was used to induce $\mathrm{B} 103$ cells stably transfected with Swedish mutation. After the inducement of $\mathrm{H}_{2} \mathrm{O}_{2}$, the typical features of apoptosis could be observed under the electron microscopy, the sub-G1 peak was shown in the flow cytometry and the rate of apoptosis was $12.91 \% \pm$ $3.05 \%$, obviously higher than that of the control $(P<0.01)$. In the western blot, $90 \sim 100 \mathrm{KDa}$ fragment could be seen before the inducement of $\mathrm{H}_{2} \mathrm{O}_{2}$. But after the inducement, we could only see $8 \mathrm{KDa}$ fragment in the film. So we consider that the oxidative stress can induce the apoptosis of B103 cells stably transfected with FAD mutation and the production of the toxic APP C terminal fragments. On one side the CTF can lead to the loss of the cells by accelerating the apoptosis through caspase III family, on the other hand the excision of CTF can negatively feedback the apoptosis too.

29

Im Amyloid Protein Precursor 63-73 Peptide Sequence Is Neurotrophic S.L. Sheng, R. Wang, F. Yang, et al. Beijing Brain Aging Research Laboratory, Xuan Wu Hospital, Capital University of Medical Sciences, Beijing, 100053 P.R. China

It is known that the 319-335 peptide sequence of amyloid precursor proin695(APP) is neurotrophic. A search was made to find other peptide sequences of the $\mathrm{N}$ terminus that were neurotrophic. 9 peptides of various lengths were made by chemical synthesis. Their actions on MTT metabolism in SY5Y neuroblastoma cells were studied. Two weeks after STZ had induced diabetes in mice and rats, APP63-73 was injected subcutaneously for 2 weeks at $0.2 \mu \mathrm{g} /$ time/day in mice and at $2 \mu \mathrm{g} /$ time/day in rats. Six weeks after rats were ovariectomized, APP63-73 at $2 \mu \mathrm{g} /$ time every other day was injected subcutaneously for 6 weeks. Ex vivo MTT testing showed that APP63-73 peptide was the shortest bioactive sequence, which could: 1 . Clearly increase cell surface area and length of axonal processes; 2 . Improve water maze performance of diabetic mice and rats, and of ovariectomized rats; 3 . Restore to normal the number of neurons immunoreactive for NGF and NF in diabetic mice, and neurons immunoreactive for estrogen receptor, alpha synuclein, AT-8 (monoclonal antibody against tau protein phosphorylated at 202/205 sites), pp-1, pp-2A, pp-2B, GSK3, and cdk5 in ovariectomized rats; 4 . Improve conduction speed along rat sciatic nerve. App63-73 had no effect on glucose, insulin and glucagon levels in blood. In conclusions, APP63-73 peptide can clearly improve neurodegeneration in experimental diabetic animals and ovariectomized rats. The mechanism by which APP63-73 peptide improves neurodegeneration due to insulin and estrogen deficiency remains to be studied.

30

Pairment of Signal Transduction Pathway for Neuronal Survival in Alzheimer's Disease

Y. Meng, Z.J. Ji and S.L. Sheng. Beijing Brain Aging Research Institute, Xuan Wu Hospital, Capital University of Medical Sciences, Beijing 100053, P.R. China

The aim of this study is to explore the mechanism of neuronal loss and 
apoptosis, which are primary characteristics in AD brain, through studying the expression of proteins related to signal transduction pathways important for neuronal survival. Hippocampal tissue was taken from AD and non-AD brains, three specimens from each category, and homogenized. Protein estimation was done by Lowry method. Equal amount of protein was taken from each specimen; immunoprecipitation was done and analyzed by Western blot; color development was done by alkaline phosphatase method. AD hippocampal tissue showed diminished expression of CREB, P-CREB, increased expression of apoptosis-related protein BAX, and diminished expression of apoptosisrelated protein $\mathrm{Bcl}-2$. In conclusions: The survival of mature neurons requires many neuron-survival factors, e.g. PI3K, CREB, P-CREB, Bcl-2 in AD hippocampus, indicating impairment of signal transduction factors important for maintaining neuronal survival in $\mathrm{AD}$, which could be a cause for neuronal apoptosis.

31

A Study of Screen for G-protein Rab3a and dUTPase that Interact with GIF by Yeast Two-hebrid System

H.W. Ren, Q.H. Kang, Q.L. Chen, B.G. Ru *. National laboratory of Protein Engineering, College of Life Science, Peking University, Beijing 100871, P.R. China

The purpose of this study was to elucidate the elusive fountional mechanism of GIF and the possible relationship between GIF and Alzheimer's disease. The yeast two-hybrid system was employed to screen for GIF-interacting protein(s). Bail plasmid pHyblex-GIF was constructed and used to screen $4.6 \times 106$ transformants of a matched human brain cDNA library. Twelve strong double positive colonies (lacZ $=$, His) were found by recotransforming with bait plasmid. After eliminate the false positives the putative positive colonies were sequenced and searched for homology in webs of NCBI (a NIH-founded Webpage).It was found that four library plasmids of which inserts encode fragments of known genes: Cytochrome C oxidase subunit II, mitochondrial ATP synthase subunit $\alpha$, nuclear dUTPase and protein Rab3a, respectively. Of the preceding four interaction protein fragments, Rab3b and nuclear dUTPase were based on an analysis, In order to test whether the fulllength Rab3a or dUTPase protein can interact with GIF, cDNA of Rab3a and nuclear dUTPase are amplified by PCR using total cDNAs from human fetal placenta as template, respectively. As shown by yeast two-hybrid system experiments, the full-length Rab3a specifically interacts with GIF, but not with MT-1, and the full-length nuclear dUTPase with two single amino acid replacement can't interact with GIF. In other hand, Rab3b cDNA also was subcloned into the prokaryotic fusion expression vector pGEX-4t-1 and conduct a primary research on the expression the purification of Rab3a protein in E.coli BL21. To verify the specific GIF-Rab3a interaction in other biochemical system ultimately, MT-1 and GIF are constructed in the hemagglutinin(HA)-tagging vector pSV-HA that can generate high level of HA-tagged MT-1 and GIF proteins in mammalian cell respectively. Rab3a is subcloned into anther epitope tagging vector p-Flag-CMV that can expression high level of Flag-tagged Rab3a ptotein in mammalian cell. Mammalian COS7 cells are cotransfected with the epitope-tagged bait and prey constructs. The final results from coimmunoprecipitation and Western blotting experiments confirm that Rab3a binds to GIF with specificity in a binding manner in COS7 cell, but not to MT-1, Rab3a plays an important role in the neurotransmitter release. It was discussed that the possible impacts of the specfic Rab3a-GIF interaction on neurotransmitter and Alzheimer's disease.

32

The protection of APP17-mer peptide against neuronal apoptosis induced by $\mathbf{A} \beta 25-35$

Z. Cai, L. Sheng. Beijing Research Laboratory for Brain Aging, Beijing Xuan-Wu Hospital, Beijing 100053, P.R. China

The purpose of this study is to study the neuroprotective effects of the APP17-mer peptide. Methods Cell apoptotic model was developed by using A $\beta 25$-35. Protective effects of APP17-mer peptide against A $\beta 25-35$ induced apoptosis in neuroblastoma cell line SH-SY5Y was investigated by using FCM assay and DNA Ladder assay. Moreover, relative Levels of Cellular peroxides and mitochondrial membrane potential were measured by using Confocal Laser scanning microscope, and the expression of AIF and NF-(B was detected by using wes tern blot. Results APP17-mer peptide Can decrease the apoptotic rate of the SH-SY5Y Cells exposed to $\mathrm{A} \beta_{25-35}(P<0.01)$, suppress oxyradical production $(P<0.05)$, preserve mitochondrial function, and upregulate the expression of NF- $\kappa \mathrm{B}$. Conclusion APP17-mer is protective against Cell apoptosis induced by $\mathrm{A} \beta 25-35$.

33

Alterations in Vascular Reactivity in Single- and Double-transgenic Mice co-expressing the Human APP-C100 and Mutant SOD1 Gene
S.R. Xue ${ }^{1}$, Q.X. $\mathrm{Li}^{2}$, C. Masters ${ }^{2}$, Z. Khalil ${ }^{3} .{ }^{1}$ Department of Neurology, the first affiliated hospital of SuZhou University, 96 Shizi street, SuZhou, P.R. China; ${ }^{2}$ Department of Pathology, University of Melbourne, Parkville, Victoria 3050, Australia; ${ }^{3}$ National Ageing Research Institute, University of Melbourne, Parkville, Victoria 3052, Australia

The purpose of this study is to explore the mechanism underlying changes of microvascular reactivity in single and double transgenic mice, we investigated the peripheral vascular reactivity to vascdilators acetylcholine and sodium niroprusside on perfused microvasculature of the hind footpads using nontransgenic, single transgenic mice expressing the human APP-C100 (TgC100.WT or $\mathrm{TgC} 10 \mathrm{~V} 717 \mathrm{~F}$ ) and double transgenic mice co-expressing human APP-C100 and human SOD1(G93A)gene. The results were that single $\mathrm{TgC1} 100$ and double Tg mice C100/SOD1(G93A) at 2-3 months old showed a statistical decrease of $28 \%$ in blood flux compared to nontransgenic control mice. In addition, the vasodilative responsiveness was markedly reduced to $34 \%$ in $8-9$ months old $\mathrm{TgC} 100$ mice compared to the control mice. On the other hand, there was no significant difference in the profile of vasodilative reaction between $\mathrm{TgC} 100$. WT and $\mathrm{TgC} 100 . \mathrm{V} 717 \mathrm{~F}$ mice. $\mathrm{TgC} 100$ and double $\mathrm{Tg}$ mice also contained higher levels of $\mathrm{Ab}$ peptide in plasma than that of nontransgenic mice $(p<0.01)$. The present study suggests that the altered reactivity of microvasculature may be mediated by circulating soluble $\mathrm{Ab}$ peptides. The mechanisms underlying vasoactivity of circulating $\mathrm{Ab}$ in $\mathrm{TgC} 100$ and $\mathrm{Tg}$ mice may involve endothelium and nonendothelium.

34

Toxic effects of beta-amyloid on neurons in the rat brain and the influence of glucocorticoid and age on it

H.H. Chen ${ }^{1}$, S.G. Sun ${ }^{2}$, J.Z. Wang ${ }^{3}$, et al. ${ }^{1}$ Department of Geriatrics, Wuhan Psychiatric Hospital. ${ }^{2}$ Department of Neurology, Union Hospital, Tongji Medical College, ${ }^{3}$ Department of Pathophysiology, Tongji Medical College, HuaZhong University of Science and Technoloy, Wuhan 430030, P.R. China

The aim of this study is to explore the relation between beta-amyloid and the pathogenesis of Alzheimer disease (AD). After beta-amyloid peptide was injected into the rats brain. we investigated the survey of the apoptosis in the brain of adult and aged rats by using TUNEL method and detected the content of acetylcholine in the rats hippocampi with the method of base-hydroxylamine color-contrast, then the influence of the factors of age and glucocorticoid to the toxic effect of beta-amyloid was analysed. The results show that Betaamyloid peptide may strongly induce the apoptosis of neurons in hippocampi, striatum and cortex $(p<0.05$ or $p<0.01)$ and lower the content of acetylcholine in hippocampi $(p<0.05$ or $p<0.01)$.In addition, the senility and glucocorticoid pretreatment may enhance the toxic effect of beta-amyloid $(p<0.05$ or $p<0.01$ ). In conclusion, Beta-amyloid may play a key role in the pathogenesis of Alzheimer disease through its induction on apoptosis of neurons and decreasing of the content of the acetylcholine. Effects of extracts of ginkgo biloba leaves on learning and memory disor-

S.G. Sun ${ }^{1}$, H.H. Chen ${ }^{2}$, C.Q. Liu ${ }^{1}$, A.Q. Liu ${ }^{2}$, E.T. Tong ${ }^{1} .{ }^{1}$ Department of Neurology, Union Hospital, Tongji Medical University, ${ }^{2}$ Department of Geriatrics, Wuhan Psychiatric Hospital. Wuhan 430022, P.R. China

The objective is to study the effects of extracts of ginkgo biloba leaves (EGB) on the higher nervous activities of central nervous system, and provide the experimental evidence for the therapeutic possibilities of EGB. Establishing the aged animal model with disorders of learning and memory abilities by applying Scopolamine and emploring learning-behavioral training (Y-maze test and avoiding darkness test) and biochemistry determination,We observed the changes of learning and memory- behavior and the contents of acetylcholine and protein in the hippocampus. The results showed EGB $(75,150$, $500 \mathrm{mg} \cdot \mathrm{kg}^{-1}$ ) obviously prevented the learning and memory dysfunction of the animal model in a dose-dependent style $(p<0.05$ or $p<0.01)$. The content of acetylcholine in hippocampus were obviously increased by EGB in the animal model $(p<0.05$ or $p<0.01)$. The results indicate EGB had effects on improvement of learning and memory dysfunction in dementia aged rats.

36

Effect and Mechanism of Fastigial Nucleus Stimulation on Vascular Dementia in Aging Rats

W.H. Fan, K.N. Chen. Department of Neurology, First Hospital, the Third Military Medical University, Chongqing 400038, P.R. China

The objective of this study is to investigate the effect and mechanism of fastigial nucleus stimulation (FNS) treating vascular dementia in aging 
rats. Chronic frontal cerebral hypoperfusion was performed in aging rats by permanent bilateral common carotid artery occlusion (PBCCAO) method, thus the animal vascular dementia (VD) model was established, The cognitive function of rats was measured in a computerized shuttle-training case before and after FNS, the brain tissues were studied by immunohistochemical method for cholinergic acetyltrans- ferase (ChAT) as a marker. The electrodes were implanted into bilateral cerebellar fastigial nucleus. Rectangular pules with intensity of current 70-75 $\mu \mathrm{A}$, frequency $50 \mathrm{~Hz}$, duration $0.5 \mathrm{~ms}$ were adapted for FNS. FNS persisting for $10 \mathrm{~d}$ was given once a day as the therapy group. The cognitive function of rats was obviously reduced after two months of the chronic cerebral hypoperfusion and became worse four months of that hypoperfusion. FNS could distinctly improve the cognitive function and evidently reduced the cholinergic nevronal cell loss in hippocampus and neocortex of VD in aging rats. These results suggest that FNS can greatly improve the cognitive function of vascular dementia in aging rats and FNS may be benefit to the prevetion of the impaired cholinergic neurons in the neocortex and hippocampus of VD rats.

37

\section{CR2 in postmortem Parkinson's disease brain tissue}

Y.L. Liu, X.J. Zhang. Department of Neurology, Second Hspitol of Hebei Medical University, Shijiazhuang, P.R. China

The aim of this study was to investigate the distribution of CR2 in brain sections from cases of PD and some other neurological disorders, as well as from non-neurological controls. Blocks of brain tissues were dissected at autopsy from the hippocampus and temporal isocortex. Cases explored in this study included one Parkson's disease (PD) patient and one familial PD case, three multiple sclerosis cases, 2 dying of cardiovascular disease. The results found that some lymphocytes, presumably B-cell, and follicular dendritic cells (FDC) in spleen and tonsil tissues were intensely stained by both CR 2 antibodies. the antibody to CR2 also stained occasional round cells in both control and pathological brain tissues, again presumable B-lymphocytes. Erythrocytes in the vasculature, as well as in incidentally found small parenchyma of hemorrhages were negative. In PD brain, some neurofibrillary tangles were weakly stained at high power.Astrocytes could be seen in sections of MS brain, with the astrocytes at inner edge of the plaques in multiple sclerosis being staining more intensely. The result indicated that PD is one of the neurodegenerative disease which inflammation may play some role in the development of the disease.

38

Proliferation, Migration and Differentiationof Neural Progenitor Cells Following Mechanical Injury of Fimbria-Fornix

J.N. Tong ${ }^{1}$, Y.W. Ruan ${ }^{2}$, Z.B. Yao ${ }^{2}$. ${ }^{1}$ Department of Neurology, Central Hospital of Jinzhou, 121000 P.R. China; ${ }^{2}$ Department of Anatomy, Sun Yatsen University of Medical Sciences, Guangzhou 510089, P.R. China

The objective is to explore proliferation, migration and differentiation of neural progenitor cells following mechanical injury of Fimbria-Fornix. The Fimbria-Fornix of adult SD rats were cut transversely in a stereotaxic apparatus and the rats were injected intraperitoneally three times with $\mathrm{BrdU}(50 \mathrm{mg} / \mathrm{kg})$ at $1,3,5,7,10,15,20$ and $30 \mathrm{~d}$ after injury and sacrificed after the last injection. The change of proliferation, migration and differentiation of neural progenitor cells after brain damage was observed by BrdU, Nestin, GFAP and NSE single or double immunostaining. The results showed that at $3 \mathrm{~d}$ after injury, BrdU-positive cells began to increase in the SVZ. At $5 \mathrm{~d}$ after injury, BrdU-positive cells increased considerably in the medial SVZ. At 10d after injury, the increase in BrdU-positive cells was significant in the dorsolateral $\mathrm{SVZ}$. From $10 \mathrm{~d}$ after injury on, BrdU-positive cells began to migrate to the area of lesion and arrived at $20 \mathrm{~d}$ after injury. Double-label immunostaining show that these migratory BrdU-positive cells were Nestin-positive. In conclusion, The mechanical damage of Fimbria-Fornix can induced proliferation of neural progenitor cells which can migrate into the area of lesion probably in order to participate the repair of brain damage.

39

Calcium Involved in PC12 Cells Apoptosis Induced by $\beta$-Amyloid Protein M. Shao, S.D. Chen, Z.G. Liu. Department of Neurology, Ruijin Hospital, Shanghai Alzheimer Disease Center, Shanghai Second Medical University, Shanghai 200025, P.R. China

The objective of this study is to study the effects of soluble $\mathrm{A} \beta_{25}-35$ on intracellular calcium and enhancement of calcium induced by potassium chloride, PC12 cells viability was detected by MTT assay and intracellular calcium change was observed by laser confocal microscope. Our results showed cell viability induced by $10 \mu \mathrm{M} \mathrm{A} \beta_{25}-35$, pretreatment of $5 \mu \mathrm{M}$ nifedipine and $10 \mu \mathrm{M}$ nifedipine was $65.5 \pm 7.6 \%, 74.89 \pm 3 \%$ and $89.03 \pm 2.4 \%$
( $\mathrm{n}=5 \%$ control). There was significant difference between $10 \mu \mathrm{M} \mathrm{A} \beta_{25-35}$ group and $\mathrm{A} \beta_{25-35}$ group $(p<0.01)$. The degree of intracellular calcium enhancement showed $\mathrm{A} \beta_{25-35}$ dose-response manner. With $0.1 \mu \mathrm{M}$, $1 \mu \mathrm{M}, 10 \mu \mathrm{M}, 20 \mu \mathrm{M}, 30 \mu \mathrm{M} \mathrm{A} \beta_{25}-35$, the degree of enhancement was $6.38 \pm 3.3 \%(n=16), 6.42 \pm 1.8 \%(n=19), 62.2 \pm 1.1 \%(n=21)$, $69.3+10.5 \%(n=19)$ and $107.5+11.1 \%(n=33)$ respectively. Reaction to the elevation of $\mathrm{A} \beta_{25}-35$ was found in $60 \%$ of PC12 cells. Kcl could induce a rapid-initiated and rapid-recovered calcium enhancement, and pretreatment of $\mathrm{A} \beta_{25}-35$ could amplify the reaction of $\mathrm{KCl}$ to intracellular calcium. These reactions disappeared after addition of calcium free Hank's solution and could be antagonized by nifedipine through $\mathrm{L}$-voltage gated calcium channel mechanism. Our results suggest that the calcium-enhancement effects of $\mathrm{A} \beta_{25}-35$ were dependent on extracellular calcium and L-VOCC on cellular membrane. $\mathrm{A} \beta_{25}-35$ could make PC12 cells more venerable to physiological factors.

Study on the PC12 cell Apoptosis induced by Beta-amyloid Protein

H.D. Tang, M. Shao, Z.F. Weng, et al. Department of Neurology, Ruijin Hospital, Shanghai Second Medical University, Shanghai 200025, P.R. China The purpose of this study is to investigate the mechanism of PC12 cell death induced by $\mathrm{A} \beta_{25}-35$, MTT assay was used to analyze the effect of $\mathrm{A} \beta_{25}-35$ on cell viability; Electron microscope and DNA electrophoresis were applied to measure the type of PC12 cell damage; and immunohistochemistry and Western blot were utilized to determine whether $\mathrm{p} 53$ protein was involved in the cell damage. MTT assay showed that cell viability decreased with the increasing concentration of $\mathrm{A} \beta_{25}-35$. Electron microscope and DNA electrophoresis proved the cell apoptosis occurred after $\mathrm{A} \beta_{25}-35$ treatment. Immunohistochemistry and Western blot demonstrated the positive expression of $\mathrm{p} 53$ protein in $\mathrm{A} \beta_{25}-35$-treatment, but not in naive-treatment. Our results show $\mathrm{A} \beta_{25}-35$ can induce PC12 cell apoptosis, and $\mathrm{p} 53$ protein is involved in this procedure.

41

Effect of Nicotinamide on Expression of Bax Protein and Neural Apoptosis in Substantia Nigra of Mice with Parkinson's disease

D.Y. Wang ${ }^{1}$, C.D. Zhang ${ }^{2}$. ${ }^{1}$ Department of Neurology, Central Hospital of Jinzhou, 121000 P.R. China; ${ }^{2}$ Department of Neurology, First Affiliated Hospital of P.R. China Medical University, Shenyang 110001, P.R. China

The purpose is to investigate the possible role of apoptosis in the pathogenesis of Parkinson's disease(PD) and the antiapoptotic effect of nicotinamide on nigral neurons of PD mice. C57BL mice were intraperitoneally treated with 1-methy 1-4-phenyl -1,2,3,6- tetrahydropyridine (MPTP). MPTP group received MPTP at a dose of $30 \mathrm{mg} / \mathrm{kg}$ for 5 days. Nicotinamide+MPTP group received nicotinamide at a dose of $500 \mathrm{mg} / \mathrm{kg}$ every time before MPTP used. Then TUNEL and Bax stains of neuronal apoptosis in substantia nigra (SNc) were performed on all mice. The results showed that Nicotinamide+MPTP group was with a lower Bax protein expression in the SNc cytoplasms compared with MPTP group (the average gray scale value of Bax positive cells was $107.59 \pm 2.50$ and $80.59 \pm 3.73, p<0.05)$. The TUNEL positive nuclei in the SNc of MPTP group were much more than nicotinamide+MPTP group's (the quantity of TUNEL positive nuclei was $19.43 \pm 3.33$ and $6.33 \pm 2.35$, $p<0.05$ ).In conclusion, this study indicated that nicotinamide could decrease Bax protein expression induced by MPTP and decrease neural apoptosis in SNc of PD mice.

42

Neurotransmitter receptors and brain aging and Alzheimer's disease: The mechanism of neurotransmitter receptors damage caused by free radicals in brain aging

F.N. Huang, W.P. Yang, J.G. Zhang, et al. Institute of Gerontology and Geriatrics General Hospital, of People's Liberation Army, Beijing 100853, P.R. China

The paper tries to demonstrate two questions: (1) Free radicals (FRs) directly affect neurotransmitter receptors, cause injury of electrical activities and inhibit their function. (2) How FRs affect neurotransmitter receptors expression and what the pathogene is. We adopt Oxenopus oocytes and observed: 1. FRs can affect expressed receptor protein, lower of electrical activities and damage receptors function. When the expressed transmitter receptor of adult rats brain mRNA were treated with hypoxanthine and xanthine oxidase reaction system, superoxide anion free radicals (SAFRs) were produced. Membrane potential induced by agonists Ach, Glu and 5-Ht were lower than those in control group. It suggests that SAFRs can act on expressed receptor molecules, lower the potential and result in the decrease of receptor function. 2. BA was promoted by FRs, which affect the expression of neurotransmitter receptors. After the 
expressed receptors of brain mRNA of D-galactosemic rats were activated by agonists, Ach, Glu, NMDA, 5-HT DA and GABA, the membrane currents were lower than those of electrical activities of adults. Because the expressed brain receptor currents were lower than that of control, it is reasonable to estimate that treatment with D-galactose can interfere with the transcription or its temples. Their expressed receptor currents are lower than those in control group, proving that anterior transicription stage of expression of transmitter receptor gene are implicated in D-galactosemic rats. 3. The amplitudes of neurotransmitter receptors in aged brain were lower than that of adult control group. When we injected aged rats (30 months) mRNA into oocytes, membrane currents of expressed receptors were lower than those in control group (under the activation of Ach, Glu, 5-HT and so on). As seen in brain mRNA treated with D-galactose it is showed that the stage of anterior transcription levels were lower than those of control. The result proves that anterior tracription stage of transmitter receptor expression is damaged by oxygen stress in aged rats.In conclusion, FRs can affect every stages of transmitter receptors genes expression and present the function decrease of post-translational receptor protein, reflecting the decreased currents of transmitter receptors. All these results demonstrate that receptors function are injured by oxygen stress with variant mechanism.

43

Effect of Electroacupuncture on Content of Glutamic Acid in the Bilateral Striatum in rat with Parkinson's disease

J. Ma, Ts. Tian, G.J. Sun. Hubei College of Traditional Chinese Medicine, Wuhan 430061, P.R. Chin

The objective is to study the change of the content of glumate in the bilateral striatal in rat with Parkinson's disease and the effect of electroacupuncture on the change. Adopting Parkinson's mouse model induced by 6-hydroxy-dopa, the rats were divided into four groups: normal group, moot operation group in which physiological saline was used, model group and electroacupuncture group in which the point Fengfu and the point Taicong were needled. The contents of Glu in bilateral striatum of every group were detected. The content of Glu in right (destractive side) striatum is significantly higher than the normal group $(p<0.05)$ and the opposite side $(p<0.05)$. Electrotherapy can obviously decrease the content of Glu in right striatum in PD mouse $(p<$ $0.05)$. Glutamic acid takes an important role in the accurance of Parkinson disease. To decrease the content of Glu in striatum is one effective approach of electrotherapy treating Parkinson's disease.

44

The Effect of APP 17-mer Peptide on the Neurotoxicity of A $\beta$

R. Wang, J.Y. Zhang, Z.J. Ji, et al. Beijing Aging Research Laboratory, Xuan Wu Hospital, Capital University of Medical Sciences, Beijing 100053, P.R. China

The present work aims at adducing further proof of the neurotrophic effect of $\beta$-amyloid(A $\beta$ ) precursor protein(APP) 319-335(APP17-mer peptide), through studying the effect of APP17-mer peptide on the neurotoxicity of A $\beta$, thereby providing scientific evidence for the pathogenesis of $A D$ and its treatment. APP 17-mer peptide and $\mathrm{A} \beta_{25}-35$ was synthesized by solid phase method and purified by HPLC. Human neuroblastoma cells SY5Y were segregated into 3 groups: normal control group; $\mathrm{A} \beta_{25-35}(10 \mu \mathrm{mol} / \mathrm{L})$ damaged Group; and $\mathrm{A} \beta_{25}-35(10 \mu \mathrm{mol} / \mathrm{L})+\mathrm{APP} 17-\mathrm{mer}$ peptide $(10(\mathrm{~mol} / \mathrm{L})$ for neuroprotection group. Cell count, MTT metabolic rate, LDH leakage rate, axonal length, area of cell body, NT-3 immunohistorychemical staining and concentration of cytosolic calcium $\left(\mathrm{Ca}^{2+}\right)$ were used as indicators. Compare with normal control group, $\mathrm{A} \beta_{25}-35$ damaged group showed reduced cell count and MTT metabolic rate, increased LDH leakage rate, diminished axonal length and area of cell body, reduced NT-3 expression, increased concentration of cytosolic calcium $\left(\mathrm{Ca}^{2+}\right)$, but the addition of APP17-mer peptide normalized the foregoing changes. In conclusions, APP17-mer peptide is neurotrophic. It can diminished neurotoxicity of $\mathrm{A} \beta$.

45

Detection of mRNA Level and Mutation of Neurofilament in Alzheimer's disease

Y.P. Wang, X.G. Liu, Q. Wang, J.Z. Wang. Department of Pathophysiology, Tongji Medical College, Huazhong University of Science and Technology, Wuhan 430030, P.R. China.

Cytoskeletal disruption is a key pathological change in numerous human neurodegenerative diseases including Alzheimer's disease and amyotrophic lateral sclerosis (ALS). Neurofilament proteins (NF) are major neuronal cytoskeletal components. It was reported that abnormal neurofilament protein distribution and phosphorylation contributes to the cytoskeletal pathology of Alzheimer's disease. Our precious studies also showed that the level of neu- rofilament protein subunits in AD brain was elevated. To explore the possible mechanism that led to this elevation, we first measured in the present study the mRNA level of corresponding neurofilament subunits by using semiquantitative reverse transcription PCR (RT-PCR) technique. It was found that the level of NF-M and NF-L were significantly in AD $(n=8)$ than Huntington disease control $(n=7)$. AS neurofilament gene mutation was reportedly present in some other neurodegenerative disease, such as in sporadic ALS patients, we also detected this possibility in $\mathrm{AD}$ samples used in the present study by Single strand DNA conformation polymorphism (SSCP) technique. We found one mutation in neurofilament-M gene. Our results suggest that the enhancement of transcription is not the reason for the increase of content of neurofilament; neurofilament mutation may be involved in the pathogenesis for some $\mathrm{AD}$ patients.

46

The Effect of Amyloid Precursor Protein on the Differentiation of Neuroblastma Cell

Y.P. Wang, Z.F. Wang, J. Yu, Y.C. Zhang, J.Z. Wang. Department of Pathophysiology, Tongji Medical College, Huazhong University of Science and Technology, Wuhan 430030, P.R. China.

The main component of Alzheimer's disease (AD) senile plaques is amyloid- $\beta$ peptide $(\mathrm{A} \beta)$, a proteolytic fragment of the amyloid precursor protein (APP). APP mutations, which are causative of familial Alzheimer's disease (FAD), alter the processing of APP and lead to excess production and extra-cellular deposition of A $\beta$ peptide. The physiological role of APP and $\mathrm{A} \beta$ is at present unknown. In this study, we have examined the effect of APP in mouse N2a cell line, which capable of differentiating into neuron-like cells. N2a cell was stably transfected with wild-type (wt) and FAD mutant forms of APP (APPswe). Levels of secreted and intracellular A $\beta_{1-40}$ and $\mathrm{A} \beta_{1-42}$ were measured by ELISA. Compared to cell line expressing APPwt, both secreted and intracellular $\mathrm{A} \beta_{1-40}$ and $\mathrm{A} \beta_{1-42}$ were sgnificantly increased in cell line expressing APPswe. Furthermore, We observed that both N2a cell line and APPwt expressing cell lines extended neuritis, while APPswe expressing cell lines did not extend neuritis. To detect if APP and $\mathrm{A} \beta$ affect N2a cell differentiation, serum was withdrawn for 12 hour to induce N2a cell differentiation. We found both wild-type N2a cell line and APPwt expressing cell lines extended much longer neuritis after differentiation, while APPswe expressing cell lines only extend very short neuritis. We also showed that after differentiation both N2a cell line and APPwt expressing cell lines were accompanied by an increase in the amount of phosphorylated microtubule-associated protein MAP1B, which has been associated with neurite outgrowth while no such change was observed in APPswe expressing cell line. Then we further detected whether intracellular or secreted $\mathrm{A} \beta$ affect differentiation. $100 \mathrm{nM}$ (about tenfold of the concentration of secreted $\mathrm{A} \beta$ in cell line expressing sweAPP) $\mathrm{A} \beta_{1-40}$ and $\mathrm{A} \beta_{1-42}$ were added to medium when $\mathrm{N} 2 \mathrm{a}$ cells induced to differentiation. We found $\mathrm{A} \beta_{1-40}$ and $\mathrm{A} \beta_{1-42}$ of this concentration did not affect cell differentiation. The results suggest that APP may have a role in cell differentiation. The reduction in differentiation in mutant cell lines may be due to the toxic effects of increased intracellular $\mathrm{A} \beta$.

47

Effect of Acute Hypoxia on Tau Protein Phosphorylation

R. Liu, X.C. Wang, X.G. Liu, et al. Department of Pathophysiology, Tongji Medical College, Huazhong University of Science and Technology, Wuhan 430030 P.R. China

This study is to explore the effect of acute hypoxia on tau phosphorylation. Rat brain was chopped into small nubs and incubated at $37^{\circ} \mathrm{C}$ for different time with or without oxygen supply. Then tau phosphorylation and expression was detected by Western blot. The results showed that significant phosphorylation of tau at Ser-396/Ser-404 was seen in normal Wister rat brain. The level of tau phosphorylation at the same epitopes declined by anoxic treatment. The results suggest that certain level of phosphorylation of tau at Ser-396/Ser-404 may be necessary for physiologic function of neuronal cell, and acute hypoxia induces dephosphorylation of tau.

Presenilin-1 may mediate Taupathy in Sporadic Alzheimer's disease

C.Q. Zhu. National Key Laboratory of Medical Neurobiology, Fudan University, Shanghai 200032, P.R. China

In Alzheimer's disease brain, the most prominent pathological changes are included taupathy related neurofibrillary tangles (NFTs), amyloid-rich senile plaques and the massive selected neuronal loss. Mutations on the presenilin (PS) genes, has been known, causes an early onset form of Family Alzheimer's disease. For studying whether PS1 is associated with neuropathological changes of Sporadic Alzheimer's Disease (SAD), immunohistochemistry, immunoblot, co-immunprecipitation and PS-1 cDNA vector transfection 
methods were employed in present study. Results showed that PS-1 was expressed higher level in neurons of neocortex, hippocampus and cerebellum, and the PS1 immunopositive (PS1-IR) punctate matter appeared predominan somatodendritc localization in neurons of normal human brain or rat brain. In SAD brain, PS1-IR signal not only existed in several types of senile plaques, but also distributed in degenerating neurons without punctate form. Moreover, under confocal microscopy, PS1-IR co-localized with a subset AT8 (an anti-phosphorylated tau antibody) positive intraneuronal NFTs was observed in SAD brain. To confirm the relationship between PS1 and tau protein, we applied tau protein expressed neuroblastoma cell line NG108 to do PS1 cDNA transfecting study. After PS1 cDNA transfecting, the phosphorylated tau protein increased in NG108 cells which detected by immunblot and immunostaining, and the subcellular co-localization of PS1-EGFP and phosphorylated tau protein appeared in some transfected cell. The co-immunprecipitation study manifested that PS1 can directly bind hypophosphorylated tau protein extracted from SAD brain. These results implicated roles of PS1 in regulating tau protein phosphorylation and in phosphorylated tau aggregation to form NFTs in SAD.

49

Effect of overproduction of $\beta$-amyloid protein on neuronal cytoskeleton Z.F. Wang, X.C. Wang, J.Z. Wang. Department of Pathophysiology, Tongji Medical College, Huazhong University of Science and Technology, Wuhan 430030, P.R. China

Alzheimer's disease pathology is characterized by extracellular amyloid plaques and intracellular neurofibrillary tangles. $\beta$-amyloid protein $(\mathrm{A} \beta)$, a $39-43$ residue peptide deriving from the proteolytic processing of the amyloid precursor protein (APP), is the main proteinaceous component of amyloid plaques and plays a center role in the pathogenesis of $\mathrm{AD}$. However, the physiological function of $\mathrm{A} \beta$ and the relationship between $\mathrm{A} \beta$ and phosphorylation of cytoskeletal proteins are poorly understood. Since the Swedish mutant APP enhances $\beta$-secretase cleavage with a concomitant excess $\mathrm{A} \beta$ production, the level of $\mathrm{A} \beta$ is different among the three cell lines, N2aWT (wild type N2a cell), N2a695wt (stably transfected with human wild type APP695), N2aSwe (stably transfected with human Swedish mutant APP695). In this study, we observed significant morphological difference in three cell lines, and the difference was enhanced after treatment with butyric acid (BA). Immunocytochemistry study showed that phosphorylated NFs (detected by SMI31 and SMI34) were mainly located at cell progresses and non-phosphorylated NFs (detected by SMI32 and SMI33) predominantly distributed in cytoplasm. More interestingly, only the staining of SMI33 was positive with N2a695wt. Further data obtained from Western blot indicated that both phosphorylated and non-phosphorylated $\mathrm{NFs}$ were reduced in N2aSwe cell lysate in a time-dependant manner after BA treatment, whereas there were no detectable NFs in N2a695wt cell lysate with any of the four antibodies. It is suggested $\mathrm{A} \beta$ may play a role in neuronal differentiation and may reduce the expression of NF.

50

Intracerebral Transplantation of TH-transfection/Engineered Cell in Treating Monkey with Parkinson' disease

C.Y. Wu, J.G. Wang, Y. Yang, et al. Department of Neurosurgery, Qilu Hospital, Shandong M University, Shangdong 250012, P.R. China

Our aim is to transfect recombined plasmid with human TH gene to neurobastocytoma cell to construct cell lines expressing TH gene. After being capsulated, they were transplanted into brains of monkey models. By efficient and stable expression, TH deficiency of Substansia Negro was corrected, and DA concentration of striatum increased to a therapeutic level. Cloned E. Coli with pcDNA3/hTH plasmid were transfected into human neuroblastomacyte line SY5Y. Positive clones selected by G481 were cultured. TH-decorated cells dealt by microcapsulating were then transplanted into PD monkey models. Behavior and DA concentration in CSF were observed. Transplanted cells were also examined by immunocytochemistry to determine their living status. Subcloned and purified plasmids with $\mathrm{pcDNA} 3 / \mathrm{hTH}$ gene were resolved into fragments of $1.9 \mathrm{~Kb}$ and $5.5 \mathrm{~Kb}$ by ECORI enzyme, which was in accordance with anticipated result. So recombined plasmids were testified to be right. Immunohistochemical staining of transgenetic SY5Y cells showed strong positive. Symptoms of post-transplanted monkeys improved dramatically. DA concentration on CSF also increased. Large numbers of transplanted TH-stained positive gliocytes were found in transplanting areas using SABC immunocytochemistry. Therefore, human neuroblastoma SY5Y cells constructed through gene transfection can express human TH gene in vitro and posttransplantation. Microcapsulated trangenetic cells can live well in monkey's brain and perform therapeutic effect.
Y.C. Wu, R.S. Xu, E. Tong, et al. Department of Neurology, the First Affiliated Hospital of Jiangxi Medical College, P.R. China

The purpose of this study is to study the secondary damage mechanism of the residual dopaminergic neuron in midbrain nigra, the bcl-2, bax (bcl associated $\mathrm{X}$ protein), nNOS (neuronal nitric oxide synthase) protein expression of the residual dopaminergic neurons in midbrain nigra was observed in the normal control and PD group treated by immunohistochemical means. By computerized image analysis, the light absorption degress (A) of bcl-2, bax-2, bax, nNOS positive cells were measured and analyzed. The bcl-2 protein expression of the residual dopaminergic neurons in right midbrain nigra is significantly lower, and the bax, nNOS protein expression significantly higher than that of the left nigra dopaminergic neurons in PD group and both side of normal control group $(P<0.01)$, There is not significant difference among the bcl-2, bax and nNOS protein expression in left or right lateral nigra dopaminergic neurons in the normal control group midbrain $(P<0.05)$. The excessive apoptosis induced by pathologic factor (e.g. NO) in residual dopaminergic neurons in midbrain nigra is one of their secondary damage mechanism.

Effects of pinealectomy on the apoptosis of hippocampus and related gene expression induced by amyloid $\beta$ protein

B. Xu, J.P. Chen, Z. Fu, et al. Department of Neurology, 187 Hospital of PLA, Haikou 571159, P.R. China

The purpose of this study is to investigate effects of decreased melatonin (MT) on the apoptosis and the apoptotic-related genes expression induced by amyloid $\beta$ protein $(\mathrm{A} \beta$ ) following the pinealectomy. Rats were divided into two groups randomly. The experimental group was performed by pinealectomy, the control was made sham operation. Amyloid $\beta$ protein 1-40 $\left(\mathrm{A} \beta_{1-40}\right)[10 \mu \mathrm{g}(10 \mu \mathrm{g} / \mu \mathrm{l})]$ was injected into the hippocampus of the rats in the both groups above, at fortieth days after pinealectomy operation. The brain tissues were stained by progressive Corge Red staining and the apoptotic cells were examined by HE staining and terminal deoxynucleotidyl-transferase mediated d-UTP-biotin nick end labeling (TUNEL) after injection seven days, while $\mathrm{Bax}, \mathrm{Bcl}-2$ protein expression of apoptotic-related gene were also studied by SABC method. We observed: the apoptotic cells with HE staining $(16.37 \pm 5.3$, cells/field $)$ and TUNEL method (38.92 \pm 8.81 , cells/field) in the experimental rats were much more than the ones with corresponding staining $(7.29 \pm 2.5,20.63 \pm 5.28)$ respectively $(P<0.01)$ and the Bax protein expression $(14.28 \pm 4.18)$ were more strong than the $\operatorname{Bax}(7.69 \pm 2.81)$ in the control $(P<0.05)$, but there was not different between the experimental and the control group in Bcl-2 protein expression (7.48 $\pm 3.26,7.19 \pm 3.37)$ $(P>0.05)$. The results show pinealectomy of rats resulted in more apoptotic cells and strong its related gene expression of Bax protein induced by $\mathrm{A} \beta_{1-40}$.

53

Okadaic Acid Induces Neuronal Mitotic protein Expression in Adult Rat Brain

D.J. Hong, S.D. Lu, C.Q. Zhu. National Key Laboratory of Medical Neurobiology, Fudan University, Shanghai, 200032, P.R. China

Recent evidences implied that the development of Alzheimer disease (AD)type pathology was the consequence of an aberrant re-entry of post-mitotic neurons into the cell division cycle. It was well known that phosphatase activity decreased in AD brains, which at least partly contributed to tau overphosphorylation in AD. We wondered whether the inhibition of phosphatase could lead to cell cycle disturbance in differentiated brain neurons. In the present study, we examined cyclin B1 and p35 expression in post-mitotic neuron with immunohistochemistry after unilateral injection okadaic acid (OA, an inhibitor of PP1 and PP2A) into frontal cortex of adult rats. We observed the cyclin $\mathrm{B} 1$ and $\mathrm{p} 35$ positive neurons in the ipsilateral cortex as early as $4 \mathrm{~h}$ after OA injection, but less cyclin $\mathrm{B} 1$ positive glia cells and without $\mathrm{p} 35$ positive glia cells. The distribution of cyclin B1 positive cells also gradually expanded to other brain regions such as contralateral cortex, hippocampus and entorhinal cortex, besides the injecting site of frontal cortex. The number of cyclin B1 positive cells reached climax at 7 , and still left at $28 \mathrm{~d}$. Compared with the control group, the expression of p35 slightly increased at $4 \mathrm{~h}$, then reached the expression peak at $24 \mathrm{~h}$. In addition, we noticed that nucleus intensively stained with $\mathrm{p} 35$ antibody in some neurons, but less cyclin B1 positive nuclei. To study the relationship between mitotic proteins and tau protein phosphorylation, double immunostaining method was employed. Under confocal microscopy, we observed that cyclin B1 and p35 fractionally co-localized with overphosphorylated tau labeled by AT8 antibody in the cortical neurons. Our data indicated that mitotic protein expression could be induced in post-mitotic neurons and the relationship between the mitotic protein expression and the imbalance of neuronal kinase-phosphotase system may contribute to taupathy in $\mathrm{AD}$. 

Cytoprotective effect of melatonin on okadaic acid induced Alzheimer-like lesion

X.T. Li, Z.L. Wei, X.C. Wang, et al. Tongji Medical College, Huazhong University of Science and Technology, Wuhan 430030, P.R. China

To explore the protective agent(s) against Alzheimer-like abnormal phosphorylation of neuronal cytoskeletal proteins. An Alzheimer-like protein phosphatase deficient model was made by culturing neuroblastoma cell with okadaic acid. The effect of several recognized antioxidants, such as melatonin, Ginkgo biloba and estrogen on cell activity, phosphorylation and accumulation of neurofilament proteins was determined by MTT, crystal villain and immunocytochemistry techniques. Dose-dependent neurites degeneration and cell deaths were seen after okadaic acid treatment. Addition of melatonin to culture medium along with okadaic acid significantly prevented this cell toxicity. In addition, exposure of neuroblastoma cells to okadaic acid induced an increased staining in phosphorylated neurofilament $\mathrm{H} / \mathrm{M}$, and a concomitant decreased level of non-phosphorylated neurofilament subunits in the cell body and a condensed staining of the neurofilaments at the proximal end of cell processes. A disruption of microtubule system was also seen in okadaic acid treated cells. The hyperphosphorylation, accumulation of neurofilament subunits and disruption of microtubules caused by inhibition of protein phosphatase was remarkably prohibited by melatonin. No protective effect was detected by using different concentrations of Ginkgo biloba or estrogen. In conclusion, melatonin protects neuronal toxicity mediated by Alzheimer-like protein phosphatase deficiency. And the protective effect of melatonin is not substituted by anti-oxidant agents Ginkgo biloba or estrogen.

55

A Study on tau and $\beta$-tubulin expressions in the brains of Alzheimer's

J. Xu ${ }^{1}$, C.Y. Qian ${ }^{2}$, Y.Y. Fang ${ }^{2}$, et al. ${ }^{1}$ Department of Neurology, First Affiliated Hospital; ${ }^{2}$ Sun Yat-sen University of Medical Sciences, Guangzhou 510080, P.R. China

The objective is to reveal the correlations between the tau and $\beta$ tubulin antigen expressions, distributions and neuron skeleton deformity in the Alzheimer's disease (AD) patients' brains. Methods by immunohistochemistry (IHC) technology, the expression of the tau and $\beta$-tubulin antigen at different regions of hippocampus, cerebral cortex and olfactory ball in 9 cases of $\mathrm{AD}$ and 5 cases of non-dementia (ND) were observed and semi-quantitive analyzed. Results in AD brains, tau antigen expression greatly increased, which intensely stained the neurofibrillary tangles, neuropil threads, and neuritic plaques with more prevalence in hippocampus, temporal or frontal cortex. In ND cases, $\beta$-tubulinimmunoreactivity was easily visible in apical dendrites of hippocampal pyramidal neurons as well as some neocotical pyramidal neurons, some axonal fiber tracts were also stained in white matter. However, in AD, the number of immunoreactive dendrits in those areas reduced greatly. Only a few neurons can be stained by the two antibodies, and the correlations between tau and $\beta$-tubulin were irrelevant. Conclusions: In AD, tau immunopositive structures induced greatly with regional pattern differences. Moreover, the reduction of $\beta$-tubulin expressions indicated the normal cytoskeletal elements had destroyed or developed other abnormal strctures.

56

The Action of Raising Effects and Reducing Toxins by Nourishing the Liver and the Kidney, Dredging the Channels and Relieving the Toxins on Parkinson's disease

C.X. Yuan, Shanghai Longhua Hospital Attached to Shanghai University of TCM, 200032 P.R. China

Parkinson's Disease (PD) is the disease of degeneration of nervous system, commonly seen in the middle and old-age people. After 5 to 20 years' treatment of L-dopa preparation, side effects will appear certainly. According to the experience of famous doctor $\mathrm{HU}$ jianhua, on the base of the original therapy of anti-PD by the same dosage and the method of western medicine, the patients were treated by herbal medicine of nourishing the liver and the kidney, dredging the channel and relieving the toxins in addition. The open research on 30 cases of PD showed that the total effective rate was $53.33 \%$ and the improved Webster scores reduced from $16.77 \pm 3.98$ to $11.5 \pm 3.80(t=7.77$, $p<0.001) .10$ patients had on-off phenomena, and the average on time was delayed from $6.00 \pm 0.94$ hours to $7.85 \pm 0.06$ hours $(t=2.37$, $p<0.05$ ) when they were awake. It obviously improved the constipation, gastrointestinal symptoms, dizziness and sleeplessness. So it indicated that the traditional Chinese Medicine could increase.

57

Primary Signal of Vascular Dementia in Senitity- Diffuse White Matter
Lucencice

X.C. Zao, X.C. Wen. Department of Neurology, Tianjin Huanhu Hospital, Tianjin 300060, P.R. China

The aim of this study is to analyse the relationship between vascular dementia in senitity and diffuse white matter lucencice, and to observe 54 casesc of diffuse white matter lucencice for the patient over sixty for three years running with methods of clinic and CT treatments. The results show that 33 casesc of the above 54 casesc are vascular dementia in senitity, among which, 6 casesc in the first years, 13 casesc in the secondary years, 33 casesc in the third years. In conclusion, diffuse white matter lucencice is a critical factor of vascular dementia in senitity. The positive correlation between the degree of diffuse white matter lucencice and the occurrence of vascular dementia in senitity has shown. The active prevented and early treated for diffuse white matter lucencice should be recommended.

58

Changes of Thioredoxin mRNA Level in Neurons Insulted by Sodium

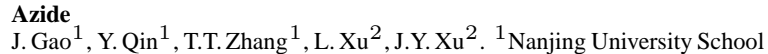
of Medicine, 210093; ${ }^{2}$ Nanjing Military Medical College, 210099 P.R. China

Sodium azide $\left(\mathrm{NaN}_{3}\right)$ is a specific inhibitor of mitochondrial cytochrome c oxidase (COX), which can be used to mimic neuronal damage induced by mitochondrial deficiency. Thioredoxin (Trx) is one of the potential endogenous antioxidants in the brain and is also a key protein for the control of cellular redox status. In this experiment, the neurotoxic effects of $\mathrm{NaN}_{3}$ on cultured primary neurons were detected by means of cell viability measurement (MTT assay) and morphological observation, and an in vitro model of neuronal injury induced by $\mathrm{NaN}_{3}$ were established. Further the changes of thioredoxin mRNA level in cultured neurons insulted by $\mathrm{NaN}_{3}$ were analyzed by semiquantitative RT-PCR in order to explore the role of Trx in the process of neuronal injury. It was found that cultured neurons could be damaged by $\mathrm{NaN}_{3}$ in a dose- and time-dependent manner and the expression of Trx decreased during certain dose $(0-32 \mathrm{mmol} / \mathrm{L})$ and time $(0-4 \mathrm{hr})$ of $\mathrm{NaN}_{3}$ treatment. The above data suggest that decreased the expression of Trx may be associated with oxidative stress induced by neuronal respiratory chain injury, which could impair neuronal protective mechanism.

59

Olfactory Identification is associated with Apolipoprotein $\mathrm{E} \varepsilon 4$ allele in mild cognitive impairment

Q.S. Wang ${ }^{1,2}$, S. Qin ${ }^{1}$, J.N. Zhou ${ }^{1,3}{ }^{1}$ Lab of Neurodegenerative Diseases, School of Life Science, University of Science and Technology of China, Hefei, 230027; ${ }^{2}$ Department of Neurology, the 105th Hospital of PLA, Hefei, 230021, ${ }^{3}$ Anhui Geriatric Institute, the First Affiliated Hospital of Anhui Medical University, Hefei 230027, P.R. China

The objective was to investigate olfactory identification and apolipoprotein $\mathrm{E} \varepsilon 4$ allele in patients with mild cognitive impairment. We used CC-SIT (from University of Pennsylvania) to assess olfactory identification and PCR to detect apolipoprotein $\mathrm{E} \varepsilon 4$ allele in 20 patients with mild cognitive impairment patients and the 30 aged-matched control subjects. We compared the performance on BST of the $20 \mathrm{MCI}$ patients and the 30 aged-matched control subjects. The Mann-Whitney $\mathrm{U}$ test demonstrated relative to control group $(P<0.0001)$. For MCI patients there was a significant relation between performance on CCSIT and CAMCOG-C $(r=0.65, p<0.05)$. As the least common allele in Chinese, $\varepsilon 4$ was found in $13.3 \%$ of controls and in $35 \%$ of MCI in this study. ApoE $\varepsilon 4$ was significantly increased in MCI group versus Normal group $\left(x^{2}=4.65, p<0.001\right)$. There is the significant effect of allele status on odor identification: group with $\varepsilon 4$ allele was not able to identify as many odors as the group without $\varepsilon 4$ allele ( $p<0.001$ ). In conclusion, there is a marked deficit in olfactory identification performance in patients with mild cognitive impairment. Olfactory Identification is associated with Apolipoprotein E $\varepsilon 4$ allele in mild cognitive impairment.

60

Therapeutic Evaluation of Glomus cells of Carotid Body Grafts for Hemiparkinsonian Rats: Behaviors and Neurotransmitters

X.B. Cao, S.G. Sun, T. Wang. Union Hospital, Tongji Medical college, Huazhong University of Science and Technology, Hubei, Wuhan 430022 P.R. China

The aim is to observe the effects of glomus cells within carotid body transplantation in a hemiparkinsonian rat model. Rats with unilateral 6hydroxydopamine lesions of the right dopaminergic neurons of substantia nigra received intrastriatum grafts of homo-or heter-intrastriatum glomus cells within carotid body and fetal mesencephalic neurons respectively. Apomorphineinduced rotation was monitored for 40 min each time 2, 4, 8 and 12 weeks after 
grafting respectively. The striatum tissues were cut for Glu, Gly, Asp, GABA and dopamine contents analyzed by HPLC at the end of the experiments. The results were that, compared with the control group, apomorphine-induced rotational behaviors were significantly reduced for 12 weeks and the dopamine contents were significantly elevated, especially in homo-graft group whose contents of Glu and GABA were decreased after grafting $(P<0.01)$, respectively. The present study demonstrates that intrastriatum grafts of glomus cells within carotid body in rats with 6-OHDA lesions can reduce apomorphineinduced rotational behavior and elevate the dopamine contents, decrease the Glu and GABA contents within striatum, is an effective method for PD.

61 $5-\mathrm{HT}_{2 \mathrm{C}}$ receptor and Safflor yellow modulate production of secretory amyloid $\beta$-protein precursor in incubated rat hippocampal slices

G.R. Zhang ${ }^{1}$, L.G. Cao $^{1}$, M.L. Guo ${ }^{2}$, et al. ${ }^{1}$ Department of Pharmacology, College of Pharmacy, Second Military Medical University, Shanghai 200433, P.R. China; ${ }^{2}$ Department of Pharmacognancy, College of Pharmacy, Second Military Medical University, Shanghai 200433, P.R. China

The study aimed To determine whether serotonin, a major neurotransmitter in brain, and Safflor yellow, could modulate the production of the secretory amyloid $\beta$-protein precursor (sAPP) by activation of serotonin $5-\mathrm{HT}_{2} \mathrm{C}$ receptor. The hippocampal slices of rats were incubated with various concentration of serotonin, $5-\mathrm{HT}_{2} \mathrm{C}$ agonist $\mathrm{M}-110$, or $5-\mathrm{HT}_{2} \mathrm{C}$ antagonist $\mathrm{L}$ 107 , or Safflor yellow. SAPP released into the incubation medium were assayed by western blot analysis assay with monoclonal antibody $22 \mathrm{C} 11$ for $2 \mathrm{~h}$. In various concentrations, serotonin (from $0.01 \sim 1000 \mu \mathrm{mol} / \mathrm{L}$ ) and M-110 $\left(1.5 \times 10^{6} \sim 1.5 \times 10^{3} \mu \mathrm{mol} / \mathrm{L}\right)$ had positive effects on the production of sAPP, while L-107 $\left(1.0 \times 10^{-9} \sim 1.0 \times 10^{3} \mu \mathrm{mol} / \mathrm{L}\right)$ had negative effects on the production of sAPP over controls. Safflor yellow $\left(1.0 \times 10^{-7} \sim 1.0 \times 10^{3} \mu \mathrm{mol} / \mathrm{L}\right)$ showed a biphasic actions (increase at small doses and decrease at large doses) in the modulation of the release of sAPP. In conclusion, serotonin modulates production of secretory amyloid $\beta$-protein precursor through serotonin $5-\mathrm{HT}_{2 \mathrm{C}}$ receptor in incubated rat hippocampal slices. Safflor yellow showed a biphasic actions in the modulation of the release of sAPP, which deserves further exploration.

62

Oxidative DNA damage in Alzheimer's disease brain

J.J. Zhang ${ }^{1}$, Y.Z. Wang ${ }^{2}$. ${ }^{1}$ Department of Neurology, Zhongnan Hospital, Wuhan University 430071, P.R. China; ${ }^{2}$ Department of Neurology, Anhui Provincial Hospital, Hefei 230000, P.R. China

There is increasing evidence indicate that oxidative stress is a contributor to neuronal death in Alzheimer's disease (AD). The oxidative damage that occurs to DNA may play a role in AD. This is a study of levels of 8-hydroxy2'-deoxyguanosine (8-OHdG), the marker of oxidative DNA damage, in CSF 8 -OHdG was isolated from CSF from $11 \mathrm{AD}$ and 5 age-matched control subjects and measured using gas chromatography/mass spectrometry. statistically significant elevations $(p<0.05)$ of 8-OHdG compared with age-matched control subjects. Our results support the concept that the brain is under increased oxidative stress in AD oxidative DNA damage could be involved in the pathogenesis of neurodegeneration in $\mathrm{AD}$ and 8-OHdG may be useful as a marker.

63

A Study of vascular dementia and white matter change of the brain Zhang Shuangguo, Wu Danhong, Liu Zhicao et al. Department of Neurology, Renmin Hospital of Wahan university, Wuhan 430060, China.

The purpose was to investigate the white matter change (WMC) in the patients with vascular dementia (VD), and explore the mechanism of the dementia caused by WMC. There were 84 patients with multiple infarct dementia, ie, VD group, including 48 males and 36 females, with mean age of $65.5 \pm 7.2$ years. Ninety-five cases of multiple infarct without dementia (54 males and 41 females with mean age of $61.1 \pm 8.3$ years) were served as controls. According to the scope of brain WMC, the degree of WMC was defined as "slight" (less than quarter of white matter), "moderate" (half to quarter) and "severe" (more than half, combining to flake). Hasegawa dementia scale (HDS) was used to evaluate the patients. Those with HPS score less than 10 were defined as severe dementia; $21.5 \sim 10.5$, moderete; $30.5 \sim 22$, slight; more than 31 , normal. The results showed the the VD patients showed remarkable WMC, there was significant difference compared with the controls $(p<0.01)$. The VD patients showed different extent of WMC and different degree of dementia, and the extent of WMC was positively related with the degree of dementia. Conclusion: The WMC in the dementia patients was an important cause in the dementia, MWC contributed to the occurrence and development of dementia in the VD patients. It was suggested that the dementia resulted from WMC. correlated with the disconnection among white matter, cortex and intercortex caused by the lesions of lateral periventricle.

\section{Cellular and Animal Models, Pharmacology, Neuroprotection}

\section{4}

Pharmacological enhancement of dentate gyrus neurogenesis and learning and memory

I. Grundke-Iqbal, Y. Tatebayashi, M.H. Lee, K. Iqbal. New York State Institue for Basic Research in Developmental Disabilities 1050 Forest Hill Road, Staten lsland, New York 10314-6399, USA.

Alzheimer disease (AD) is characterized clinically by cognitive deficit and behavioral abnormalities including mood disturbances. We investigated immunocytochemically the involvement of the dentate gyrus neurogenesis in AD. Musachi-1, a marker for undifferentiated progenitor cells, was found to be increased in the entire granular/subgranular layers of the dentate gyrus. Furthermore, FGF-2 and tau were codistributed and upregulated while the expression of MAP2 was decreased indicating a dendrite to axon polarity shift. Cerebrolysin (CL), a clinical drug, which has been shown previously to improve cognition and mood of AD patients, was found to increase neuron-like differentiated adult rat hippocampal progenitors in culture both by reducing spontaneous apoptosis and by counteracting the FGF- 2 induced polarity shift. CL increased MAP2, decreased glycogen synthase kinase- $3 \beta$-phosphorylated tau levels and enhanced synaptogenesis. Intraperitoneal administration of CL enhanced dentate gyrus neurogenesis and maze performance of 8- to 12-monthold female rats. These studies suggest that the pathogenesis of AD probably involves a dysregulation of denate gyrus neurogenesis. This pathology, which might be associated with abnormally elevated FGF-2 and with clinically reversible cognitive/mood symptoms, is a promising therapeutic target for $\mathrm{AD}$.

65

Analysis of Mouse Model Exhibiting Neurofibrillary Changes

A. Takashima. Laboratory for Alzheimer's disease, Brain Science Institute, RIKEN

Dysfunction and filament formation of microtubule-binding protein tau are key markers of neurodegeneration, including Alzheimer's disease (AD). Immunocytochemical studies show that neurofibrillary tangles (NFTs) are composed of highly phosphorylated tau. Congo-red birefringency, thioflavin-S reactivity, and argyrophilia in NFT-bearing neurons also demonstrate that the tau aggregation forms fibrils with a $\beta$-sheet structure. Discovery of the molecular mechanisms of NFT formation may lead to more insight about events occurring during neurodegeneration. In frontotemporal dementia parkinsonism 17 (FTDP17), genetic studies indicate that tau is a causative gene, and mutation is found in exons and introns of tau gene. The patient, who possesses this mutation, exhibits pathologically NFT and clinically personality change and cognitive dysfunction. Then, we produced the $\mathrm{Tg}$ mice expressing human longest tau with misssense mutation V337M and R406W. In the present study, neurons of hippocampus in our Tg mice showed phosphorylated tau accumulation with a $\beta$-sheet structure. This was demonstrated by Congo red, thioflavin-S positive staining, and argyrophilia a histological criterion used to identify NFTs observed in neurodegenerative disorders. The mice also displayed the reduced neural activity in hippocampus and altered behaviors that were associated with NFT formation. Thus, the results suggested that mutant tau expression induced intraneuronal tau accumulation leading to the reduction of neural activity and alteration of behavior in mice.

Cerebrolysin in Alzheimer's disease: A Randomized, Double-blind, Placebo-controlled Trial with a Neurotrophic Agent

E. Ruther ${ }^{1}$, S. Kasper ${ }^{2}$, H. Moessler ${ }^{3}$. 1University Clinic for Psychiatry, Goettingen, Germany; 2 Vienna General Hospital-University Clinic for Psychiatry, Vienna, Austria; 3EBEWE Arzneimittel GmbH, Unterach, Austria

Cerebrolysin is a peptide preparation with neurotrophic activity. It consists of low molecular weigth petides and free amino acids. In earlier clinical trials, it has been shown to be effective in the treatment of Alzheimer's disease (AD) after only one month of active therapy. In the present study, the effects of repeated treatments with Cerebrolysin were investigated in patients with mild to moderate $\mathrm{AD}$. In this multicenter, randomized, double-blind, placebocontrolled, parallel-group study, patients received IV infusions of $30 \mathrm{ml}$ Cerebrolysin or placebo five days per week for four weeks. This treatment regimen 
was repeated after a two-month therapy-free interval. Effects on cognition, global function, activities of daily living, and behaviour were evaluated with the Alzheimer's Disease Assessment Scale-Cognitive Subscale (ADAS-Cog), the Clinical Global Impressions (CGI), the Nuremberg Age Inventory, and the ADAS-noncog 4, 12, 16 (primary study endpoint), and 28 weeks after the beginning of the infusions. Of 147 patients enrolled, 71 were randomized to placebo, and 76 to CERE. A statistically significant group difference was observed at week 16 for the CGI $(P<0.004)$ and the ADAS-cog $(P<0.0001)$. In the CGI, $63.5 \%$ of Cerebrolysin but only $41.4 \%$ of Placebo patients responded to therapy (score $\leqslant 5$ ). In the ADAS-cog, Cerebrolysin patients improved by 2.1 points whereas Placebo patients worsened by 1.1 points from baseline, accounting for a treatment difference of 3.2 points in favour of Cerebrolysin. Significant improvement of Cerebrolysin patients was also evident in the activities of daily living and in behaviour. Notably, the clinical benefit observed at the end of the active treatment, was maintained to a large degree until the week 28 assessment, three month after druf withdrawal. Adverse events (AEs) were recorded in with a similar frequency in both treatment groups. Most common Aes were vertigo, headache, and sweating. In summary, Cerebrolysin treatment was well tolerated and leads to statistically significant and clinically relevant improvement of both cognition and global function. Sustained improvement of patients on Cerebrolysin was evident three month after drug withdrawal. These findings suggest that Cerebrolysin treatment may slow down the progression of the disease and that it exerts a stabilizing effect in $\mathrm{AD}$ patients.

67

Development of mtDNA-transfused Cell Models of Alzheimer's Disease and Parkinson's Disease and Application in Pharmacological Study on Chinese Herb Components

L. Li, F. Liu, L. Zhang, et al. Department of Pharmacology, Xuan-Wu Hospital of Capital University of Medical Sciences, Beijing 100053, P.R. China

The purpose of the present study is to develop mtDNA-transfused cell models (cybrids) of $\mathrm{AD}$ and $\mathrm{PD}$, to observe the pathological characteristics of the cybrid models, and to investigate the pharmacological effects of Chinese herb component SSY-P3 on the cybrid models. The platelets were isolated from 5 AD patients, 6 PD patients, 6 normal aged persons, and 6 normal young persons. The 0 cells without mitochondria were fused with the platelets under the induction of PEG 1500. The contaminated cells and unfused 0 cells were removed by replacing the medium with selective (uridine-free) medium. Cybrid clones were visible 10-15 days after fusion. Cytochrome c oxidase (COX) activity was determined by microplate assay. The specific fragment of mtDNA was detected by PCR and gel electrophoresis. The results showed: (1) The successful development of cybrids was verified by the evidence that cybrids grew and proliferated well in selective medium without uridine, recovered COX activity and had the mtDNA-specific fragment; but 0 cells failed. (2) The activity of COX in the AD cybrids decreased $29.1 \%$ and $45.7 \%(p<0.05)$ compared with aged control and young control group respectively; the COX activity in PD cybrids was $12.7 \%$ and $33.0 \%$ lower than that in aged control and young control respectively; the enzyme activity in aged control decreased $23 \%$ in comparison of young control group; (3) The level of ROS in AD cybrids was $44.9 \%$ and $63.5 \%$ higher than that in aged control and young control group respectively ( $p<0.05$ ); ROS in PD cybrids was $71.9 \%$ and $93.9 \%$ higher than that in aged control and young control respectively $(p<0.05)$ Incubation of Chinese herb component SSY-P3 $(100 \mu \mathrm{g} / \mathrm{ml})$ with AD and PD cybrids for $24 \mathrm{~h}$ declined their ROS production by $24 \%$ and $56 \%$ respectively. (4) MMP of AD and PD cybrids was respectively $46.5 \%$ and $47.8 \%$ lower than that of aged control $(p<0.01)$, and $48.7 \%$ and $49.9 \%$ lower than that of young control $(p<0.01)$. Incubation of $100 \mu \mathrm{g} / \mathrm{ml} \mathrm{SSY-P3} \mathrm{with} \mathrm{AD}$ and PD cybrids for $24 \mathrm{~h}$ elevated their MMP by $80.4 \%$ and $56 \%$ respectively $(p<0.05)$. (5) In comparison of young control, the basal cytosolic calcium of $\mathrm{AD}$ and $\mathrm{PD}$ cybrids was increased, and the regulatory ability to calcium was decreased. $24 \mathrm{~h}$ incubation of SSY-P3 with AD and PD cybrids decreased their basal cytosolic calcium and enhanced their regulatory ability to calcium. The results indicated that mtDNA-transfused cells (cybrids) of AD and PD can be used as a good model to investigate the pathogenesis related to mitochondria and to screen the drugs. Chinese herb component SSY-P3 may be beneficial to retarding or treating some neurodegenerative diseases.

68

Protective Effects for Adenoviral-mediated GDNF Gene Intracerebral Transfer in a Rat Model of Parkinson's disease

X.W. Chen, S.D. Chen, G.Y. Yang, et al. Department of Neurology, Clinic \& Research Center for PD, Ruijin Hospital, Shanghai Second Medical University, Shanghai 200025, P.R. China

In this study we explored the neuroprotective effects of adenoviral mediated GDNF gene transfer in the treatment of PD. 35 SD rats which were divided into
3 groups received perinigral injections of recombinant adenovirus encoding GDNF (Ad-GDNF) or LacZ (Ad-LacZ) or PBS respectively. One week later, intrastriatal injection of 6-OHDA was made to induce progressive degeneration of dopaminergic neurons. Our results showed Ad-GDNF-treated rats showed improved motor functions. The survival of $70 \% \mathrm{TH}$-positive cells in the lesioned substantia nigra was found in Ad-GDNF-treated rats, but the survival of $30 \%$ TH-positive cells in the lesioned substantia nigra was noted in AdLacZ- or PBS-treated rats. The content of DA in the lesioned striatum was much higher in Ad-GDNF-treated rats than that in Ad-LacZ- or PBS-treated rats. RT-PCR and ELISA analyses demonstrated the efficient expression of the exogenous GDNF gene in the midbrain. GDNF protein level in the ventral midbrain surrounding the Ad-GDNF injection site reached $1 \mathrm{ng} / 10 \mathrm{mg}$ wet tissue 5 weeks after the adenoviral vector delivery, which was 16-20 times of that of the Ad-LacZ- or PBS-treated groups. Our results suggest that Adeno-viral mediated GDNF gene intracerebral transfer significantly protect the dopaminergic neurons of nigrostriatal system from the 6-OHDA-induced injuries, and this therapeutic method be useful in the treatment of PD

69

Comparation of Three Neurodegenerative Animal Models Induced by Mitochondrial Deficit

L. Zhang, L. Li, R. Zhang, et al. Dept. of Pharmacology, Xuan-Wu Hospital of Capital University of Medical Sciences, Beijing 100053, P.R. China

Mitochondrial deficiency occurs in Pakinson's disease (PD), Huntington's disease (HD) and Alzheimer's disease (AD), we compared the difference of behavior, pathology and neuro-transmitters among these 3 neurodegenerative animal models to study the mechanisms of the diseases. C57BL mice were treated with MPTP (mitochondrial complex I inhibitor, ip) to mimic PD; SD rats were treated with 3-NPA (mitochondrial complex II inhibitor, ip) to mimic HD; SD rats were administrated with $\mathrm{NaN}_{3}$ (mitochondrial complex IV inhibitor) subcutaneously via a Alzet minipump to mimic AD. Learning-memory ability,the movement ability. The number and morphology of neurons, the number and activity of dopaminergic neurons, the content of NE, dopamine, 5-HT and their metabolic products in striatum were investigated. MPTP mice showed damage of neurons in both substantia nigra and striatum, the content of dopamine and its metabolic product DOPAC also decrease in striatum. As the result, model mice indicated movement disorder without learning-memory deficit. 3-NPA rats showed damage of neurons in substantia nigra, striatum, cortex and hippocampus, but the content of neuro-transmitters didn't show any abnormality. Model rats indicated both movement disorder and learning-memory deficit. NaN3 rats showed damage of neurons in cortex and hippocampus, both neurons and neuro-transmitters in striatum didn't show any abnormality. So model rats indicated learning-memory deficit without any movement disorder. Different inhibition of mitochondrial complex induces the abnormality of neurons, neuro-transmitters and behaviors in different area of brain and results the different disorders. Although the mechanism of these results need the further study, these 3 animal models could be applicated in PD, HD and AD research because they mimic the abnormality in these neurodegenerative diseases.

70

A Experimental Study on Microelectrode-guided Pallidotomy for Treatment of Parkinson's disease

J.M. Hu, F. Shu, D.L. Xue, et al. Neurosurgical Department, Wuhan General Hospital, Guangzhou Command, PLA, Wuhan 430070, P.R. China

For the purpose of evaluating the validity of application of microelectrodeguided pallidotomy in the management of $\mathrm{PD}, 28$ successfully induced PD models in 40 rats by 6-OHDA injected into condense portion of substantial nigra (SNC) were divided randomly into 3 groups, the direct lesioning group,the microelectrode-guided pallidotomy group and the control group. The results showed that: significant therapeutic effects were present in both of the direct lesioning group and the microelectrode-guided Pallidotomy group $(P=0.034)$, and significant difference could be observed between them $(p=0.014)$. High successful rate can be obtained in establishing the PD models in rats by 6-OHDA injected into the SNC and this model may by the most cost-effective PD model at the present time. By the use of microelectrode, electriophysiological changes can be screened and the locating technique can be obviously improved during surgery for its better lesioning effect.

Improvement Effects of Huperzine-A on Cognitive Function and Disturbance of Conduct of Patients with Alzheimer's disease

Y.B. Jiang, S.S. Huang, L. Huang. Department of Neurology, Affiliated Psychiatric Hospital of Guangzhou Medical College, Guangzhou 510370, P.R. China

The purpose is to observe the effect of huperzine-A on cognitive function and disturbance of conduct of patients with AD. 33 patients with $\mathrm{AD}$ were 
treated with huperzine-A, the improvement degrees were observed in cognitive function and disturbance of conduct at 12th week, 24th week and 48th week, respectively. At the same time, side effects of huperzine-A and situation of using psychotropic drugs were remarked. After 12 weeks, 24 weeks and 48 weeks of the treatment, the improvement rate of cognitive function were $50.2 \%, 63.6 \%$ and $66.7 \%$ respectively, and there was no significant difference among three ones $(p>0.05)$. There was improvement of various degrees in disturbance of conduct also, and there was significant decrease in dosage of using psychotropic drugs comparing that at 24 th week with that at 4 th week $(p<0.05)$. Only five cases had side effects. The results demonstrated that huperzine-A was effective in stabilizing condition, improving cognitive function and disturbance of conduct of cases with $\mathrm{AD}$.

72

\section{The Effect on Function of DAT by Polyphenol}

T.H. Pan, Z.P. Liu, J. Zhang, X.D. Zhou, et al. Neurology Department, Renji Hospital, Shanghai Second Medical University, Shanghai 200001, P.R. China

The purpose is to know whether the Polyphenol had the effection on the uptaking of DA by Dopamine Transporter (DAT). The quantity of uptaking of DA by DAT on the cells transfected by DAT gene (CHO/DAT cells) and mice striatal synaptosomes was detected by the way of liquid scintillation counting. The effection on them by the Polyphenol was also detected. The results showed that CHO/DAT cells had the function of uptaking of 3H-DA. The quantity of uptaking of 3H-DA was much lower in Polyphenol-added group than that in non-Polyphenol group. It also showed that mice striatal synaptosomes had the function of uptaking of 3H-DA, and the quantity of which was also much lower in Polyphenol-added group than that in non-Polyphenol-added group both in Vitro and in Vivo. All the experiments above showed that Polyphenol might diminish the quantity of uptaking of 3H-DA and the quantity may diminish more significantly along with the increasing concerntration of Polyphenol. The result showed that Polyphenol might have the function of blocking DAT to uptake of 3H-DA. The results of this study suggested that Poyphenol may have some effects on the therapy of PD as an inhibitor of DAT and can offer a new field for the therapy of PD.

73

Comparison of Strategic Approach for the General Treatment of PD-Ldopa Monotherapy and Concomitant L-dopa Therapy with Dopamine Agonists

B. Qin, X.Y. Zeng, Y.P. Jiang, et al. Department of Neurology, Clinic \& Research Center for Parkinson Disease, Ruijin Hospital, Shanghai Second Medical University, Shanghai 200025, P.R. China

The clinical efficacy and safety of L-dopa monotherapy and concomitant L-dopa therapy with dopamine agonists (Bromocriptine or Pergolide) of the treatment of PD were studied. The clinical trial was performed in the multicentre, open-label study with a match of age and sex, randomized into 3 groups to receive treatment for 1 year. L-dopa group: 47 cases, L-dopa plus Bromocriptine group; 43 cases and L-dopa plus Pergolide group; 48 cases, all of them took L-dopa alone before enrollment. As adjunctive therapy to levedopa, Bromocriptine or Pergolide was administered with increased dose titration initially and maintained in satisfactory treatment dosage. The average daily dose of L-dopa in levedopa group was $523.3 \pm 235.9 \mathrm{mg}$, The average daily dose of L-dopa and Bromocriptine were $526.7 \pm 241.3 \mathrm{mg}$ and $7.3 \pm 1.5 \mathrm{mg}$ in L-dopa plus Bromocriptine group, respectively. The average daily dose of L-dopa and Pergolide were $558.3 \pm 192.9 \mathrm{mg}$ and $0.235 \pm 0.045 \mathrm{mg}$ in L-dopa plus Pergolide group separately. The clinical improvement was about $74.5 \%$ both in assessment of modified Webster's scale and MDRSPD in L-dopa group. The clinical score were improved in $69.8 \%$ (Webster's scale) and 79.1\% (MDRSPD) in L-dopa plus Bromocriptine group, respectively. The clinical improved rates were $77.9 \%$ (Webster's scale) and $81.3 \%$ (MDRSPD) in L-dopa plus Pergolide group. The incidence rates of side effects were $27.7 \%$ in L-dopa group, $39.5 \%$ in L-dopa plus Bromocriptine and $18.8 \%$ in L-dopa plus Pergolide groups. There was an efficacy in treatment of Parkinson's disease in either L-dopa monotherapy or combination with Bromocriptine or Pergolide. The treatment of L-dopa alone was more effective in the early stage of PD and concomitant L-dopa therapy with dopamine agonists were more effective in the advanced stage of PD, pergolide was not only more effective, safe and tolerable, but also fewer adverse events than Bromocriptine was in this short-term trial.

74

Injection of bradykinin A to hippocampus induces Alzheimer-like phosphorylation of Tau and abnormal behavior in rat

Q.B. Wang, J.Z. Wang. Pathophysiology Department, Tongji Medical University, Wuhan 430030, P.R. China

Bradykinin (BK) is a calcium/calmodulin dependent protein kinase II
(CaMK-II) specific activator. In vitro studies have shown that CaMK-II plays an important role in Alzheimer-like phosphorylation of microtubule-associated protein Tau. To reconstitute an animal model based on the imbalance of protein kinase(s) and protein phosphatase(s) seen in Alzheimer brain, we have injected BK into hippocampus, observed their behaviors by electronic attack-jump experiment and phosphorylation of Tau by immunohistochemical assay. The results from behavior study showed that an obvious disturbance in learning and memory was seen with BK injected rats. The results obtained by immunohistochemical assay indicated that the staining for M4, 12E8, PHF-1 and CaMK-II was stronger, and for Tau-1 was weaker in BK injected rats compared with control group. To our knowledge, this is the first data shown in vivo that the activation of CaMK-II induces both Alzheimer-like Tau phosphorylation and behavior disturbance.

75

Bilateral Injection of Isopreterenol into Hippocampus Induces Alzheimerlike Hyperphosphorylation of Tau and Abnormal Behaviours in Rat S. Li, X.C. Wang, S.Y. Liu, Q. Wang, I. Grundke-Iqbal, K. Iqbal, J.Z. Wang. Department of Pathophysiology, Institute of Neuroscience, Tongji Medical College, Huazhong University of Science and Technology

Abnormally hyperphosphorylated tau, which is the major proteinaceous constituent of the neurofibrillary pathology seen in Alzheimer's disease (AD), might be the result of an imbalanced regulation in protein kinases and protein phosphatases in affected neurones. Among the various protein kinases, cAMP-dependent protein kinase (PKA) might be a crucial candidate responsible for this hyperphosphorylation. To gain further insight into its abnormal phosphorylation on tau protein in vivo, we have injected isopreterenol (IP), a specific activator of PKA, into rat hippocampus bilaterally, thus leads to an Alzheimer-like imbalance in phosphorylation system. It was found by step down inhibitory avoidance task and water maze tests that IP-injected rats not only developed a one-trial step down inhibitory avoidance response deficiency but also an obvious disturbance in spatial memory retention. The data obtained by immunohistochemistry and Western blot indicated that tau was hyperphosphorylated at several Alzheimer-like epitopes. It is implicated that PKA participates in the regulation of tau phosphorylation in vivo and thereby may cause the impairment in memory. To our knowledge, this is the first data to associate the activation of PKA with Alzheimer-like tau hyperphosphorylation and behavioural abnormalities in vivo.

76

Animal Model of Alzheimer Disease Was Established and Acting Mechanism Researches of Zhinao Capsule on It

W.M. Yang, M.X. Han, Z.G. Li, et al. 1st Affiliated Hospital of Anhui College of TCM, Hefei 230031, P.R. China

In this study we used $\beta$-AP-injection into lateral ventricle and Transforming Growth Factor $\beta_{1}$-injection in the rat brain tissue to successfully make an animal model of $\mathrm{AD}$ with impairment of learning and memory, features of typical pathology and neurobiochemistry etc. Effects of Zhinao capsule on the indexes of behavious, neuropathology, neurobiochemistry and APP mRNA overexpression in rat brain had been investigated. The results showed that Zhinao capsule could: (1) improve the ability of learning and memory of AD model rats induced by $\beta$-AP, diminish the time of mean latency of rats, raise the percentage of light compartment retention and decrease the number and section area of $\beta$-AP deposition plaques in cerebral cortex and hippocampus CA1. (2) raise the activity of superoxide dismutase (SOD) and reduce the content of malondialdehyde (MDA) in the brain tissue of AD model rats. (3) enhance the activity of $\mathrm{ChAT}$ and the content of Ach and reduce the activity of AchE in cerebral cortex and hippocampus. (4) downregulate the level of APP mRNA overexpression in the brain tissue induced by interleukin- $1 \beta$. The results indicate that the effect of Zhinao capsule on $\beta$-AP way including three correlated link, APP mRNA expression, $\beta$-AP deposition and neurotoxicity caused by $\beta$-AP may be the main action pattern.

77

Risperidone on Behavioural and Psychological Symptoms of Dementia L.P. Xi, Q.M. Ji, Y. Liu, et al. The Second Mental Hospital of Wuhan, Wuhan 430084, P.R. China

The purpose is to study the efficacy of risperidone on behavioural and psychological of dementia patients. Before risperidone treatment and after 4 weeks, 8 weeks and 12 weeks, the efficacy of risperidone was evaluated by Mini-Mental State Examination (MMSE), Behavioura01 Pathology in Alzheimer's Disease Rating Scale (BEHAVE-AD) and Activity of Daily Living Scale (ADL). The side effects were assessed by Treatment Emergent Symptom Scale (TESS). After risperidone treatment, the total of MMSE increased significantly, and that of BEHAVE-AD and ADL decreased significantly. The side effect was slight. It suggests that riperidone is an effective and safe drug 
on behavioural and psychological symptoms of dementia.

78

The Pharmacological Researches of New Compound B20 for Senile dementia

D.H. Xu, L. Zhu, X. Feng, et al. Department of Pharmacy, Zhongshan University, Guangzhou 510275, P.R. China

In this research a new compound B20 having lower toxicity and stronger pharmacological functions, modified from starfish nature produce, was used to complete series of pharmacological researches to test its functions on brain memory, immune system and anti-oxidation. The experiment results show that B20 can markedly enhance the acquirement, strengthening and reappearance of memory, markedly improve the immune functions and the functions of anti-oxidation

The Protection of Polygonum Multiflorum Thunb to the Rats' Cerebral AChE Neurons Damaged by Kainic acid

M. Li, Q.D. Yang, X.P. Du. Department of Neurology, Xiang Ya Hospital, Xiang Ya Medical College, Central South University, Changsha 410008, P.R. China

This research is to observe the number and morphology changes of rats' basal forebrain cholinergic neurons under the destruction of Kainic acid and to investigate the protection and the mechnism of PMT to the Ach neurons and their fibers. To inject excitative neurotoxic Kainic acid to the BF Meynert neucleus, MS neucleus and the Broca neucleus (DB) in order to estabolish the destruction model, then feeding the destructive experimental group, use the histochemistry method to display the changes of AChE neurons and their fibers, also the protection and activation of PMT. The number of Meynert neurons in the BF in PMT feeding group A is larger than that of group B, and group A shows no cellular destruction. The number of projecting AChE fibers to the cortex and the hippocampus of group $\mathrm{A}$ is larger than that of group B, and group A shows no morphological destruction $(p<0.01)$. PMT has protective effect to the rats' $\mathrm{AChE}$ neurons and their projecting fibers.

80

Effect of Electroacupuncture on Cytoplasmic Free Calcium of Neurons and Content of Glu in Cornu Ammonis in Rat With Caudal-Putamen Hemorrahage

Ts. Tian, H. Wang, J. Ma. Hubei College of Traditional Chinese Medicine, Wuhan 430061, P.R. China

The research is to study the cytoplasmic free calcium of neurons and content of Glu in Cornu Ammonis in Wistar rats with caudal-putamen hemorrahage and the effect on above items of electroacupuncture. Collagense VII were used to induce caudal-putamen hemorrhage in rats. Neiguan, Shuigou, Housanli were electroacupunctured. The results showed: (1) Electroacupuncture could significantly improve the nerve missing sign of rats with caudal-putamen hemorrhage $(P<0.05)$. (2) Electroacupuncture could significantly reduce the cytoplasmic free calcium of neurons in Cornu Ammonis of the hemorrhage side $(P<0.01)$ which in model rats has been significantly higher than nomal level $(P<0.01)$. (3) Electroacupuncture could significantly reduce Glu contents in Cornu Ammonis on both sides $(\mathrm{P}<0.01)$ which in model rats have been significantly higher than nomal levels $(P<0.01$ or $P<0.05)$. Calcium overload and exicitotocity participate in the ischemic brain injury of Intracerebral Hemorrhage (ICH). Electroacupuncture has the effect against ischemic brain injury of intracerebral hemorrhage. The effect of electroacupuncture could be carried out by reducing the excitotoxic effect of Glu and high level of cytoplasmic free calcium of neurons in brain after ICH.

81

The Effect of Acupuncture on Brain Nucleus Protein and Non-histone Chromatin Protein(NHCP) in SAM-P/10

J.X. Han, P. Li, J.H. Zhao, et al. No. 1 Teaching Hospital of Tianjin College of TCM, Tianjin 3001931, P.R. China

In this research the protein pattern and scanning density of brain nucleus and chromatin protein bands on SDS-PAGE in SAM-P/10 were tested after acupuncturing Shuigou and Neiguan. The results showed the membrane protein with molecular of $62 \mathrm{KD}$ in nucleus protein which had rapidly increased in senescent progress of SAM-P/10 decreased obviously after acupuncture. Meanwhile the protein with molecular of $24 \mathrm{KD}$ and $66 \mathrm{KD}$ in chromatin protein which reduced with aging rised remarkably after acupuncture. The results suggest that puncturing Shuigou and Neiguan can regulate changes of some age-related proteins and improve functions of brain.

\section{Etiology, Epidemiology and Genetics}

82

Long-term follow-up of Alzheimer's disease and other dementias: Findings from a Population-based study

Hedda Agüero-Torres, Bengt Winblad, Laura Fratiglioni. Stockholm Gerontology Research Center, Division of Geriatric Epidemiology, Karolinska Institute, Sweden

The aim of this study is to increase understanding of the natural history of dementia in an old population. Data was derived from the Kungsholmen Project, a population-based study of subjects aged 75+ in Stockholm. Both prevalent and incident dementia cases were examined. Institutionalization, functional disability, cognitive decline and mortality were the main outcomes. The results showed: At baseline, 55\% of the demented subjects but only $3 \%$ of the non-demented were institutionalized. Demented people living in institutions were affected mostly by moderate-severe dementia and by vascular dementia (VD). The population attributable risk percentage of institutionalization due to dementia during the 3-year follow-up was $61 \%$ and both dementia and cognitive impairment were main contributors, independent of socioeconomic or functional status. In addition, dementia made the strongest contribution to the development of long-term functional dependence and decline in functioning, independent of the presence of other chronic diseases. Mini-Mental State Examination (MMSE) scores decreased by 2.4 points each year in demented subjects who survived 3 years and had an initial score $>5$. This decline was more rapid in those with higher initial MMSE scores, those with poorer functional status, and in AD. In this population, $70 \%$ of dementia cases died during the five years after diagnosis, with dementing disorders being a major risk factor. Even in the oldest old $(85+)$, dementia shortens life, especially among women. Fourteen percent of all deaths were attributable to dementia after controlling for other factors. Mortality risk ratios were 2.0 for $\mathrm{AD}(95 \%$ confidence intervals $=1.5-2.7)$ and 3.4 for VD $(95 \%$ confidence intervals $=$ 2.0-5.3). Shorter survival in dementia was related to male gender, advanced age, low education, comorbidity and initial poor functional status. Type, severity and duration of dementia were less important predictors. The results indicate that dementia is a long, progressive process, a major cause of functional dependence and institutionalization and a major risk factor for death in the elderly. Using clinical and demographic data to identify those with a worse prognosis is possible.

83

Incidence of Alzheimer's Disease and other Dementia Disorders. Findings from a Population-based Study of the Oldest Old in Stockholm, Sweden E. Von Strauss, H. Agüero Torres, B. Winblad, L. Fratiglioni. Stockholm Gerontology Research Center, Division of Geriatric Epidemiology, Karolinska Institute, Sweden

The aim of this study is to determine the dementia incidence in the very old in relation to age, gender and different dementia types. We used data gathered from a longitudinal population-based study carried out in Stockholm, Sweden. At baseline, the study population consisted of all persons aged $77+$ years registered in the Kungsholmen district, whether living at home or in institutions. During the follow-up, 131 subjects developed dementia. In the age group 77-84 years, the incidence rates for dementia were $43.9(95 \% \mathrm{CI}=$ $30.4-61.3)$ for women and $42.9(95 \% \mathrm{CI}=22.2-74.9)$ for men per 1,000 person-years, for 90-94 year old subjects the corresponding figures were 73.5 $(95 \% \mathrm{CI}=49.2-105.5)$ and $56.7(95 \% \mathrm{CI}=20.8-123.4)$ per 1,000 personyears. However, in the oldest age group $(95+)$ the incidence rates decreased for both women and men to $45.6(95 \% \mathrm{CI}=14.8-106.4)$ and $42.9(95 \%$ $\mathrm{CI}=1.1-239.1)$ per 1,000 person-years respectively. A similar pattern of distribution by age and gender was seen for Alzheimer's disease. In each age stratum, the incidence rates of dementia and Alzheimer's disease were higher for women than for men. The research indicates that the incidence of dementia increases with advancing age, but then peaks and declines for both genders in the very old (95+ years). This was especially evident among women, and is clearer for $\mathrm{AD}$

84

Differential evolution of non-demented older persons with cognitive impairment: Results from the Kungsholmen Project

K. Palmer, H.X. Wang, L. Bäckman, B. Winblad, L. Fratiglioni. Gerontology Research Center, Division of Geriatric Epidemiology, Karolinska Institute, Sweden

The research is to describe the evolution of Cognitive Impairment No Dementia (CIND).Results from the Kungsholmen Project showed: Of the 212 individuals with mild CIND, $63(34 \%)$ died, $65(35 \%)$ progressed to dementia, $21(11 \%)$ remained stable, and $46(25 \%)$ improved at first follow-up. The 
relative risks of progressing to dementia at first follow-up in subjects with mild, moderate and severe CIND were 3.6 (2.6-4.8), 5.4 (3.7-7.8), and 7.0 (4.5-10.8), respectively. The relative risks of death decreased with increasing severity of impairment. Individuals that improved at first follow-up did not have a significantly higher risk of later progressing to dementia than subjects who had never been impaired (RR: $1.4(0.7-3.0)$ ). The absence of a subjective memory complaint predicted improvement after adjusting for age, sex, and education (OR: 5.4 (2.0-14.9)). At the population level, CIND is a heterogeneous condition in which subjects may progress to dementia, death, or cognitive improvement in similar proportions in three years. There was no increased future risk of progressing to dementia in those CIND subjects who improved.

85

Late-Life Engagement in Social and Leisure Activities Decreases the Risk of Dementia: A Longitudinal Study from the Kungsholmen Project H.X. Wang, A. Karp, B. Winblad, L. Fratiglioni. Stockholm Gerontology Research Center, and Division of Geriatric Epidemiology, Neurotec, Karolinska Institute, Stockholm, Sweden

The aim of this research is to test the hypothesis that social support and intellectual stimulation may protect the elderly against dementia. A longitudinal population-based study using data from the Kungsholmen Project, which allowed us to examine whether current engagement in different activities $6.4 \pm 0.9$ years before dementia diagnosis was related to a decreased incidence of dementia. Three examinations have been completed in the project, with 3-year intervals (1987-1989,1991-1993, and 1994-1996). The subjects were 776 subjects who were $77+$ years old at the second examination of the project. At first examination, information on current engagement in social and leisure activities was obtained from all members of this cohort through a personal interview. At second examination, 776 subjects were still non-demented, and were followed for another 3 years to detect incident dementia cases (third examination). At each examination dementia cases were diagnosed by specialists according to the DSM-III-R criteria. The results showed: Frequent (dailyweekly) engagement in mental, social, or productive activities was inversely related to dementia incidence (adjusted relative risks, 95\% confidence interval for mental, social, and productive activities were $0.54,0.34-0.87 ; 0.58,0.37-$ 0.91 ; and $0.58,0.38-0.91$, respectively). The relative risks were adjusted for age, sex, educational attainment, cognitive functioning, depressive symptoms, as well as physical functioning at first examination. The three factors acted independent of each other. Stimulating activity, either mentally or socially oriented, may protect against dementia, both social support and intellectual stimulation are relevant for the mental health in the elderly.

86

The Association between ACE, MTHFR, Factor V and APOE Gene Polymorphism and Vascular Dementia

N.S. Wang, N.M. Bornstein, J. Chapman, T.A. Treves, A.D. Korczyn. Department of Physiology and Pharmacology, Department of Neurology, Tel Aviv Sourasky Medical Center, Sackler Faculty of Medicine, Tel Aviv University, 69978, Israel

In the current study we examined the usefulness of genetic risk factors for vascular disease as markers for vascular dementia (VD). The groups included 41 patients with VD, 49 patients with dementia of the Alzheimer's type, and 40 age-matched control subjects without dementia. These patients were genotyped for vascular disease-associated polymorphisms in the genes coding for methylenetetrahydrofolate reductase (MTHFR), angiotension-converting enzyme (ACE), factor V Leiden (FVL), and a common genetic risk factor for AD, ApoE 4. The results showed that there was no significant association between ACE, MTHFR and FVL geotypes with VD whether compared with subjects with $\mathrm{AD}$ or with control subjects. There was a high frequency of APOE e4 alleles in patients with $\mathrm{AD}(30 \%, p=0.016)$ and $\mathrm{VD}(26 \%, p=0.07)$ compared with control subjects $(15 \%)$. The results indicate VD is not associated with the genetic risk factors for vascular disease examined in this study, indicating that the pathogenesis of VD may differ from other vascular diseases.

87

Association between Low-density Lipoprotein Receptor-related Protein Gene, Butyrylcholinesterase Gene and Alzheimer's disease in Chinese

$\mathrm{S}$. Bi ${ }^{1}$, Y. Zhang ${ }^{2}, \mathrm{~J}_{\mathrm{Wu}}{ }^{2}$, et al. ${ }^{1}$ Department of Neurology, the First Clinical College of Harbin Medical University, Harbin, Heilongjiang; ${ }^{2}$ Department of Neurology, the First Clinical College of Norman Bethune University of Medical Sciences, Changchun, P.R. China

The purpose of this study is to study the relationships between lowdensity lipoprotein receptor-related protein gene (LPR) polymorphism, butyrylcholinesterase gene (BchE) polymorphism and $\mathrm{AD}$ in Chinese. The gene polymorphism of LRP and BchE was genotyped in $38 \mathrm{AD}$ cases and 40 con- trols with polymerase chain reaction-restriction fragment length polymorphism (PCR-RFLP) methods. AD groups were classified according to the LRP C/C genotype and compared with matched controls. The results showed that the AD group had higher frequencies of $\mathrm{C} / \mathrm{C}$ homozygote $(81.6 \%$ vs $60.0 \%$, $P<0.05$ and of $\mathrm{C}$ allele $(89.5 \%$ vs $76.3 \%, P<0.05$ ), but there was no significant difference between any of these $\mathrm{BchE}$ genotypes in classified AD groups and their respective control groups. These results indicate that there is a positive correlation between LRP gene polymorphism and $\mathrm{AD}$, but no relationships between $\mathrm{BchE}$ gene polymorphism and $\mathrm{AD}$ in Chinese $\mathrm{AD}$ cases.

The prevalence of senile dementia in the urban and the rural areas in Chengdu

M.N. Tang, X.H. Liu, X.Y. Zou, et al. Institute of Mental Health, The First Hospital of the West P.R. China University of Medical Sciences, Chengdu, 610041 P.R. China

The research is to study the prevalence of senile dementia and Alzheimer's disease in the elderly in the urban and the rural areas in Chengdu. This study was conducted among residents aged 55 years and over in the urban and the rural areas selected by stratified random sampling from four levels of 19 districts, cities and counties of Chengdu. The number of the elderly from the urban (or town) and the rural areas on each level was determined on the basis of the proportion of population of each level to population of Chengdu city. 5284 persons were examined with MMSE. 101 persons were examined by informant questionnaire because of deafness of blindness. The overall prevalence of senile dementia was $2.67 \%$. The prevalence of Alzheimer's disease (AD), vascular dementia (VD), mixtured dementia, Parkinson's dementia, dementia due to injury and alcoholic dementia was $2.06 \%, 0.37 \%, 0.06 \%, 0.11 \%, 0.02 \%$ and $0.06 \%$ respectively. The prevalence of senile dementia in the elderly with non-education was higher. The prevalence of senile dementia in women was higher than that in men. The prevalence of senile dementia increased consistently with advancing age. The age of patient affected VD was younger than that of $\mathrm{AD}$. The prevalence of $\mathrm{AD}$ between the urban and the rural areas had no significant difference. The prevalence of senile dementia and AD in Chengdu cohort is similar to that from Shanghai and other countries. The prevalence of senile dementia increases with age advancing and is higher among the non-educated elderly.

89

A Preliminary study of the epidemiology of successful aging in community elderly

C.B. $\mathrm{Li}^{1}$, M.Y. Zhang ${ }^{1}$, Y.L. He ${ }^{2}$, et al. ${ }^{1}$ Tongji Hospital of Tongji University, Shanghai 200065, P.R. China; ${ }^{2}$ Shanghai Mental Health Center, Shanghai 200030, P.R. China

This study is to investigate the prevalence of usual aging, successful aging and mild cognitive impairment in community elderly and explore the related factors of successful aging, a cross-sectional survey was conducted in this study. The sample was the elderly who live in community and aged 65 or over. The sampling site was two streets in Putuo District, Shanghai. The instruments included demographic contents, some international standardized instruments (such as MMSE, ADL, SF-36, LSIA, DUFSS, etc.). The results showed that, among the 1516 elderly interviewed, the prevalence of successful aging, usual aging and mild cognitive impairment (MCI) groups in the large sample cross-sectional study with 1516 individuals living in community aged 65 or above had were $46.17 \%$ (95\% CI: $43.66 \%-48.68 \%$ ), $40.11 \%$ (95\% CI: $37.64 \%-42.57 \%$ ) and $9.70 \%$ (95\% CI: $8.21 \%-11.19 \%$ ) respectively. Some special psychosocial factors were found related to successful aging, the quality of life of successful aging was higher than that of usual aging or MCI. Our results suggest that successful aging has a multi-dimensional characteristic among the community elderly and the related factors of successful aging may provide theory and strategy for efficient intervention.

90

Bibliometric Analysis of Parkinson disease

H.G. Li, S.G. Sun, E.T. Tong, et al. Department of Neurology, Union Hospital, Tongji Medical College, Huazhong University of Science and Technology, Wuhan 430022, P.R. China

To investigate the research status and trends of Parkinson disease (PD), we analyze the data source from CBMDisc and MEDLIN database and classified by headings, the numbers of the articles on PD research between 1990 and 2000 were analyzed by bibliometrics. It was found that the number of articles on PD research was increasing years by years, not only in foreign country, but also in China. Among the 15 research foci on PD in recent 5 years, pharmacological therapy was in the first place. Chinese traditional medicine has taken a large portion of the whole PD therapy research in China. The result indicates that 
the research on PD has great prospect. The focus on PD research in China was almost similar with that in foreign country in recent five years, but several of research foci in China was relative lagged. The main PD research subject in China was different methods of therapy, among them Chinese traditional medicine has taken an important place.

91

The Prevalence of Parkinson's disease in the Nutritionally Deficient Population of Linxian, P.R. China

Z.Y. Nie ${ }^{1}$, L. Zhang ${ }^{2}$, Ying Liu ${ }^{1}$, et al. ${ }^{1}$ The Second Affiliated Hospital of Dalian Medical University, Dalian, P.R. China

The aim of this study is to determine the prevalence of idiopathic PD in the nutritionally deficient population of Linxian Country for evaluating the long term effects of nutritional intervention on PD and making recommendations of prevention, a cross sectional study within a cohort of adults over 50 years old who participated in a nutritional intervention trial between 1985 and 1991 was conducted. All participants were screened by questionnaire for parkinsonism and general neurological examination by junior neurologists. The crude prevalence rate of PD was 522/100,000 among subjects 50 years old or over. Assuming no case of PD among individuals under 50 years old, the age-adjusted prevalence rate standardized to the age composition of the 1990 census in P.R. China was 77.66/100,000. The age-specific prevalence of PD was significantly higher than that in other areas in P.R. China. The age-adjusted prevalence of PD was 6 or more times higher than that in other areas. These findings suggested that chronic nutritional deficiency may be one of the risk factors for $\mathrm{PD}$

92

Association between Angiotensin-converting Enzyme Gene and Late Onset Alzheimer's disease in Han Chinese

C.S. Wu, D.F. Zhou, Z.Q. Guan. Institute of Mental Health, Peking University, Peking 100083, P.R. China

As a major player of vascular incidents, ACE gene may be a candidate gene. A case-control study in $\mathrm{AD}$ group including subjects with hypertension or normal blood pressure, as well as in control group, was conducted. The difference of ACE gene deletion (D)/ insertion (I) polymorphism was compared. Also the ApoE genotyping to investigate subjects with ACE I/D allele and E4 allele on the risk of $\mathrm{AD}$ was examed, as well the difference of age between $\mathrm{AD}$ with these alleles and without them. The result presented that there was no significance between cases and controls in genotyping and in I/D allele distribution. But as far as hypertension was concerned, AD patients and controls with normal blood pressure, there was significant difference in ACE genotypes and alleles distribution. And there was no significance on I/D distribution in ApoE every genotype between $\mathrm{AD}$ cases and controls. Our results suggest the ACE polymorphism association with the risk of $\mathrm{AD}$ is still be elucidated. The hypertension maybe involves in the ACE I/D different distribution in AD.

Analysis of Cognitive Level and Ability of Daily Life in Above Aged 55 Population, Alzheimer's and Parkinson's Disease and in Linxian Country of Henan Province, P.R. China

D.F. Zhou, C.S. Wu, C.S. Wang, et al. Institute of Mental Health, Peking University, Peking 100083, P.R. China

The objective is to investigate the impairment in cognitive level and ability of daily life on AD, PD and above aged 55 population in the four communes in Linxian Country, Henan Province, P.R. China, a cross sectional study was conducted within a group of adults older than 55 who participated in a nutritional intervention trial between 1984 and 1991 in Linxian. All subjects were screened using the Chinese version of the MMSE (CMMS), ability of daily life (ADL) and PD questionnaire. Among 16488 subjects interviewed, 16095 subjects completed CMMS. There was significant difference between CMMS score of male $(24.71 \pm 3.30)$ and that of female $(22.17 \pm 3.68)$ $(P<0.001)$. There were also significant difference in CMMS total score and its every factor among groups of educational year $<1$, educational year $1-6$ and educational year $\geqslant 7$. Also CMMS score between plain population $(23.81 \pm 3.62)$ and mountain population group $(23.29 \pm 3.92)$ was significant $(P<0.001)$. The CMMS score $14.49 \pm 3.72$ in AD group, and $21.67 \pm 4.23$ in PD group and $23.76 \pm 3.55$ in above aged 55 control group were significantly different $(p<0.01)$. As analysis for the 7 factors of CMMS, there were significant differences in all seven factors in $\mathrm{AD}$ group compared with PD group and with above aged 55 control group $(p<0.001)$. Comparing PD with control, Orientation of Time, Orientation of Place, Ability of Calculation, Short-term Memory were significantly higher than that in control group $(p<0.01)$, and Immediate Memory was higher too $(p<0.05)$. The ADL score in AD group was $17.91 \pm 8.97,14.58 \pm 4.94$ in PD group and $13.41 \pm 2.12$ in control group. And it was significant difference between $\mathrm{AD}$ and $\mathrm{PD}$ group $(p<0.001)$, AD and control group $(p<0.001)$, PD and control group $(p<0.01)$. ADL score between group of normal CMMS $(13.34 \pm 1.74)$ and group of abnormal CMMS $(15.83 \pm 6.85)$ was significant difference $(P<0.001)$. Every ADL item score increased in AD group, but only Using Public Transportation, Walking, Cooking and Housing increased in PD group. We concluded that the score of CMMS is decreased with the aging of population and lower educational level. Both AD and PD patients have cognitive dysfunction, but cognitive impairment in $\mathrm{AD}$ and $\mathrm{PD}$ has their own characteristic, and cognitive deficiency in $\mathrm{AD}$ was severer than that in PD. The impairment of ADL in AD patients is more extensive than that in patients with $\mathrm{PD}$

94

An Association Study of the Polymorphisms in Apolipoprotein E Gene and Senile Cardiovascular Disease

Q.P. Wu, P.Y. Li, S.Q. Wu, et al. First Hospital of Sanming, Fujian 365000, P.R. China

The study was conducted to investigate the relationship between senile cardiovascular disease and the polymorphisms of apolipoprotein E (ApoE) gene. Both case-control association analysis and polymerase chain reactionrestriction fragment length polymorphism (PCR- RFLP) technique were used to detect the genotypes of ApoE gene in 351 patients with senile cardiovascular disease and 103 healthy controls. Prominent difference between cases and control was observed in all allele distributions of ApoE gene, $\varepsilon 2$ and $\varepsilon 4$ frequencies higher and $\varepsilon 3$ frequency lower in cases. By association analysis, it showed a positive correlation between $\varepsilon 2$ and $\varepsilon 4$ alleles and the disease, then $\varepsilon 3$ showed a negative correlation with the disease. It was found that the $\varepsilon 2$ and $\varepsilon 4$ alleles of ApoE gene may be the impressibility factors of senile cardiovascular disease, then $\varepsilon 3$ has an opposite effect on the disease.

95

A nested case-control study of Parkinson's disease in a nutrition intervention cohort

H. Xiao ${ }^{1}$, L. Zhang ${ }^{2}$, W. Chen ${ }^{3}$, et al. ${ }^{1}$ Dalian Medical University, Dalian, P.R. China; ${ }^{2}$ University of Rochester, New York, USA; ${ }^{3}$ Cancer Institute Chinese Academy of Medical Science, Beijing, P.R. China

The purpose of this study was to identify potential risk factors for Parkinson's Disease(PD) in a nutritionally deficient population in Linxian of P.R. China. A nested case control study was conducted within a cohort of 16488 rural adults aged 55-89 years who participated in a randomized nutrition intervention trail (NIT) in 1986. Cases $(n=8)$ were diagnosed using a two phases screening by neurologists and interviewers by May 2000. Controls randomly selected from the same cohort, were matched (control/case ratio $=4$ ) for sex, age $(+/-5$ years) and residential. Consumption of eggs, fruits mouldy food, mile chaff, pickled vegetable were similar between case and control subjects. Consumption of meat reveals inverse effects. Risk significantly declined as pre-trial body mass index (BMI) increased. The study found that a history of gastric ulcer was associated with elevated risk for PD. Different to the results of some other of PD with smoking. These results indicated that smoking, consumption of meat) are inversely associated with $\mathrm{PD}$, which supports a role for environmental factors in the etiology of PD.

Study of senile dementia epidemiology of old-aged population in urban area with high incidence of cerebrovascular disease

Q.D. Yang, Y.H. Zhou, X.L. Tang. Institute of Neurology, Xiangya Hospitol, Central Southern University, P.R. China

The purpose of this study was to investigate the senile dementia epidemic of community population in urban area with high incidence of cerebrovascular disease. A survey of senile dementia was carried out in community population in Changsa urban from July 1st, 2000 to June 31th, 2001. The investigation found that the prevanlence ratios of dementia were $2.88 \%$ in the population aged 60 years and older, $1.51 \%$ for Alzheimer's disease, $0.89 \%$ for vascular dementia and $0.48 \%$ for mixed dementia. And the percentages of hypertension and stroke history were significant higher in dementia than that in all population surveyed. The results indicated that senile dementia was an important factor affecting body and mind health of old-aged population and to prevent the cerebrovascular disease must be postive effect of discreasing the prevalence ratios of dementia.

The Correlation between Cognitive Function Impairment and Allele Geno-type of ApoE among 354 Apparently Healthy ElderLy in Shanghai, P.R. China 
Y.X. Ma, P.F. Lu, Y. Zhu, et al. Department of Aging, Antiaging and Cognitive Function, Shanghai Institute of Gerontology and Geriatrics, Huadong Hospital, P.R. China

The allele genotype of ApoE and cognitive function (WMS, MMSE, HDSR) of 354 apparently healthy elderly (aged $65-85$ yrs) in Shanghai were determined in order to evaluate if there were correlation between genotype of ApoE and cognitive function impairment. The results (score) were as following: (1) ApoE $\varepsilon 3 / 3$ ( 254 persons): MQ $94.0 \pm 16.9$, MMSE $28.3 \pm 2.2$; HDS-R $27.1 \pm 3.6$ (2) ApoE $\varepsilon 2 / 3$ (41 persons): MQ 91.1 \pm 19.0 , MMSE $27.5 \pm 2.8$; HDS-R 26.1 \pm 3.4 (3) ApoE $\varepsilon 3 / 4$ (52 persons): MQ 92.4 \pm 18.7 , MMSE $27.8 \pm 3.5$, HDS-R $27.1 \pm 3.9$ (4) ApoE $\varepsilon 2 / 4$ ( 2 persons): MQ $98.5 \pm 2.1$, MMSE 27. \pm 0 , HDS-R $23.5 \pm 6.4$ (5) ApoE $\varepsilon 2 / 2$ (2 persons): MQ $92.5 \pm 0.7$; MMSE $29.0 \pm 1.4$; HDS-R $26.5 \pm 0.7$ (6) ApoE $\varepsilon 4 / 4$ (3 persons): MQ $92.0 \pm 16.1$. No significant difference of the score of MQ, MMSE, HDS-R (F value equaling $0.285,0.934$ and 1.117 respectively) in various ApoE genotypes were found. It is suggested that the effect of APOE as a risk factor on $\mathrm{AD}$ may not necessarily mediate the equal effect on cognitive function impairment in the apparently healthy elderly.

98

Prevalence of dementia and major subtypes in four cities of northern and southern P.R. China

Z.X. Zhang, Q.M. Jue, Z. Hong, et al. Department of Neurology, Peking Union Medical College Hospital, Chinese Academy of Medical Sciences, Beijing, P.R. China

The research is to investigate prevalence and epidemiological pattern of dementia and major subtypes in the elderly residents of northern (Beijing, Xian) and southern (Shanghai, Chengdu) P.R. China. A door-to-door three-phase procedure was used to ascertain dementia. A total of 1283 cases of dementia (63.1\% for $\mathrm{AD}$, and $26.8 \%$ for $\mathrm{VD}$ ) were identified. Crude prevalence in persons aged $55+$ was $3.4 \%$ for overall dementia, $2.2 \%$ for $\mathrm{AD}$, and $0.9 \%$ for VD. The corresponding rates in persons aged $65+$ were $5.3 \%, 3.6 \%$ and $1.2 \%$. Age-standardized prevalence (adjusted to the 1998 USA population) in persons aged $55+$ was $4.6 \%$ for overall dementia, $3.2 \%$ for $\mathrm{AD}$, and $1.0 \%$ for $\mathrm{VD}$. The corresponding rates in persons aged $65+$ were $7.0 \%, 5.1 \%$ and $1.3 \%$. The prevalence of $\mathrm{AD}$ increased rapidly with age from $0.1 \%$ among those aged $55-$ 59 to $30.1 \%$ at age $90+$. The prevalence of VD increased from $0.6 \%$ in age $55-$ 59 to $2.1 \%$ at age $80-84$, then turned down. The age-standardized prevalence of $\mathrm{AD}$ in persons aged $55+$ for women was significantly higher than that for men, i.e., $3.9 \%$ versus $2.2 \%$. Nevertheless, the age-standardized prevalence of VD for women was preceded by that for men, but not significant $(0.9 \%$ Versus. $1.2 \%)$. Variation between northern and southern was great for VD prevalence, and less for $\mathrm{AD}$ prevalence. The difference in age-standardized prevalence of $\mathrm{AD}$ between urban and rural was not significant that was $3.6 \%$ versus $4.2 \%$ in northern, and $2.4 \%$ versus $3.5 \%$ in southern. The age-standardized prevalence of VD in rural communities $(1.9 \%)$ was significantly higher than that in urban communities $(1.3 \%)$ in northern; however, the figure $(0.4 \%)$ in rural was not higher than that $(0.8 \%)$ in urban in southern. There were no significantly associated between lower education attainment and higher ageand sex-standardized prevalence rates for $\mathrm{AD}$ and VD either in urban or rural area of northern and southern P.R. China. In conclusion, our overall prevalence of dementia and its subtypes is considerably higher than previously reported estimates from P.R. China and similar to those reported in whites. Compared to $\mathrm{VD}, \mathrm{AD}$ was more prevalent in P.R. China, either in northern or in southern, in urban or in rural, in men or in women. The overall similarity for AD in both the prevalence estimates and age, gender patterns across regions suggests that limited variation of main risk factors related to $\mathrm{AD}$ present worldwide. We did not find evidences to support that lower education and rural setting are independent risk factors for $\mathrm{AD}$.

99

Prospective Study on the Relationship Betweennosocomial Infections and Hypothyroidism in the Aged

P.F. Lu, Y.X. Ma, X.F. Yu. Department of Aging, Antiaging and Cognitive Function, Shanghai Institute of Gerontology and Geriatrics, Department of Geriatircs of Huadong ospital, 221 Yan An West Road, Shanghai 200040, P.R. China. Email: yangjiandong@hotmail.com

This paper is about prospective study on the relationship between nosocomial infections and hypothyroidism in the aged. Prospective follow-up of elderly ( $\geqslant 60$ years) patients for nosocomial infections (N.I.) in geriatric ward from Jan. 1996 to Dec. 1997 after thyroid function tests (TT3, FT3, TT4, FT4, TSH) in 1995 in the healthy inspection of the aged in O.P.D. The mean values of TT3, FT3, FT4 and TSH in 134 cases complicated with N.I. are significanlly lower than that in 114 cases without N.I. The relative proportions of N.I. in various levels of TT3, FT3, FT4 and TSH are significantly higher than that without N.I. Hypothyroidism is intimately correlated with N.I., and may be one of the important causes of N.I. in the aged. It is suggested that routine examination of thyroid function is very important for prophylactasis of N.I.

\section{Clinical features and Treatment}

\section{0}

\section{Treatment strategies in Alzheimer's disease}

B. Winblad, A. Nordberg, V. Jelic. Karolinska Institutet, Huddinge Hospital, Sweden

Cholinesterase inhibitors (ChEI) are currently the most established treatment strategy in Alzheimer's disease (AD). The treatment effect appears mainly to be symptomatic. Effects on progression of the disease and possible neuroprotective effects for the ChEI have been investigated. Delay of nursing home placement has been reported. Four ChEI are approved in Sweden, tacrine, donepezil, rivastigmine and galantamine. The ChEI's may interact with $\mathrm{A} \beta$ production.

Nicotinic agonists may have cytoprotective effects. Nicotinic agonists can prevent glutamate-induced cytotoxicity. $\mathrm{A} \beta$ toxicity in cell cultures can be attenuated by treatment with nicotine. These experimental data fit well as a treatment strategy with the clinical observations that in the brain of $\mathrm{AD}$ patient, loss of nicotinic receptors correlate with the impairment in cognitive function. Glutamatergic (NMDA)-antagonists (memantine) has recently been successfully tested in severe dementia.

Estrogen replacement therapy effect has been based on epidemiological data that indicated a lower relative risk for AD in elderly women who took estrogen. Experimental data have shown that estrogen stimulates several neurotransmitters including acetylcholine, activates nerve growth factors, increases cerebral blood flow and has a possible neuroprotective effect. However, the three preliminary reported three estrogens treatment studies on AD have been negative.

Anti-inflammatory drugs have also in cross-sectional studies been shown to reduce risk for $\mathrm{AD}$. To our knowledge no data on incidence of $\mathrm{AD}$ have been presented for patients taking anti-inflammatory drugs. Propentofylline, a xanthine derivative, affects the glial function with less activation of microglial cells and has shown to be effective in demented patients. Recent congress reports with anti-inflammatory drugs (including Cox-2 inhibitors) have been negative in $\mathrm{AD}$ trials.

Growth factors are important for neuronal development and maintenance. Nerve growth factor (NGF) stimulates outgrowth of cholinergic neurons and intraventricular administration of NGF to three AD patients resulted in an increase in nicotinic receptor binding as measured by PET. However, severe pain, anorexia and weight loss were side effects that ruled out further clinical trials with that dosage and way of administration. Newer drugs that could be taken orally and stimulate growth factor production will soon be tested.

Anti-amyloid substances might be used to target directly to the production of $\mathrm{A} \beta$ in order to increase the removal of $\mathrm{A} \beta$ and/or to decrease the aggregation of $\mathrm{A} \beta . \mathrm{A} \beta$ is deposited in the senile plaques after the amyloid precursor protein (APP) in the AD brain is cleaved abnormally by $\beta$ or $\gamma$ secretases. Recent immunisation studies of APP transgenic mice with A $\beta 42$ before the onset of pathology prevented development of $\beta$ amyloid plaques and when given to mice already with pathology the progression was reduced. This might open the way for immunisation/vaccination against AD. Clinical studies have started. Studies on humans have also been initiated with a $\gamma$-secretase inhibitor.

Management of Aging Patients with Parkinson's diseases Treated with Microelectrode-guided Stereotactic Pallidotomy and Thalamotomy G.Y. Cui, H. Feng, X.R. Wang, et al. Department of Neurosurgery, Southwest Hospital, Third Military Medical University, Chongqing 400038, P.R. China

The aim of this research study treatment methods and outcome of 28 aging patients with PD treated with microelectrode-guided stereotactic pallidotomy and thalamotomy, the localization of target is achieved by both MRI and CT scans. Pallidal and thalamal target sites are chosen by supervision of microelectrode recording technique in 28 aging patients above 70 years old with PD. 2 type-A patients underwent unilateral vertrolateral thalamotomy, and 1 contemporary bilateral ventrolateral thalamotomy and pallidotomy. 26 patients underwent unilateral posteroventral pallidotomy, 1 contemporary unilateral ventrolateral thalamotomy and posteroventral pallidotomy. Preoperatively, the UPDRS motor score was identified, and followed up 12 months interval. After 12 months follow-up monitoring, tremor was resolved completely or nearly completely in 2 patients who underwent unilateral and 1 of them bilateral ventrolateral thalamotomy and pallidotomy, and mandible tremor was improved. Dramatic improvement of tremor, rigidity, bradykinesia was observed in 26 pa- 
tients undergoing posteroventral pallidotomy. Microelectrode-guided stereotactic pallidotomy and thalamotomy are effective measures for treating $\mathrm{PD}$, but the indication should be controlled seriously.

102

Clinic Study on Early Poststroke Pepression

H. Hong, R.X. Huang, T.H. Wang, et al. Department of Neurology, The First Affiliated Hospital, Sun Yan-Sen University of Medical Sciences, Guangzhou 510080, P.R. China

80 cases who had only single stroke focus were selected from the acute stroke patients (excluding SAH) and were examined with the Hamilton Depression Scale. The correlation between the incidence of poststroke depression (PSD), the manifestation, the feature of lesion and the regional blood flow were discussed combing with the CT scan and SPECT. Meanwhile, the affections of PSD on the recovery of the neurological impairment were analyzed through the assessment of the neurological function of the patients at various states of the treatment. In this study, the incidence of the early PSD was $32.5 \%$. The feature and the severity of PSD were related to the location of lesion and the regional blood flow. The presence of the early PSD might arise negative affection obviously on the recovery of the neurological impairment of the patients.

103

Clinical Observation on Intelligent Level of Vascular Dementia Treated with Acupuncture

J. Li, L.P. Lai, P. Li, et al. First Teaching Hospital of Tianjin College of T.C.M., Tianjin 300193, P.R. China

This study is to probe the mechanism of acupuncture involved in Vascular Dementia (VD) and clinical curative effect of "Reinforcing kidney, increasing illtelligence, calming the liver, clearing and activating collaterals", We selected 188 cases of VD patients, and tested their nervous psychology. In acupuncture group, Renzhong, Neiguan, Fengchi, Taichong, Baihui and Sishencong acupoints were selected. Treatment was conducted twice a day, two months in succession. Medicine group was treated with Duxil. At the same time Deng Zhan Hua Su Tablet was taken. In this study we adopted Wei Shi Adult Intelligence Measure Table and Hemorg Measure. Total effective rate is $73.44 \%$ in the acupuncture group, while that in medicine group is $51.67 \%$. There is significant difference in curative effect. Besides the remarkable difference in curative effect between two groups, there are also some other significant differences between them in VIQ, PIQ, FIQ and MQ. The level of Reinforcing kidney and Increasing Intelligence could effectively improve the level of intelligence and the obstructions of memory, correspondingly the brain function of the VD patients is improved. We should take protection activity against CVD to decrease the incidene of VD.

104

The clinical study of anxiety and depression disorders after stroke and transient ischemic attacks

F. Liu, P. Li. Neurological Department of Guiyang Medical College, P.R. China

The purpose of this study is to explore the occurring condition, influencing factors and correlative risk factors of anxiety and depression disorders after stroke and transient ischemic attacks. TIA group 76 cases and stroke group 70 cases, the diagnosis of both groups were confirmed by clinical manifestation CT and MRI. To adopt HAMD and HAMA scales to measure the anxiety state depression state of the patients of both groups. The anxiety and depression disorders were presented after TIA and stroke. There is no significant difference of incidence of anxiety state between both groups. There is significant difference of the incidence of depression state between both groups. The depression state in stroke group mainly expressed in anxious factor/somatization factor, cognitive disturbance factor, blocking factor, hopelessness factor. The incidence of anxiety and depression disorders was significantly higher in the patients associated with stroke risk factors. The anxiety and depression disorders are the common complications after TIA or stroke attack. It is very important for TIA or stroke patient and his or her family members to have the knowledge about the mental changes of anxiety and depression and to use the drugs to treat the anxiety and depression symptoms.

105

Epilepsy Accompanying Alzheimer disease

X.F. Wang, Y. Yan, S.Q. Wen, et al. Department of Neurology, the First Affiliated Hospital, Medical University, Chongqing 400016, P.R. China

The objective is to study the clinical characteristic of epilepsy in Alzheimer disease by investigating the types, time and antiepileptic efficacy of epilepsy accompanying AD. Thirteen patients with epilepsy accompanying Alzheimer disease were included in this study. 8 patients had presented with tonic-clonic or clonic or tonic seizures. One patient had simple partial seizures evolving to complex partial seizures and another had simple partial seizure with motor signs. One had Transient epileptic amnesia. Two with familial history of AD presented as myoclonus. Epileptic seizures is developing later in the disease. All of 13 patients have had 1-2 times of EEG examination and showed focal or multifocal spikes, sharp waves, with or without associated slow waves and paroxysmal generalized slow waves activity. 11 of the 13 epileptic patients with Alzheimer's disease are efficacy to antiepileptic drugs. The results suggest that epilepsy is a feature common to Alzheimer's disease. The main category of epileptic seizures is generalized tonic-conic seizures. Most of epileptic seizures accompanying AD are antiepileptic drugs-response.

106

Clinical Manifestation and Therapeutic Option of the Post-stroke Depression

J.H. Zhang, H.Y. Ren. Department of Neurology, People's Hospital, Liaocheng, Shandong, P.R. China

Psychological changes may occured in patients with cerebral vascular disease because of physiologic, social and economic factors. Main clinical manifestation of post-stroke depression consists of fear, despair, worry, et al. Atypical depressive symptoms such as dysphoria and anxiety is common. Treatment of post-stroke depression includes psychotherapy and anti-depression medicines.

107

Clinical trial of donepezil on patients with Alzheimer's disease

X.Q. Zhang, H.Q. Dong, B.Q. Min, et al. Department of neurology, Xuanwu hospital, Beijing, 100053 P.R. China

To observe the curative effect of donepezil on patients with Alzheimer's disease, $10 \mathrm{AD}$ patients were observed. The degree of cognitive impairment was mild-moderate. Donepezil $5 \mathrm{mg} /$ day was taken orally. 12 weeks were observed. MMSE, CDR and ADL were measured separately at 0, 6 and 12 weeks to evaluate the curative effect. Physical examination, laboratory test were used to assess the side effect. The grade of MMSE and ADL demonstrated that donepezil improved the function of cognition in patients with Alzheimer's disease. There was no changes of kidney and liver function caused by donepezil. Donepezil can improve the cognitive function of patients with Alzheimer's disease.

108

The Effect of SSRIs and Disorder of Platelet Serotonin on Elderly Depressive Symptom with Somatic Disease

K. Zheng, T.H. Shi, X.Q. Liu. Department of Neurology, Department of Internal Medicine, Tongji Hospital of Tongji Medical University, Wuhan, 430030 P.R. China

To discuss the effect of SSRIs and disorder of platelet serotonin on elderly depressive symptom with somatic disease. 30 cases of elderly depressive symptom were treated with SSRIs (paroxetine), all elderly patients were measured by ZUNG and HAMD scales and platelet 5-HT content before and after taking paroxetine. It was found that elderly patients had a higher level of platelet 5-HT content than normal ones. After six weeks of treatment of taking SSRIs (paroxatine), symptoms of depression, somato-symtom were significantly improved after therapy. It is suggested that SSRIs was an effective therapy in elderly depressive symptom with somatic disease.

\section{Diagnoses (Biochemistry, Behavioral tests and imaging)}

109

Informed Consent and Genetic Testing in Neurological Disorders - A Persisting Dilemma

R. Ravid. Netherlands Brain Bank, Amsterdam, The Netherlands

All international research codes require biomedical researchers to obtain the informed consent of subjects or their representatives before starting a study. This requirement poses special challenges and problems for investigators conducting dementia research. The cognitive capacities of the patients vary widely; individuals in the early stage of the disease can make their own choices whereas individuals with severe impairment will be incapable of making the informed choice.

The tremendous advances in genetic research raises serious ethical problems and complexities; very often there is conflicting interest in the need for knowledge and information on the one hand and the use and implications of this information for the providers, the families and the patients involved. The link 
found between certain genes and neurological diseases, creates a heavy burden on physicians, health care workers and researchers who test for these genetic factors as they are essential for the diagnostics and scientific interpretation of results in brain research of neurological diseases.

The most common cases of genetic testing we are confronted with in the Netherlands include the genes involved in Huntington's diseases, the Apo-E gene in $\mathrm{AD}$ and chromosome 17-Frontal temporal Dementia (17-FTD). The outcome of the genetic testing poses a heavy load on the concerned or afflicted individuals and their families.

Genetic testing has in some cases a very limited predictive value and there is a strong need for counselling combined with testing. Genetic testing can be psychologically incapacitating especially when at the moment there is no treatment or cure for most cases. The availability of the various tests for the public results in the need to know the differences between predictive testing and diagnostic testing. Early diagnostic tests may prove extremely useful when treatment becomes available which makes the information obtained by genetic testing essential for the scientific effort to develop the right therapeitic strategies.

Fluorescence Polarization Assay for Fibrillar Amyloid Beta-protein

V. Chauhan, A. Chauhan, J. Wegiel. NYS Institute for Basic Research in Developmental Disabilities, 1050 Forest Hill Road, Staten Island, NY 10314

Fibrillization of amyloid beta-protein $(\mathrm{A} \beta)$ in the brain is a key event in the pathogenesis of Alzheimer's disease. Several factors such as metal cations $-\mathrm{Zn}^{2+}, \mathrm{Al}^{3+}$ promote the in vitro fibrillization of synthetic soluble amyloid beta - protein, while other factors such as gelsolin, melatonin inhibit it. The fibrillization of $\mathrm{A} \beta$ is measured by Thioflavin $\mathrm{T}(\mathrm{ThT})$ fluorescence spectroscopy and Congo red (CR) absorbance spectroscopy, or by time consuming electron microscopy. Cations and colored factors often interfere with ThT and CR assays. Therefore, an assay is required that can measure the fibrillization of $\mathrm{A} \beta$ in the presence of such interfering factors. We report here that fibrillar $\mathrm{A} \beta$ forms membrane - like hydrophobic domain, and the formation of these domains can be measured by fluorescence polarization of 1,6 - diphenyl 1,3,5 - hexatriene (DPH) inserted in these domains. Soluble $\mathrm{A} \beta$ was not found to induce DPH fluorescence polarization. No interference of cations was observed when $\mathrm{A} \beta$ was fibrillized in the presence of cations. We have reported previously that gelsolin, an actin binding protein, inhibits the fibrillization of $\mathrm{A} \beta$. In DPH fluorescence polarization assay also, we observed the complete inhibition of $\mathrm{A} \beta$ hydrophobic domain formation in the presence of gelsolin. The formation of hydrophobic domain was not observed with the polymerized actin. These results suggest that DPH fluorescence polarization can be used as an assay to measure the fibrillar $\mathrm{A} \beta$.

\section{1}

Mirror Writing and Cerebral Function Gradation and Degradation

X.D. Wang. Department of Neurology, Beijing Hospital, Beijing 100730, P.R. China

This paper includes following topics: 1 . Methods of examination of mirror writing (MW) in Chinese characters. 2. The grading of the severity of MW, 3. MW in the children. 4. MW in healthy adults. 5. MW in the elderly. 6. MW in patients with nervous diseases (cerebral vascular diseases, Parkinson's disease, senile dementia and vascular dementia in the elderly patients). 7. Discussion on pathogenesis of MW.

\section{2}

The Research of Dopamine Transporter Imaging of Patients with Parkinson's Disease

S.Y. Bao, W.F. Luo, J.C. Wu, et al. Department of Neurology, Second Affiliated Hospital of, Suzhou University, P.R. China

The first purpose of this study was to evaluate the value of $99 \mathrm{~m} \mathrm{Tc}$ TRODAT $_{-1}$ SPECT DAT imaging for early diagnosing PD. Second, we studied the relationship between the severity of PD and the changes of DAT concentrations in striatum. Twenty three patients with PD and eighteen healthy

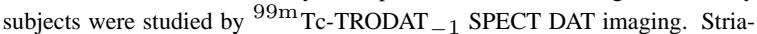

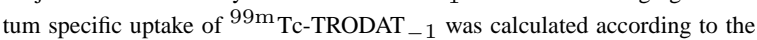
ratio of striatum (ST) and cerebellum $(\mathrm{CB})$. All patients with PD were scored on the UPDRS and Hoehn-Yahr stages. In the hemi-Parkinson's disease group, the DAT specific uptake of ${ }^{99 \mathrm{~m}_{\mathrm{Tc}} \text { TRODAT }}$ - 1 was significantly lower $(P<0.01)$ in contralateral $(1.32 \pm 0.03)$ than in ipsilateral striatum $(1.36 \pm 0.04)$ to the clinically symptomatic side. There was significant reduction $(P<0.01)$ of striatum DAT uptake in patients with hemi-PD (LST: $1.35 \pm 0.04$; RST: $1.33 \pm 0.04)$ compared to the age gender-matched controls (LST: $1.37 \pm 0.05$; RST: $1.36 \pm 0.04$ ). Significant negative correlations were found between scores of total UPDRS, activities of Daily Living, Motor examination and the ST/CB ratio of $99 \mathrm{~m}_{\mathrm{Tc}}$-TRODAT 1 uptake. There was not correlation between the ST/CB ratio and the score of Mentation Behavior and Mood. The results demonstrated that ${ }^{99 \mathrm{~m}_{\text {Tc-TRODAT }} 1 \text { SPECT DAT }}$ imaging can not only help us to confirm the diagnosis of PD at the early stage, but also assess the severity of PD.

113

\section{The Value of CSF A $\beta$ in Diagnosis of Alzheimer Disease}

X.S. Ding, H. Cheng, X.L. Zhang, et al. Nanjing Medical University, Nanjing 210029, P.R. China

Recently several studies demonstrated the senile plaques are association with $\beta$ amyloid protein $(\mathrm{A} \beta)$ which is a kind of polypeptides derived from $\beta$ protein precursor (APP) and also called $\beta \mathrm{A} 4$ (4KD molecule). In this article, the cerebrospinal fluid (CSF) levels of $\mathrm{A} \beta 1-40$ and $\mathrm{A} \beta 1-42$ in patients with $\mathrm{AD}$ were analyzed for studying the value of diagnosis on $\mathrm{AD}$. The CSF levels of $\mathrm{A} \beta 1-42$ in $78 \mathrm{AD}$ patients, 30 normal controls (NC) were analyzed by sensitive enzyme linked immunosorbant assays (ELISA); the CSF absorbance (A) levels of $\mathrm{A} \beta 1-40$ were also detected in 24 patients and $\mathrm{NC}$ group, and the ratio of $\mathrm{A} \beta 1-40$ to $\mathrm{A} \beta 1-42$ ( $\mathrm{A} \beta$ ratio) was calculated at the same time. The results suggested significantly decreased levels of CSF A $\beta 1-42$ in AD patients $(p<0.01)$. The level of CSF A $\beta 1-42$ absorbance values of $\mathrm{AD}$ group was decreased which was significantly different from the level of $\mathrm{NC}(p<0.05)$. No difference in $\mathrm{A} \beta 1-40$ absorbance levels was observed in the two groups. $\mathrm{A} \beta$ ratio was constant in $\mathrm{NC}$ group, but significantly increased in $\mathrm{AD}$ group $(p<$ 0.05 ). If using the $\mathrm{A} \beta 1-42$ concentration of $294.28 \mathrm{pg} / \mathrm{ml}$ and the $\mathrm{A} \beta$ ratio 10.4 as the cutoff value, the sensitivity in diagnosis of $\mathrm{AD}$ is $38.46 \%, 60.50 \%$, the specificity is $96.77 \%, 88.46 \%$ respectively. $\beta$ A 4 has neurotoxicity probably by its function as follows: medium of receptors, medium to activate microglial, activation and direct combination with soluble $\mathrm{A} \beta$, abnormal metabolism of $\mathrm{APP}$, or disturbed intracellular transport in neuron. $\mathrm{A} \beta 1-40$ and $\mathrm{A} \beta 1-42$ are major component of released $\mathrm{A} \beta$. $\mathrm{A} \beta 1-40$ is a constituent molecule of matured senile plaque and amyloid angiopathy. A $\beta 1-42$ is a highly amyloildogenic initial component of senile plaque amyloids. In our study, the constant ratio of $\mathrm{A} \beta$ ratio in $\mathrm{NC}$ group was elevated in $\mathrm{AD}$ patients, probably because of the alteration of recretase activity or clearence activity of distinct $\mathrm{A} \beta$ species, abnormally accumulation of $\mathrm{A} \beta 1-42$ in brain that might induce the decline of $\mathrm{CSF} A \beta 1-42$ in AD groups. The paper showed that the cutoff value of the $\mathrm{A} \beta 1-42$ concentration and $\mathrm{A} \beta$ ratio in NC group provide $38.46 \%, 60.50 \%$ sensitivity and $96.77 \%, 88.46 \%$ specificity respectively in diagnosis of AD.

114

The significance of atypical Parkinsonian symptoms and Abmormal Iron Deposittion on MRI in the Diagnosis of early stages of MSA

G.J. Wang. Department of Neurology, Renmin Hospital, Wuhan University, Wuhan 430060, P.R. China

In this study, the clinical data in 13 cases with clinical diagnosis MSA were reviewed retrospectively. Age of onset ranged from 42 to 68 (mean 61) years. Of 13 patients, 7 were diagnosed as MSA-OPCA, 4, MSA-SDS, 2 , MSA-SND. All but three (76.9\%) had an akinetic-rigid symdrome.The other clinical features included cerebellar ataxia in 7 cases, intention tremor in 3 , frequent syncopal episodes, dizziness on standing or orthostatic hypotension in 4 , urinary urgency,urinary frequency or incontinence in 4 , faecal incontinence in 1 , impotence or loss of ejaculation or declining sexual function in 4, hyperreflex or extensor plantar response in 2 , excessive snoring in 1 , early falling in 2 , hyperthemia in high temperature weather in summer and depression in 1 . None of these patients had a family history of neurological diseases.Definite diagnosis in these patients was made in only 9 cases who presented with severe autonomic dysfunction or slowly progressive cerebellar ataxia. In the remaining 4 cases, all were misdignised as PD in their early stages of diseases due to predominant parkinsonian symptoms. Retrospectively three of 4 had subtle atypicical parkinsonian symptoms and 2 had mild cerebellar atrophy or decreased T2 signal in the globus pallidus. These observations indicate that both increased abmormal iron deposittion on MRI and atypicical parkinsonian symptoms in the early stages of the disorders, including autonomic dysfunctions, cerebellar ataxia, absence of resting tremor, pyramidal tract signs, and failure to respond to levodopa may greatly contributed to a final diagnosis of MSA

115

Detection of Serum Apolipoprotein E levels in patients with Vascular Dementia and Cerebral Infarction

K. Zhou, F. Li, S.Z. Yu. Neurologic Department, Renmin Hospital of Wuhan University, P.R. China

To investigate apolipoprotein $\mathrm{E}(\mathrm{ApoE})$ role in the pathogenesis of vascular dementia (VD) and cerebral infarction (CI), we measured serum ApoE, TC, TG, HDL-C, LDL-C, ApoA, ApoB levels in 20 cases of VD, 24 cases of CI 
and 24 sex-and age-match control subjects by monoclonal anti-enzyme linked immunosorbent assay (McAb-ELISA). Higher serum ApoE levels was found in $\mathrm{VD}$ and $\mathrm{CI}$ groups $(\mathrm{P}<0.01)$, but no difference between them $(\mathrm{P}>0.05)$. A positive association of ApoE levels with TC, LDL-C, ApoB in VD and CI groups, and a negative one with HDL-C $(\mathrm{P}<0.01)$. In conclusion, this date indicate that ApoE plays an important role in the pathogenesis of $\mathrm{VD}$ and $\mathrm{CI}$, serum ApoE could be used as a key index in abnormal lipid metabolism, and its sensitivity and specificity is superior to the other regular lab indexes.

\section{6}

Study a suitable diagnostic criterion of Parkinson's disease for screening in population

Z.Y. $\mathrm{Nie}^{1}$, L. Zhang ${ }^{2}$, X.D. Sun ${ }^{3}$, et al. ${ }^{1}$ The Second Affiliated Hospital of Dalian Medical University, Dalian, P.R. China, 116023; ${ }^{2}$ University of Rochester, New York, USA; ${ }^{3}$ Cancer Institute Chinese Academy of Medical Science, Beijing 100000, P.R. China

Parkinson's disease (PD) is a chronic neurodegeneration illness that affects a significant number of the older population. In this study, a cross sectional study was conduct to estimate the diagnostic criteria of PD and discuss a suitable criterion for screening in population. All participants were screened by questionnaire for Parkinsonism and general neurological examination by junior neurologists. If either was positive, the subject was examined by a senior neurologist using the United Kingdom Parkinson Disease Society Brain Bank Diagnostic Criteria (UKPDSBBDC) and other 5 criteria, respectively. The sensitivity and specificity of these criteria were discussed by regarding the UKPDSBBCDC as a golden criterion. These criteria were based on the combination of the main symptoms of PD such as bradykinesia (B), rigidity (R), resting tremor $(\mathrm{T})$, posture reflex abnormity $(\mathrm{P})$ and asymmetrical. Among the total 16,488 subjects screened in this survey, according to the UKPDSBBCDC, 86 patients were diagnosed as PD. The crude prevalence rate was $522 / 100,000$ among subjects 50 years of age or older. Assuming no case of PD among individuals below 50 years of age, the age-adjusted prevalence rate standardized to the age composition of the 1990 census in P.R. China was 77.66/100,000. The difference of prevalence rates according to the criteria $(B+R+T),(3$ of $B$, $\mathrm{R}, \mathrm{T}, \mathrm{P})$ and UKPDSBBCDC were not statistically significant. The specificity of $(\mathrm{B}+\mathrm{R}+\mathrm{T}),(3$ of $\mathrm{B}, \mathrm{R}, \mathrm{T}, \mathrm{P})$ is $96.4 \%, 95.1 \%$, and the sensitivity is $58 \%$, $58.2 \%$. The specificity of the other criteria is $84.5 \%, 85.1 \%$, and the sensitivity is $98.9 \%, 100 \%$. Our results suggest that the variation of the prevalence rates of PD was primarily attributed to the difference of diagnostic criteria. The sensitivity and specificity of the other criteria were not high. Since there was not an easy criterion now, UKPDSBBCDC is a suitable criterion for PD screening in population although the UKPDSBBCDC is complex in practice.

\section{7}

Development of An Ultrasensitive ELISA-Dienzyme Substrate Recycle Assay

Y.Y. Hu, S.S. He, J.Z. Wang. Tongji Medical College, Huzhong University of Science and Technology, Wuhan 430030, P.R. China

On the basis of conventional enzyme-linked immunosorbent assay (ELISA) and dienzyme substrate recycle, we have developed a highly specific and ultrasensitive biochemical assay, named ELISA-dienzyme substrate recycle. The detective range of conventional ELISA for measuring recombinant tau and abnormally phosphorylated tau purified from Alzheimer disease brain was $1 \mathrm{ng}$ to $32 \mathrm{ng}$. It was $0.75 \mathrm{pg}$ to $200 \mathrm{pg}$ and $0.5 \mathrm{pg}$ to $50 \mathrm{pg}$ by ELISA-dienzyme substrate recycle. Compared with the conventional method, the sensitivity of the technique established in the present study increased 400 and 1300 times, with a enlarged detective range of 8.5 and 10 times, respectively. It could detect abnormally phosphorylated tau in cerebrospinal fluid of Alzheimer disease and used effectively for the diagnosis of the disease.

\section{8}

The ratios of $\beta$ amyloid precursor protein isoforms in platelets from senile dementia patients and its significance of diagnosis

X.L. $\operatorname{Tan}^{1}$, W.W. Dong ${ }^{2}$, G.Y. LI ${ }^{2}$. ${ }^{1}$ Department of Neurology, Xiangya Hospital, Zhongnan University, Changsha 410008; ${ }^{2}$ Department of Neurology, the First Affiliated Hospital, Chongqing University of Medical Science, Chongqing 400016, P.R. China

To detect the expression of $\beta$ amyloid precursor protein (APP) in the elements of peripheral whole blood, analyze the platelet APP isoforms and their levels and study whether a differential level of platelet APP isoforms is specifically related to Alzheimer's disease (AD) and whether it can be considered a peripherally diagnostic index, the expression of APP in peripheral blood cells was determined by immunohistochemical analysis, and the levels of APP isoforms were evaluated by means of western blot analysis in platelets from 20 patients with $\mathrm{AD}$ (10 mild, 6 moderate and 4 severe), 10 patients with non-Alzheimer's-type dementia (NATD), 15 patients with non-dementia of other neurological disease (NDND) and 15 normal controls (NC). We found: (1) In peripheral whole blood, platelets were APP strong immunoreactivity, lymphocytes were APP weak immunoreactivity, and other whole blood cellular elements were not immunoreactivity; (2) Five APP isoforms (90 kd, 95 kd, $100 \sim 110 \mathrm{kd}, 120 \sim 130 \mathrm{kd}$ and $140 \sim 150 \mathrm{kd}$ ) are present in platelet and the molecular weight ranges from $90 \mathrm{kd}$ to $150 \mathrm{kd}$; (3) The ratios of the intensity of the 120-to-130 kd APP isoform to the 140-to-150 kd APP isoform, and the 120-to-130 kd APP isoform to the 100 -to-110 kd APP isoform were significantly lower in the $\mathrm{AD}$ and mild $\mathrm{AD}$ groups $(P<0.05)$, as compared with NC, NDND and NATD groups; (4) Both ratios of platelet APP isoforms positively correlated with the scores of neuropsychological tests in $\mathrm{AD}$ group $(r=0.464$ to $0.603, P<0.05$ or 0.01$)$, and negatively correlated with age in NC, NDND and NATD groups $(r=-0.407$ to $-0.614, P<0.05$ or 0.01) Our results suggest: (1) Platelet is the major source of circulating APP and $\mathrm{A} \beta$; (2) The lower ratios of platelet APP isoforms can be considered an early marker for $\mathrm{AD}$, allowing for discrimination between $\mathrm{AD}$ and other types of dementia.

119

The study of the diagnosis of Alzheimer's disease using 18F-FDG PET

L.J. Wang ${ }^{1}$, Z.P. Zheng ${ }^{1}$, A.W. Tang ${ }^{2}$, et al. ${ }^{1}$ Department of Neurology, Guangdong Provincial People's Hospital, Guangzhou 510080; ${ }^{2}$ The research center of PET, Guangdong Provincial People's Hospital, P.R. China

In order to study glucose metabolism of the brain in Alzheimer's disease (AD) by positron emission tomography (PET) and to investigate the imaging characteristics and diagnosis criteria of ${ }^{18}$ F-FDG PET in Chinese AD patients, 13 AD patients, 13 non-AD dementia patients including Parkinson's disease with dementia (PDD), vascular dementia (VD) and AD-VD, and 13 age-matched healthy subjects were accepted brain scan using Siemens ECAT EXEACT HR + scanner. The transaxial, coronal and sagittal images were reconstructed by computer. Semiquantitative analysis was applied to assess the metabolic function of brain by the ration of mean radioactivity of cerebral lobe to cerebellum $(\mathrm{Rcl} / \mathrm{cb})$. PET scan was compared with magnetic resonance imaging (MRI). We found that the abnormal imaging of $\mathrm{AD}$ patients was PET $100 \%$, MRI 76.92\%. The imaging characteristics of PET in AD patients showed three patterns: (1) bilateral parietal,temporal,hippocampus and frontal or occipital lobe symmetrical hypometabolism was $69.23 \%$; (2) bilateral temporal and hippocampus or frontal lobe symmetrical hypometabolism was $23.08 \%$; (3) bilateral parietal symmetrical hypometabolism was $7.69 \%$. The PET imaging of non-AD dementia showed that asymmetrical and multiple foci with decreased radioactivity and hypometabolism was $92.31 \%$. The areas of hypometabolism were in nigro-striatum system,thalamus and cerebral. The abnormal MRI imaging in non-AD dementia was $84.61 \%$. The results indicate that, excluding cerebral structural lesions in brain with MRI, bilateral or symmetrical cerebral hypometablism found in FDG PET can be considered as the special characteristics and diagnosis criteria of AD. It is very important for the early diagnosis of $\mathrm{AD}$ and different from non-AD dementia.

120

A control study of pathology and imaging of senile dementia of the Alzheimer type

Y.Zhang, J. Wu, M. Shu. Department of Neurology, The First Clinical Hospital of Norman Bethune University of Medical Sciences, Changchun 130021, P.R. China

The purpose of this study is to explore the correlation of pathological changes and imaging through the control study of pathology and imaging of 4 cases of senile dementia of the Alzheimer type (SDAT). Including HE, Gallyas silver dyeing, and four immunohistochemical methods were used in the pathological dyeing. The loss of neurons cells of all lobes of cerebral cortex, cingulate gyrus, Amygdaloid nucleus, hippocampal gyrus, parahippocampal gyrus, meynert basal nucleus, substantia nigra, locus ceruleus, dorsal nucleus of vagus nerve, senile plaques (SP) and the changes of neurofibrillary tangles (NFT) were observed in detail. The control study of pathology and imaging was carried out by comparison of CT of different phases before death and after death of SDAT. The degeneration of temporal lobe is marked: (1) the degeneration of middle frontal gyrus of cortex of frontal lobe is the most evident; (2) the degeneration of long gyrus is more evident than that of short gyrus as the cortex of insular lobe concerned (3) the degeneration posterior part of cingulated gyrus is more evident than that of anterior part. The pathological findings of SDAT mentioned above are basically in accord with the changes of imaging. 
intelligence research

L.D. Wen. Department of Neurology, Central Hospital of Wuhan, Wuhan 430014, P.R. China

Presently, the Wechsler Adult intelligence scale and MMSE up to DSM-IV standard are used in the valuation of the dementia level in and abroad the country. The method is cockamamie, affected by multi factors, inaccurately, absent of practicable measure standard. By the development of the electrophysiology of the brain, the change on the electrocortical potential induced by intellection has been step by step recognized. This research was inspired and improved by the research of the electrocortical potential at resting (non-intellection) period conducted by Japanese scholar. The highest potential is located on the extracranial $\mathrm{Cz}$ spot (central part of the parietal lobe), the summation of the potential reflects the change of the electrical activity of brain, E (summation of electrocortical potential $)=\mathrm{SP}($ stabilization potential $)+\mathrm{SCP}($ slow-wave change potential) $+<1 \mathrm{~Hz} \delta$ wave, but SP isn't influenced by the intellection and outside environment, $<1 \mathrm{~Hz} \delta$ potential can stabilize in the extent of $10 \sim$ $20 \mathrm{uV}$ at resting period. We validated this result in 100 normal individuals. The 8-lead electroencephalogram's amplifier and display were applied, the lead was meliorated into monopole, a wave filter was installed, the summation of selective $<1 \mathrm{~Hz} \delta$ wave potential was made. While intellection potential was induced by "digital repetition", we calculated the absolute value of the electric potential difference. Comparing 100 normal individuals with 68 vascular dementia patients before and after the treatment, we found that the absolute value of the electric potential difference in normal individuals $>50 \sim 120 \mathrm{uV}$ mild dementia $>50 \mathrm{uV}$; moderate dementia $30 \sim 50 \mathrm{uV}$; serious dementia $<20 \mathrm{uV}$,the difference was significant. The result can be applied in the diagnosis, effect valuation, prognosis tracing of the elderly dementia, it has such features as advancing, scientific and practical. At the same time, it has provided a new way to research the normal individual's intelligence.

122

Vascular Dementia and Alzheimer Dementia by Transcranial Doppler sonography

C.Y. Li, T. Li, Z.C. Hui. Department of Neurology, Renmin Hospital of Wuhan University, Wuhan, P.R. China

Fifty patients with multi-infarct dementia (MID), 30 with Alzheimer dementia (AD) and 30 health subjects were investigated by TCD. Patients with MID had lower mean and diastolic blood flow velocity and effective pulsative range (EPR), but higher pulsative index than the control group. When $A D$ were compared with MID, no significant difference was observed except EPR. By using receiver operating characteristic analysis (ROC), a cutoff point for EPR value of $-1 \mathrm{~cm} / \mathrm{s}$ was established, which provided an additional criteria for differentiation.

\section{Psychology and Gerontology health cares}

123

The Reveal Study: A Research Protocol for Susceptibility Genotyping, Risk Assessment and Counseling for Alzheimer's disease

T.C. Brown, N. Relkin, P.J. Whitehouse, L.A. Farrer, L.A. Cupples, S. Post, A.D. Sadovnick, S. LaRusse, J.G. Davis, K.A. Quaid, M. Barber, R.C. Green

Genes and other markers for complex diseases are rapidly being identified and presymptomatic risk estimates are now possible. The REVEAL Study (Risk Evaluation and Education for Alzheimer's disease) is an NIH funded project (R.C. Green, PI) to examine the impact of providing risk assessment, including Apolipoprotein E (APOE) genotyping, for Alzheimer's disease (AD). REVEAL investigators are using the well-established genetic epidemiology of the disease, including the risk attributable to different APOE polymorphisms, to develop risk profiles. Asymptomatic adult children of AD patients are randomized into one arm where risk assessment is based upon age, gender and APOE genotype, or a control arm in which risk assessment is based only upon age and gender. Trained genetic counselors communicate these risks (including APOE disclosure) and follow subjects carefully to determine the psychological and practical impact of this information. Outcome variables include measures of anxiety, depression and satisfaction with the risk assessment and counseling protocol, as well as real-world decisions to change retirement planning or insurance coverage. REVEAL is the first study to explore susceptibility genotyping, risk assessment and counseling in an age-related degenerative disease. For this reason, preparation for REVEAL involved collection of pilot data, the establishment of internal and external review boards and the implementation of extensive safeguards, including a Certificate of Confidentiality. Results will inform policy debates and future guidelines about the advisability of such disclosures as the human genome is decoded and the scope of risk assessment expands. We will present our approach to the development of research in this controversial area. Preliminary data will also be discussed.

124

Characteristic in the Clinic of Senile Patients with Depression in Parkinson's disease

Z.B. Chen. The Affiliated Hospital, Hainan Medical College, Haikou 570102, P.R. China

We studied the characteristic and CT image of the senile patients with depression in Parkinson's disease. 31 cases ( $>60$ years) of depression degrees in Parkinson's disease were graded by Hamilton rating scale for depression (HRSD). 27 of 31 cases were examined by CT scans. Different degrees of depression of the patients with Parkinson's disease were analyzed and compared by clinic and CT image. There were 16 cases with light degree of depression, middle 8 cases and severe 7 cases. The degrees of depression had relations with clinical types but without age, sex, course and stages. Moreover, the degrees of depression of rigidity types were more severe than tremble types in the Parkinson patients with depression. The rate of abnormal CT image was $74 \%$. The cases with enlargement of III ventricle and leukoaraiosis in middle and severe degrees of depression were more than ones in light degrees of depression. There were some damages of cerebral function and under cortical construction in the patients with depression in Parkinson's disease.

125

A clinical analysis of depression disorder in elderly patients in general hospital

X.Y. Chen, Department of neurology, Wenzhou No. 2 Hospital, Wenzhou 325000, P.R. China

The purpose of this study is to investigate the clinical characteristics and related factors of elderly patients' depression disorder in general hospital's neurological department. Totally 116 cases patients [69 male and 47 female, all more than 60 years old and average age was $(67.1 \pm 6.2)$ years] were matched the depression diagnostic criteria of CCMD-2-R and the standard score of Zung's depression self-measuring scale (SDS) were equal or more than 50. Their clinical manifestations and related factors were analyzed. Dyssomnia and fatigue acted as chief complaints accounted for $90 \%$ and $50 \%$ respectively. Headache, dizziness, myosalgia, ostealgia and paresthesia were also prominent body symptoms. Other clinical findings included palpitation, panting, gastrointestinal malaise, anorexia, loss of body weight, anxiety, easy to lost temper, interesting decreased, lack of pleasant sensation, avoidance of social intercourse, cognition and competence decreased and feeling of despair. The average total rough score of SDS was $50.64 \pm 8.35$. The etiology of depression disorder in our study was mainly secondary factors that accounted for $93.1 \%$. Among them, stroke, coronary heart disease, Parkinson's disease, hypertension, chronic stomach disorder and malignant tumor accounted for $27.58 \%$ (32/116), 16.38 (19/116), 14.65\% (17/116), $13.79 \%$ (16/116), 11.2\% $(13 / 116)$ and $9.48 \%(11 / 116)$ respectively, while psychological factors only accounted for $6.9 \%$ which were due to living occurrence $(2.58 \%, 3 / 116)$, living alone $(2.58 \%, 3 / 116)$ and retire $(1.72 \%, 2 / 116)$ respectively. The symptoms of mild depression patients were improved after treated by SSRI drugs for 4 weeks, while the symptoms of moderate or severe depression patients were improved after treated by tricyclic anti-depression drugs for 2 weeks. The average score of the two groups was $32.24 \pm 4.61$. The study demonstrated that tricyclic anti-depression drugs were more effective than SSRI, but the adverse effects were also more common relatively. The diagnosis of depression disorder in elderly patients in general hospital was easily to be missed because of the chief complaints were mostly body symptoms and the etiology of depression disorder in elderly patients was mostly secondary to primary body diseases, so the differentiation was important. SSRI drugs should be used as first choice and psychological therapy and controlling of primary body diseases were also necessary.

Life quality analysis and mursing strategy for the patient with senile dementia

C.Y. Liu, H.F. Zhu. Department of Neurology, Ren min Hospital of Wuhan University, Wuhan 430060, P.R. China

The biological basis of senile dementia is cerebral atrophy and degeneration or dysfunction of the brain cells. The main nursing problems and related nursing strategies involving in the daily life of senile dementia are discussed in this paper. The life fualify of the patient was emphasized as important as the severity, progression and symptoms of the illness, capacities of cognition and recognition, behavioral impairment and the daily activities. The most propriate pattern of taking care for the dementia patient was proposed as follows: for those inpatients and their family members, health education should properly be carried out, making use of varied modalities of vedio-audio means such as 
example, picture, record, cassetle and propaganda colume, so as to make the patient grasp abundant and systemic knowledge of health during their hospitalization; the detail measures should be directed against those patients living together, such as encouraging them to take exercises and participate in social activities as much as possible, intensifing the training of memory, counting capacities, manual operating and language communication; for the patients living in their own houses, it is important to enhance the early adaptability of family members in psychology and in active nursing.

127

The Psychotherapy Problems about Depression in General Hospital

Q.J. Shi. Department of Neurology and Psychiatry, Tongji Hospital, Tongji Medical College, Huazhong University of Science and Technology, Wuhan, 430030 P.R. China

In general hospital there are many patients who suffer from depression and go to see doctors because of their somatic symptoms. Many chronic patients or the patients who suffer from the malignant tumors develop the depressive status, too. Giving them anti-depressive drugs is not enough for treating those patients thoroughly. To understand them in psychodynamic way is quite useful for helping patients in a dimensional model and thus gets the better effects than those with only biological way. It leads us to understand our patients in a deeper level. Outer symptoms may represent inner conflicts; we can consider the inner psychotic world as the part of cause of the disease, differentiate the defense mechanisms as the fore-condition of the treatment, consider the patient-doctor relationship as the positive part of therapy, use confrontation, clarification and interpretation and take the family and social factors in account.

128

Depression symptom after acute stroke

M. Shu, H.J. Huang. Department of Neurology. Zhong Nan Hospital, Wuhan
University, Wuhan 430071, P.R. China

To explore the relationship between depression and the lesion location after stroke, the degree of nerve function lesions. 85 acute stroke patients were examined with Zung Depression Scale. The incidence of the depression symptom was $38.8 \%$ in acute stroke patients. There was a osculatory correlation between the depression and the lesion location of stroke; there was a positive correlation between the depression symptom and the degree of nerve function lesions.

129

Scheme in prevention and therapy of neuropathic disease in the aged H.Y. Zhao ${ }^{1}$, C.Z. Yan ${ }^{2}$, H. Lu ${ }^{3}$. ${ }^{1}$ Chinese Medical Association Wuhan Branch; ${ }^{2}$ Zhongnan hospital, Wuhan University; ${ }^{3}$ Laboratory of Wuhan Hospital Administration, P.R. China

With prolonged life span, Alzheimer disease, Parkinson disease and melancholia are becoming the most common diseases threatening the health of the elderly. This article put forward a scheme of developing social co-operative prevention and cure, placing emphasis on individualized treatment (using one's own brain for therapy), developing therapeutics vaccine and preparing the drugs which the conformation of the biochemistry of brain suits the conformation of patient's gene. Because the predominant lesion spots of these three diseases are in the cerebrum, so utilizing brain therapeutics is the most important method of prevention and cure at present and in the future. The medium involves: food which is beneficial to brain, music regulation, "poem-drugs" and "cognition" treatment, carrying out transplantation therapy, investigating psycho-pharmaco method and brain control equipment. With the 4th medicine revolution, the Nanometer Technique being extensive application in clinical medicine, we believe that treatment of these three diseases of brain will unceasingly gain the new breakthrough. 


\section{AUTHOR INDEX}

\begin{tabular}{|c|c|c|c|c|}
\hline Cao Alonso A. 1 & Huang H.J. 128 & Luo W.F. 112 & Tong J.N. 38 & Yang G.Y. 68 \\
\hline An W.L. 15 & Huang L.A. 71 & Ma J. 43 & Torres H.A.. 82,83 & Yang Q.D. 27, 96 \\
\hline Backman B.A. 84 & Huang R.X. 102 & Ma Y.X. 97, 99 & Tsujio I. 1 & Yang S.Y. 11,18 \\
\hline BAO S.Y. 112 & Huang S.S. 71 & Mandelkow E 6, 7 & Van Swieten J.C. 4 & Yang W.M. 76 \\
\hline Barghor S. N 6 & Hui Z.C. 122 & Mandelkow E. M. 6, 7 & Vogel R. 7 & Yang W.P. 42 \\
\hline Bergen M.V. 6 & Hyslop P.G.3 & Masters C. 33 & Wang J. Z 1102026 & Yang Y. 25, 26 \\
\hline Bi S. 87 & Jelic V. 100 & Mehta P. 18 & 4546497574117 & YAO Z.B. 38 \\
\hline Biernat J. 6 & Ji.Z.J. 29, 30, 44 & Meng Y.30 & Wang Z.F. 49, 46 & Yin M. 61 \\
\hline Bornstein N.M. 86 & Ji Q.M. 77 & Merz G. 11, 18 & Wang C.S. 93 & Ying L. 91 \\
\hline Brown T.C. 123 & Jiang J.P. 73 & Min B.Q. 107 & Wang D.Y. 41 & Yu J. 46 \\
\hline Cai Z. 32 & Jiang Y.B. 71 & Moessler H. 66 & Wang G.J. 114 & Yu S.Z. 115 \\
\hline Cao L.G. 61 & Jin C.F. 24 & Murayama O. 14 & Wang H.X. 84, 85 & Yu G. 3 \\
\hline X. B. 60 & Jue Q.M. 98 & Nie G.J. 24 & Wang J.G. 50 & Yu J. 46 \\
\hline Cao Y.L. 24 & Kamphorst W. 4 & Nie Z.Y. 91, 93, 116 & Wang L.J. 119 & Yu S.Z. 115 \\
\hline Castellani R.J. 8 & Kang Q.H. 31 & Nishimura M. 3 & Wang L.N. 27 & Yu X.F. 99 \\
\hline Chapman J. 86 & Karp A. 85 & Nordberg A. 5, 100 & Wang N.S. 86 & Yu X.F. 99 \\
\hline Chauhan A 9, 110 & Kasper S. 66 & Palmer K.84 & Wang Q. 45547574 & Yuan C.X. 56 \\
\hline Chauhan V. 110 & Khalil Z. 33 & Pan T.H. 72 & Wang Q.S. 59 & Zao X.C. 57 \\
\hline Chauhan Ved. S 9 & Khatoon S. 1 & Pei J.J. 1, 10 & Wang R. 29, 44 & Zeng K.B. 105 \\
\hline Chen A.B. 89 & Kim D.S 13 & Pei J. 15 & Wang T. 60 & Zeng X.Y. 73 \\
\hline Chen F.S. 3 & Korczyn A.D. 86 & Perry G. 8 & Wang T.H. 102 & Zhang M. 2328 \\
\hline Chen H.H. 34, 35 & Lai L.P. 103 & Qian C.Y. 55 & Wang X.C. 474954 & Zhang S. M. 23, 28 \\
\hline Chen J.P. 52 & Lee. M. H. 64 & Qiao Y.L. 91, 92, 116 & 7525 & Zhang B.L. 27 \\
\hline Chen K.N..36 & Li C.Y. 122 & Qin B. 73 & Wang X.D. 73, 111 & Zhang C.D. 41 \\
\hline Chen S.D. 21, 39, 40, 68 & Li F. 115 & Qin S. 59 & Wang X.F. 105 & Zhang G.R. 61 \\
\hline Chen W. 95 & Li T. 122 & Qin Y.58 & Wang X.R. 101 & Zhang J. 72 \\
\hline Chen X.W. 21, 68 & Li X.T. 54 & R.C. Green 123 & Wang Y.95 & Zhang J.G. 42 \\
\hline Chen X.Y. 125 & Li C.B. 89 & Ravid R. 4, 109 & Wang Y.D. 102 & Zhang J.H. 106 \\
\hline Chen Z. 101 & Li C.Y. 122 & Relkin N. 123 & Wang Y.P. 4546 & Zhang J.J. 62 \\
\hline Chen Z.B. 124 & Li F. 115 & Ren H.W. 31 & Wang Y.Z. 62 & Zhang J.L. 42 \\
\hline Cheng H..113 & Li G.Y. 118 & Ren H.Y. 106 & Wang Z.F. 4946 & Zhang J.Y. 44 \\
\hline Cui X. 22, 27 & Li H.G. 90 & Rhee S.K. 13 & Wegiel J 9, 110 & Zhang L 95 \\
\hline Cui,GY. 101 & Li J. 103 & Robakis N.K. 12 & Wei D.G. 50 & Zhang L. $67,69,92,116$ \\
\hline Ding J.D. 76 & Li L. 67,69 & Ru B.G. 31 & Wei F. 22 & Zhang M. 23, 28 \\
\hline Ding X.S. 113 & Li M. 79 & Ruan Y.W. 38 & Wen G.Y. 11 & Zhang M.Y. 89 \\
\hline Dong H.Q. 107 & Li P. 81, 103, 104 & Ruther E. 66 & Wen L.D.121 & Zhang R.Y. 69 \\
\hline Dong W.W. 118 & Li P.Y. 94 & Schneider A. 6 & Wen S.Q. 105 & Zhang S. M. 23, 28 \\
\hline Du X.P. 79 & Li Q.X. 33 & Schneider A. 6 & Wen X.C. 57 & Zhang T.T. 58 \\
\hline Fan J.H. 92 & Li T. 122 & Sengupta A. 1 & Weng Z.F.40 & Zhang X. 5, 22 \\
\hline Fan W.h. 36 & Li W. 94 & Shao M. 39, 40, 67 & Whitehouse P.J. 123 & Zhang X.J. 37 \\
\hline Fang Y.Y. 55 & Li W.B.22, 27 & Sheng S.L. 29, 32, 44 & Winblad B. $82,83,85$ & Zhang X.L. 113 \\
\hline Fang Z.Y. 25, 26 & Li X.T. 54 & Shi Q.J. 127 & Wouda J. 4 & Zhang X.Q. 107 \\
\hline Fei J. 72 & Li Z.G. 76 & Shi T.H. 108 & Wu J. 87 & Zhang Y. 87, 120 \\
\hline Feng H. 101 & Liang X.L. 119 & Shu F. 70 & Wu C.S. 92, 93 & Zhang Y.C. 46 \\
\hline Fratiglioni L. 82, 83, & Liao X.M. 20 & Shu M. 120,128 & Wu C.Y. 50 & Zhang Z.X. 98 \\
\hline 84,85 & Lin Z. 91 & Shu X.Y. 14 & Wu G.C. 101 & Zhao B.L. 24 \\
\hline Fu Z. 52 & Liu C.Q. 35 & Smith M.A. 8 & Wu J. 120 & Zhao J.H. 81 \\
\hline Gao J. 58 & Liu R. 47 & Stamer K. 7 & Wu J.C. 112 & Zhen Z.C. 21 \\
\hline Gong C. X. 1, 10, 15, 19 & Liu S.J 25 & Strauss E V. 83 & Wu Q.P. 94 & Zheng H.Y. 81 \\
\hline Grundke-Iqbal I. 1, 10, & Liu A.Q. 34, 35 & Sun X. Y. 2328 & Wu S.Q. 94 & Zheng k. 108 \\
\hline $15,19,64,75$ & Liu C.Q. 34, 35 & Sun.X.D. 116 & Wu Y.H. 51 & Zheng Z.P. 119 \\
\hline Guan Z.Z. 5 & Liu C.Y. 126 & Sun G.J. 43,80 & Xiang Y. 79 & Zhou K. 115 \\
\hline Guo M.L. 61 & Liu F.19, 67, 104 & Sun L. 75 & Хiao H. 95 & Zhou D.F. 93 \\
\hline Han J.X. 81 & Liu J. 68 & Sun S.G. $34,35,60,90$ & Xie L.OP. 42 & Zhou J.N. 59 \\
\hline Han M.X. 76 & Liu R. 47 & Sun X. Y. 23, 28 & Xie L.P. 77 & Zhou J.Z. 103 \\
\hline Haque N. 1 & Liu S.J. 25, 26 & Takashima A. 14, 65 & Xu B. 52 & Zhou K. 115 \\
\hline Harris P.L.R. 8 & Liu X.G. 45,47 & Takeda M. 2 & Xu D.H. 78 & Zhou Y.H. 96 \\
\hline He C.Z. 23, 28 & Liu X.H. 88 & Tan P.Z. 96 & Xu H.X. 16 & Zhu C. Q. 48, 53, 63 \\
\hline He S.S. 117 & Liu X.Q. 108 & Tan X.L. 118 & Xu J. 55 & Zhu H.F. 126 \\
\hline Не X.Y 11, 18 & Liu Y. 77 & Tang A.W. 119 & Xu R.S. 51 & Zhu L. 78 \\
\hline He Y.L. 89 & Liu Y.L. 37 & Tang H.D. 40 & Xue D.L. 70 & Zhu Y. 97 \\
\hline Hong Z. 98 & Liu Z.G. 21, 39, 68 & Tang M.N. 88 & Xue S.R. 33 & Zou X.Y. 88 \\
\hline Hong D.J. 53 & Liu Z.P. 72 & Tang X.L. 96 & Yamaguchu H. 14, 17 & \\
\hline Hong H. 102 & Long Y.G. 5 & Tatebayashi Y. 64 & Yan Y. 105 & \\
\hline Hu J.M. 70 & Lu P.F. 99, 97 & Tian H.B. 77 & Yang W.P. 42 & \\
\hline Hu Y.Y. 117 & Lu S.D. 53 & Tian Ts. 43, 80 & Yang Y. 2526 & \\
\hline Huang F.N. 42 & Lu S.Y. 58 & Tong Et. 34,51 & Yang F. 29 & \\
\hline
\end{tabular}

